\title{
Synthesis, Characterization and Reactivity of Neutral Octahedral Alkyl-Cobalt(III) Complexes Bearing a Dianionic Pendadentate Ligand.
}

\author{
Lucie Nurdin, Warren E. Piers, ${ }^{*}$ Jian-Bin Lin and Benjamin S. Gelfand \\ Department of Chemistry, University of Calgary, 2500 University Dr. NW, Calgary, AB, \\ Canada T2N 1N4
}

\begin{abstract}
A variety of neutral alkyl-cobalt(III) complexes bearing a dianionic tetrapodal pendadentate ligand $\mathrm{B}_{2} \mathrm{Pz} 4 \mathrm{Py}$ are reported. Compounds 2-R ( $\mathrm{R}=\mathrm{CH}_{3}, \mathrm{CH}_{2} \mathrm{SiMe}_{3}$, $\mathrm{CH}_{2} \mathrm{SiMe}_{2} \mathrm{Ph},{ }^{i} \mathrm{Bu}, \mathrm{CH}_{2}\left(\mathrm{c}-\mathrm{C}_{5} \mathrm{H}_{9}\right)$ and $\left.\left(\mathrm{CH}_{2}\right)_{4} \mathrm{CH}=\mathrm{CH}_{2}\right)$ are synthesized in $58-90 \%$ yield. These diamagnetic, octahedral complexes are thermally stable up to $110^{\circ} \mathrm{C}$ and are also remarkably stable to ambient atmosphere. They were fully characterized by spectroscopic techniques, and in three cases, X-ray crystallography. Evidence for reversible homolytic cleavage of the Co-C bonds was found in their reactions with the hydrogen atom donor 1,4cyclohexadiene and the radical trap TEMPO, as well as the observed cyclization of the 5hexenyl group to the methylcyclopentyl derivative over the course of several hours. Despite these observations, it can be concluded that the diborate $\mathrm{B}_{2} \mathrm{Pz}_{4} \mathrm{Py}$ ligand provides a very stable platform for these Co(III) alkyls. Reduction by one electron to a Co(II) alkyl can accelerate bond homolysis, but in this instance, using cobaltocene as the reducing agent, leads to ejection of an alkide anion through bond heterolysis, an unusual reaction for Co(III) alkyls. Finally, protonation of compound 2-Me with the strong acid $\mathrm{HNTf}_{2}$ leads to divergent reactivity in which the major protonation site is the pyridyl nitrogen of the ligand as opposed to protonation of the methyl group. The produce of protonation at nitrogen is the dimeric species 4 which was prepared via separate synthesis and characterized by Xray crystallography.
\end{abstract}

\section{Introduction.}

In Nature, two types of cobalamins, also known as Vitamin B12, exhibit a Co-C bond, methylcobalamin (MeCbl) ${ }^{1}$ and adenosylcobalamin (AdoCbl), ${ }^{2}$ featuring $-\mathrm{CH}_{3}$ and $-\mathrm{CH}_{2} \mathrm{R}$ groups in the axial position, respectively (Figure 1). In both stable forms of Vitamin B12, the cobalt center bears a +III oxidation state. During catalysis, however, the Co-C bond of $\mathrm{MeCbl}$, can undergo heterolytic cleavage to give $\mathrm{Co}(\mathrm{I})$ and $\mathrm{CH}_{3}{ }^{+}$, while for AdoCbl the bond can cleave homolytically to provide a source of Co(II) and organic radical. ${ }^{3}$ Thus, the alkyl groups in these Co(III) alkyls exhibit divergent reactivity in the in vivo function of Vitamin B12. Although both $\mathrm{MeCbl}$ and AdoCbl bear the same functionalized corrin $\mathrm{L}_{3} \mathrm{X}$ supporting 
ligand, the side chain terminating in a benzimidazole function which serves as the second axial ligand ${ }^{4}$ can dissociate or bind during the catalytic transformations mediated by Vitamin B12, modulating the behavior of the Co-C bond. ${ }^{5}$

Since the discovery of the cobalamin coenzymes, many synthetic alkyl-cobalt(III) complexes have been reported in an effort to mimic and model the properties of Vitamin B12. A recent review by Detrembleur et al. codifies the main categories of reported synthetic alkyl-cobalt(III) complexes, sorting them according to the nature of the supporting ligand. ${ }^{6}$ While the bulk of the compounds reported enlist porphyrin, cobaloxime, Schiff base or bis-(acetylacetonate) multidentate supporting ligands, other systems are potentially of interest. For example, tetrapodal pentadentate ligands, ${ }^{7,8}$ can provide a well-defined platform around the metal center that mimics the five coordinate
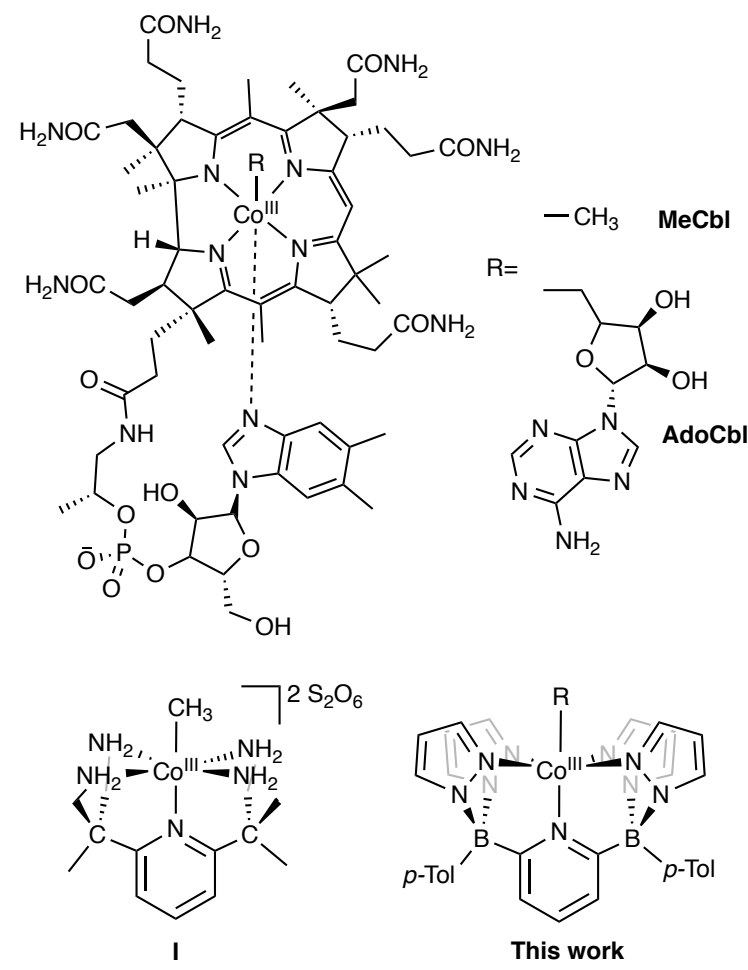

Figure 1. Schematic representation of alkyl-Co(III) complexes.

environment of the B12 cobalamin construct. ${ }^{9}$ Such ligands have been deployed across the transition series and used for a variety of small molecule activation and catalytic transformations such as the reduction of water to hydrogen ${ }^{10-13}$ or the oxidation of water 
to oxygen. ${ }^{14,15}$ Despite the extensive chemistry of such ligands, only a single example of a Co(III) alkyl species supported by a pentadentate ligand has been structurally characterized, the $\mathrm{pyN}_{4}$ cobalt methyl complex I of Grohmann et al. shown in Figure 1.16 However, its reactivity was not investigated, and the effect of the rigidity imposed by the pentadentate platform around the cobalt center remains unclear.

Based on the well-known pentapyridyl ( $\mathrm{PY}_{5}$ ) system introduced 20 years ago, 17, 18 and later on the work by Gardinier who substituted the equatorial pyridine arms with pyrazole donors, ${ }^{19}$ our group has reported the synthesis of a $\mathrm{B}_{2} \mathrm{Pz}_{4} \mathrm{Py}$ ligand which features two borate moieties. ${ }^{20-22}$ The introduction of the borate linkers renders the ligand dianionic and therefore more electron-rich. This stabilizes higher oxidation state intermediates so they can be studied in more detail. For $\mathrm{Co}$, the $\mathrm{B}_{2} \mathrm{Pz}_{4} \mathrm{Py}$ ligand moves the $\mathrm{Co}(\mathrm{II}) / \mathrm{Co}(\mathrm{III})$ couple more than $0.5 \mathrm{~V}$ to more positive potentials, suggesting that it is a suitable platform for octahedral Co(III) alkyls. Herein we report the synthesis and properties of a family of neutral Co(III) alkyl complexes of the general formula shown in Figure 1.

\section{Results and Discussion.}

\section{Synthesis and Characterization of 2-R}

The Co(III) bromide complex $\mathbf{1}^{22}$ is a convenient starting material for a family of Co(III) alkyls (Scheme 1). Treatment of $\mathbf{1}$ in toluene with the corresponding alkyllithium reagent
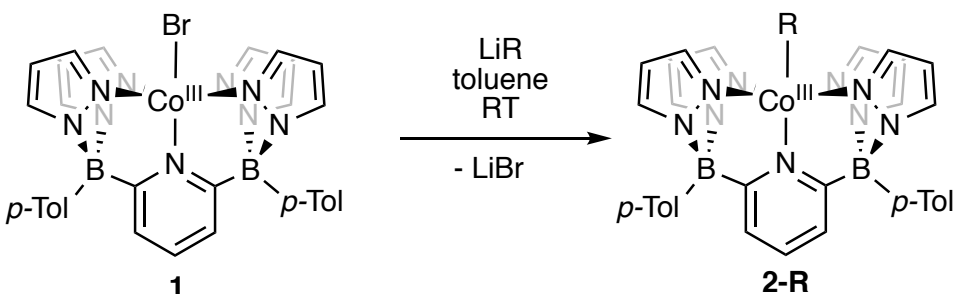

\begin{tabular}{|lllccc|}
\hline $\mathbf{R}$ & Yield & Cmpd & $\begin{array}{c}\delta \mathbf{C} \\
(\mathrm{ppm})\end{array}$ & $\begin{array}{c}\delta \mathrm{H} \\
\left(\mathbf{1} \mathbf{J}_{\mathrm{CH}}\right.\end{array}$ \\
\hline $\mathrm{CH}_{3}$ & $70 \%$ & 2-Me & 19.1 & 4.13 & 135 \\
$\mathrm{CH}_{2} \mathrm{SiMe}_{3}$ & $\mathbf{8 8 \%}$ & 2- $\mathrm{CH}_{2} \mathrm{SiMe}_{3}$ & 16.6 & 4.10 & 119 \\
$\mathrm{CH}_{2} \mathrm{SiMe}_{2} \mathrm{Ph}$ & $71 \%$ & 2- $\mathrm{CH}_{2} \mathrm{SiMe}_{2} \mathrm{Ph}$ & 14.3 & 4.27 & 120 \\
$\mathrm{CH}_{2} \mathrm{CH}\left(\mathrm{CH}_{3}\right)_{2}$ & $58 \%$ & 2-' $\mathrm{Bu}$ & 43.7 & 4.88 & 133 \\
\hline
\end{tabular}

Scheme 1. Synthesis of $\mathbf{2}-\mathbf{R}$ by salt metathesis 
in a 1:1 ratio affords the alkyl-Co(III) complexes 2-R (Scheme 1) in moderate to excellent yield. Various alkyl groups can be introduced, ranging from methyl (2-Me), to bulker groups (2- $\mathrm{CH}_{2} \mathrm{SiMe}_{3}$ and $2-\mathrm{CH}_{2} \mathrm{SiMe}_{2} \mathrm{Ph}$ ) and an alkyl group with a beta-hydrogen (2-i $\mathrm{Bu}$ ). The reaction proceeds quickly at room temperature, as observed by a color change from dark orange (1) to light pink (2-R), accompanied by the formation of a white precipitate (LiBr). The products are all diamagnetic and fully characterizable by NMR spectroscopy (vide infra). Although these reactions proceed cleanly without reduction of $\mathbf{1}$, the organometallic products are prone to reduction to Co(II) by excess RLi. Additional equivalents of RLi resulted in the disappearance of the diamagnetic peaks in the ${ }^{1} \mathrm{H}$ NMR spectrum and emergence of the distinct signal at $+75 \mathrm{ppm}$ in the ${ }^{11} \mathrm{~B}$ NMR spectrum, attributed to the previously reported Co(II) complex. ${ }^{22}$ Therefore, a strict 1:1 ratio of $\mathbf{1}$ and alkyllithium was used for the syntheses of compounds 2-R.

The low spin, $\mathrm{d}^{6}$ octahedral alkyl-cobalt(III) complexes $\mathbf{2}-\mathbf{R}$ are stable at room temperature under ambient atmosphere for a few hours, which is surprising since many alkyl-cobalt(III) complexes have been shown to react with oxygen to form alkylperoxo complexes. ${ }^{23,}{ }^{24}$ In addition, 2-R are thermally stable (tested up to $110{ }^{\circ} \mathrm{C}$ in toluene). They were fully characterized using multi-nuclei NMR spectroscopy, mass-spectrometry and elemental analysis, and all the characterization data can be found in the experimental section. Through spectral analysis of the 2D NMR spectra of 2-R, the peaks ranging from 4.13 (2Me) to $4.10\left(2-\mathbf{C H}_{2} \mathbf{S i M e}_{3}\right), 4.27\left(2-\mathbf{C H}_{2} \mathbf{S i M e}{ }_{2} \mathbf{P h}\right)$ and $4.88\left(2-{ }^{-} \mathbf{B u}\right)$ were assigned to the protons located on the $\alpha$ carbon of the ligating alkyl group (Table inset, Scheme 1), which are close to the range of chemical shift of reported peaks of other alkyl-Co(III) complexes. ${ }^{16}$, ${ }^{25}$ The ${ }^{1} \mathrm{~J}_{\mathrm{CH}}$ coupling constants for the $\alpha \mathrm{C}-\mathrm{H}$ bonds are in good agreement with the values reported for the free alkanes, ${ }^{26}$ consistent with classical, non-agostic structures for these compounds. In addition, three of the 2-R derivatives were characterized using X-ray crystallography (Figure 2). From the structures, the complexes exhibit an overall octahedral geometry with a Co-C bond length similar to other alkyl-Co(III) complexes. ${ }^{16,25}$, 27, 28 In particular, the metrical data of 2-Me is comparable to that of MeCbl with a Co-C bond length of 1.987 (4) vs 1.99 for MeCbl and Co-N1 of 2.072 (3) vs 2.19 in MeCbl. ${ }^{29}$ Notably, the axial Co-N1 bond of 2-Me is shorter than the Co- $\mathrm{N}_{\mathrm{py}}$ bond using the $\mathrm{pyN}_{4}$ 

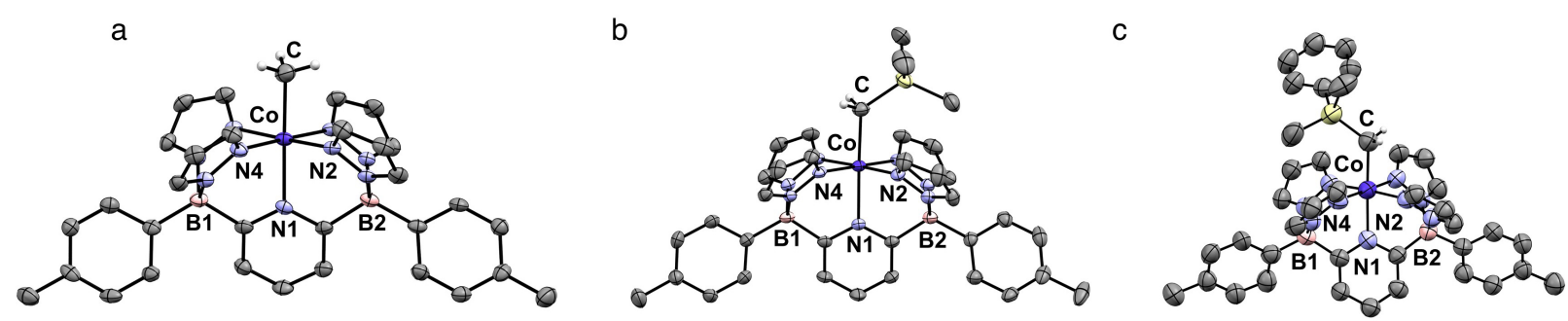

Figure 2. Thermal ellipsoid diagrams for 2-Me (a), 2- $\mathbf{C H}_{2} \mathrm{SiMe}_{3}$ (b) and $2-\mathrm{CH}_{2} \mathrm{SiMe}_{2} \mathrm{Ph}$ (c). Hydrogen, boron, carbon, nitrogen, silicon and cobalt atoms are white, pink, grey, light blue, light yellow and dark blue respectively. Thermal ellipsoids are shown at the 50\% probability level. Calculated hydrogen atoms are omitted for clarity except the ones of the alpha carbon. Solvent molecules are omitted for clarity. Selected bond distances ( $)$ and bond angles ( ${ }^{\circ}$ ): a. Co-C, 1.987(4); Co-N1, 2.072(3); Co-N2, 1.914(3); Co-N4, 1.927(3); N1Co-C, 178.3(1); N1-Co1-N2, 90.6(1). b. Co-C, 2.017(3); Co-N1, 2.083(2); Co-N2, 1.957(4); Co-N4, 1.988(3); N1-Co-C, 177.8(2); N1-Co1-N2, 90.0(1). c. Co-C, 2.03(1); Co-N1, 2.098(8); Co-N2, 1.920(8); Co-N4, 1.978(7); N1-Co-C, 175.6(4); N1-Co1-N2, 88.1(3). Selected metrical data given in Table S1.

platform (2.018(2)), showing how the pyrazole arms impose a more rigid environment around the cobalt center in comparison to alkylamines. The Co-N1 bond length in 2-Me is slightly elongated in comparison to the Co-N1 bond of 1 (2.005 (2)). This is attributed to the stronger trans influence of the alkyl group vs. the bromo ligand. ${ }^{30}$ As in other structures involving this ligand environment, ${ }^{20-22,31,32}$ the bulkier silylalkyl groups orient themselves along the vector bisecting the narrower N-Co-N angle, which is more sterically open than the perpendicular vector bisecting the wider N-Co-N angles. Nevertheless, rotation about the Co-C bonds is rapid on the NMR timescale, averaging the environments of the four pyrazolyl rings at all accessible temperatures.

\section{Properties of the Co-C bonds in 2-R}

The relatively electron richness of the dianionic $\mathrm{B}_{2} \mathrm{Pzz}_{4} \mathrm{Py}$ ligand environment contributes to the apparent stability of these octahedral Co(III) alkyl complexes. Co(III)-C bonds tend to be fairly weak (14-40 $\mathrm{kcal} \mathrm{mol}^{-1}$ ) and subject to thermal homolysis via $\mathrm{k}_{1}$ (Scheme 2).6,33-36 Typically, the equilibrium lies towards the alkyl and k-1 is essentially diffusion controlled 
such that the $\mathrm{R} \cdot$ radical only slowly escapes the solvent cage; the presence of a radical trapping agent can accelerate this process by drawing the equilibrium to the right. Bond homolysis may also be accelerated by one electron reduction (via $\mathrm{k}^{\mathrm{R} \cdot}$ ) since addition of an electron populates an antibonding orbital and lowers the Co-C bond order. However, in situations where Co(I) is less accessible, loss of $\mathrm{R}^{-}$via $\mathrm{k}^{\mathrm{R}-}$ (Scheme 2 ) becomes a possibility although this has not been directly observed to our knowledge. Therefore, given the historical importance of such compounds as models for the behavior of cobalamin alkyls, it is of interest to probe the reactivity of these species towards radical trapping agents, oneelectron reducing agents and protons.

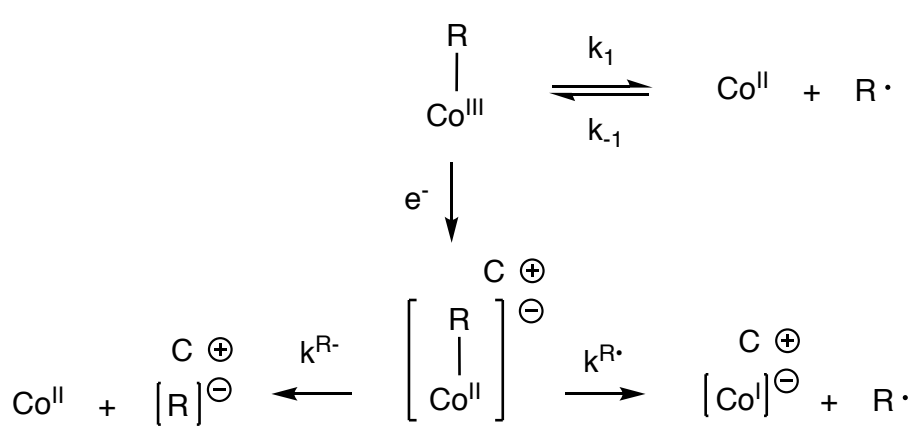

Scheme 2. Pathways for cleavage of the Co-C bond in alkyl-Co(III) complexes

The Co-C bond dissociation energies (BDE) for 2-R were evaluated using DFT calculations with the B3PW91 functional (for details, see the Supporting Information) and result in values of 32.1 (2-Me, kcal mol-1), $29.3\left(2-\mathbf{C H}_{2} \mathbf{S i M e}_{3}\right), 31.6\left(2-\mathrm{CH}_{2} \mathrm{SiMe}_{2} \mathrm{Ph}\right)$ and 27.6 (2iBu) for the four synthesized compounds. These are consistent with experimental and computed values ${ }^{6}$ for other six coordinate Co(III)-C complexes (perhaps towards the high end of the range) but follow a general trend of weaker bonds for sterically more imposing $\mathrm{R}$ groups. ${ }^{33}$

Based on these values, it might be expected that the homolytic bond cleavage equilibrium in Scheme 2 can be apprehended using radical trapping agents such as 1,4-cyclohexadiene (CHD) or 2,2,6,6-Tetramethylpiperidin-1-yl)oxyl (TEMPO), Scheme 3. While compounds 2$\mathbf{R}$ are stable towards bond homolysis up to $110^{\circ} \mathrm{C}$, in the presence of the hydrogen atom donor $\mathrm{CHD},(\mathrm{BDE}=71 \mathrm{kcal} / \mathrm{mol}),{ }^{37}$ all of the compounds except 2-Me slowly released R-H 

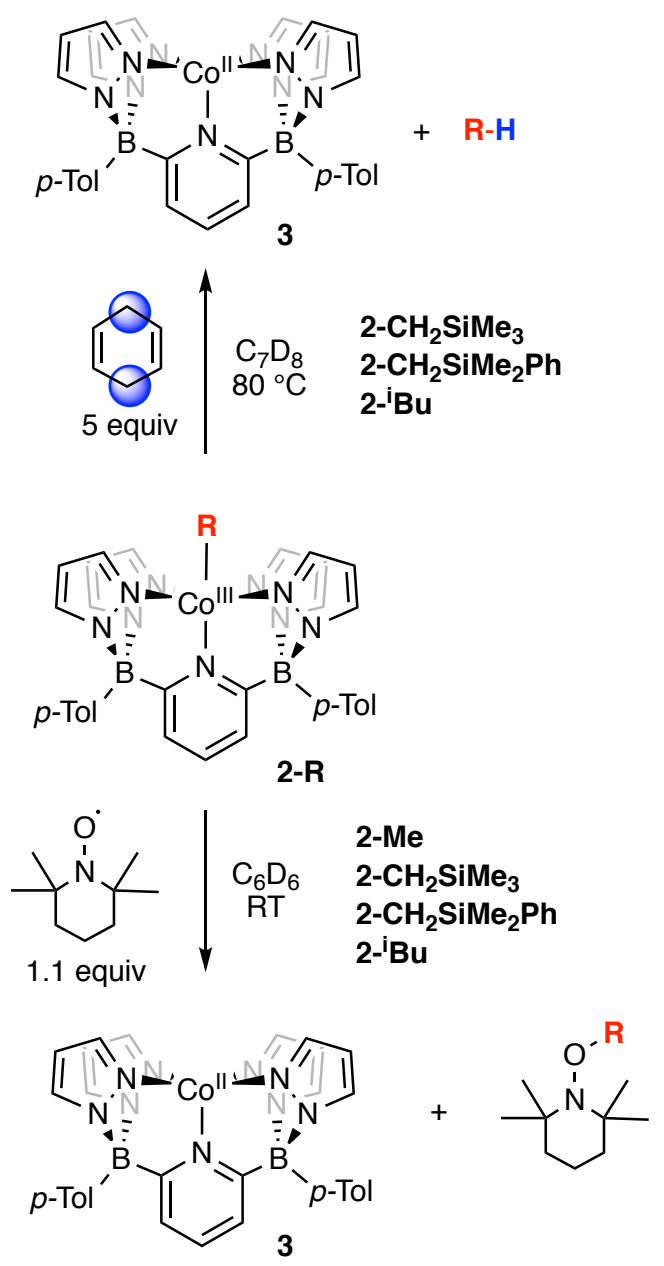

Scheme 3. Reactivity of 2-R with R・ trapping agents.

at $80^{\circ} \mathrm{C}$, along with the known five coordinate $\left(\mathrm{B}_{2} \mathrm{Pz}_{4} \mathrm{Py}\right) \mathrm{Co}(\mathrm{II})$ complex 3 , identifiable via its characteristic ${ }^{11} \mathrm{~B}$ NMR chemical shift of 75 ppm (Figures S1-S7). ${ }^{21}$ Protio benzene was also detected in these reactions. This suggests that under these conditions, the R• formed via bond homolyis can be trapped when it is sterically large enough to escape the solvent cage; in the case of 2-Me, this does not occur. More efficient trapping of R• from all of the cobalt (III) alkyls is seen when TEMPO is employed (Scheme 3, bottom). Here, the reactions occur smoothly at room temperature over $\approx 24 \mathrm{hrs}$ to produce 3 and the TEMPO-R products in excellent NMR yields (Figures S8-S15). In these TEMPO trapping reactions, once bond cleavage and cage escape occurs, reaction of R $\bullet$ with TEMPO is essentially diffusion controlled, whereas when CHD is the trapping agent, there is an added barrier involving $\mathrm{H}$ atom transfer, accounting for the harsher conditions necessary to effect those reactions. 
Both outcomes are consistent with a homolytic cleavage pathway as depicted in Scheme 2. Qualitatively, in comparison to other literature accounts, the thermal stability of 2-R both in the absence and presence of $\mathrm{R} \bullet$ trapping agents indicate they are generally more robust towards thermal homolytic bond cleavage than most six coordinate Co(III) alkyl compounds.

Further evidence for the rapid and reversible cleavage of 2-R was obtained by preparing alkyl derivative $\mathbf{2 - (}\left(\mathbf{C H}_{2}\right)_{4} \mathbf{C H}=\mathbf{C H}_{2}$, featuring the "radical clock" 5-hexenyl ${ }^{38}$ group. If homolytic cleavage is occurring, conversion of this species to $\mathbf{2}-\mathbf{C H}_{2}\left(\boldsymbol{c}-\mathbf{C}_{5} \mathbf{H}_{9}\right)$ should be observed. A summary of this experiment is shown in Scheme 4. The 5-hexenyl lithium

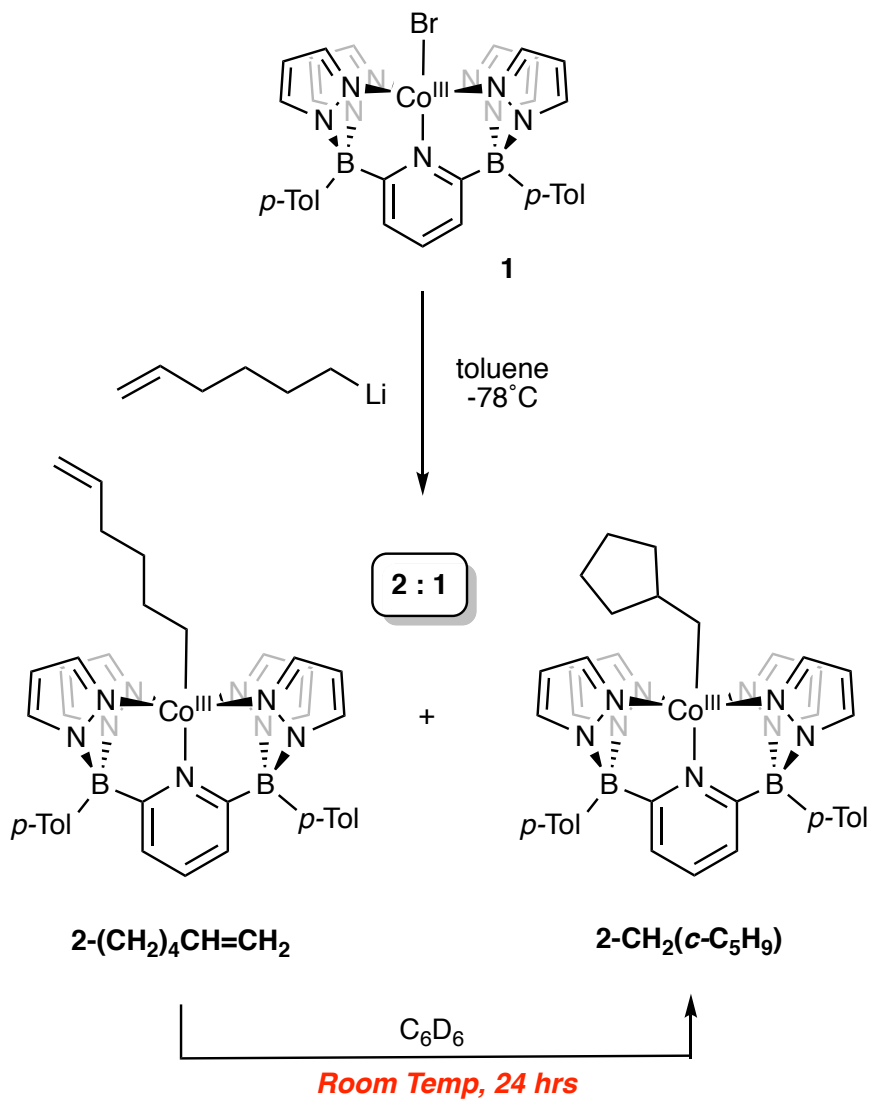

Scheme 4. Radical clock experiment.

reagent was generated in situ from the requisite iodo-alkane and tBuLi and added to a solution of $\mathbf{1}$ in toluene; it was not possible to prevent some cyclization to methylcyclopentyl lithium even at low temperature ${ }^{39}$ and so an 2:1 mixture of 2$\left(\mathbf{C H}_{2}\right)_{4} \mathbf{C H}=\mathbf{C H}_{2}$ and $\mathbf{2}-\mathbf{C H}_{2}\left(\boldsymbol{c}-\mathbf{C}_{5} \mathbf{H}_{9}\right)$ was produced in this synthesis (Figure S16). The 
identity of $\mathbf{2}-\mathbf{C H}_{2}\left(\boldsymbol{c}-\mathbf{C}_{5} \mathbf{H}_{9}\right)$ was confirmed through separate synthesis ( $90 \%$ isolated yield) from $\mathbf{1}$ and methylcyclopentyl lithium. The 2:1 mixture converted fully to $\mathbf{2}-\mathbf{C H}_{2}\left(\boldsymbol{c}-\mathbf{C}_{5} \mathbf{H}_{9}\right)$ after $24 \mathrm{~h}$ at room temperature in $\mathrm{C}_{6} \mathrm{D}_{6}$ we observed its full consumption and the formation of $\mathbf{2}-\mathbf{C H}_{2}\left(\boldsymbol{c}-\mathbf{C}_{5} \mathbf{H}_{9}\right)$ (Figures S17-18). The cyclization is rather slow and a small amount of $\mathbf{3}$ is observed to form by ${ }^{11} \mathrm{~B}$ NMR spectroscopy, suggesting that some R• escapes during this process. Nonetheless, the experiment indicates that reversible bond homolysis is occurring in this system.

The above observations suggest that for the $\mathrm{K}_{1}$ equilibrium of Scheme 2, the ratio of $\mathrm{k}_{1} / \mathrm{k}_{-1}$ is very low and that trapping of alkyl radicals by Co(II) complex $\mathbf{3}$ is rapid, contributing to the overall macroscopic stability of these alkyls. Martin and Finke have shown that one electron reduction of $\mathrm{MeCbl}$ results in significantly enhanced rates of bond homolyis. ${ }^{34}$ Indeed, cyclic voltammetric measurements on compounds 2-R indicate that all of them undergo an irreversible one-electron reduction at potentials of - $0.89 \mathrm{~V}$ (2-Me), -0.86 V (2$\left.\mathrm{CH}_{2} \mathrm{SiMe}_{3}\right), 00.88 \mathrm{~V}\left(2-\mathrm{CH}_{2} \mathrm{SiMe}_{2} \mathrm{Ph}\right)$ and $-0.85 \mathrm{~V}\left(2-{ }^{-} \mathrm{Bu}\right)$, all vs $\mathrm{Fc} / \mathrm{Fc}+$. Figure 3 shows the $\mathrm{CV}$ of 2-Me at various scan rates and the data fo the other $\mathbf{2 - R}$ compounds are shown in

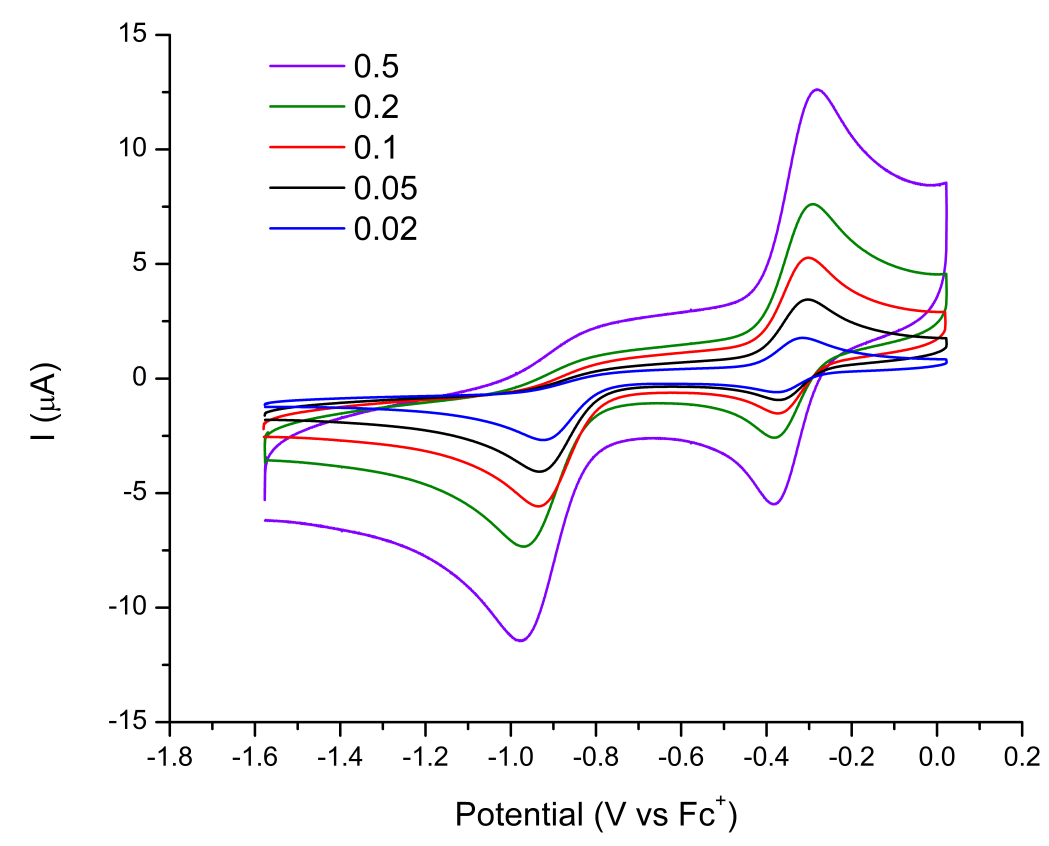

Figure 3. Cyclic voltammograms of 2-Me in THF at different scan rates $(0.02$ to $0.5 \mathrm{~V} / \mathrm{s})$ under argon. Supporting electrolyte: $\mathrm{TBAPF}_{6}$; working electrode: glassy carbon; counter electrode: Pt; reference electrode: Ag wire. 
Figures S19-21). This suggests that the addition of one electron to $2-\mathbf{R}$ leads to a highly unstable radical anion [Co(II)-R] ${ }^{\bullet}$. However, interpretation of the cyclic voltammogram suggests that this radical anion does not decompose via bond homolyis (via $\mathrm{k}^{\mathrm{R}}$, Scheme 2) but rather via dissociative loss of R- i.e. heterolytic cleavage of Co-C. As seen in Figure 3, for 2-Me, when scanned in the range of 0.1 to $-1.6 \mathrm{~V}$ at variable scan rates ( 0.02 to $\left.0.5 \mathrm{~V} \mathrm{~s}^{-1}\right)$, a reduction wave at $-0.89 \mathrm{~V}(\mathrm{vs} \mathrm{Fc} / \mathrm{Fc}+$ ) is observed to be partially reversible at faster scan rates, but is essentially irreversible at slower scan rates. Anodic scanning shows a pseudo reversible oxidation at $-0.33 \mathrm{~V}$, which is identical to the potential observed for the $\mathrm{Co}(\mathrm{II}) / \mathrm{Co}(\mathrm{III})$ couple of five coordinate complex 3 under the same conditions, ${ }^{22}$ suggesting that $\mathbf{3}$ is the product of decomposition of the one electron reduction product of $\mathbf{2}$-Me. If bond homolysis from [Co(II)-R]•- occurred via $\mathrm{k}^{\mathrm{R} \bullet}$, the product would be the $[\mathrm{Co}(\mathrm{I})]^{-}$anion, that is the one electron reduction product of $\mathbf{3}$. Electrochemical measurements on $\mathbf{3}$ itself show that the $\mathrm{Co}(\mathrm{I}) / \mathrm{Co}(\mathrm{II})$ couple in this compound occurs at very negative potentials $(\approx-$ $2.54 \mathrm{~V}$ vs $\mathrm{Fc} / \mathrm{Fc}+$, see Figure S22) so this clearly is not the product of decomposition of [Co(II)-R]“-, which prefers to eject alkyl anions R-

This notion was supported by experiments that probe the one electron chemical reduction of compounds 2-R. For those with larger alkyl groups than methyl, reduction with cobaltocene $\left(\mathrm{Cp}_{2} \mathrm{Co}\right)$ in benzene proceeds cleanly as depicted in Scheme 5 . These reactions occur essentially upon mixing and cleanly produce compound 3, along with the $\mathrm{CpCo}(\mathrm{I})$ cyclopentadiene complexes I-R (Figures S23-28). These are the known products of the reactions of the cobalticenium cation with bulky alkide nucleophiles; ${ }^{40}$ although these specific examples are new, we did not pursue isolation/characterization of these byproducts. They are formed in $>90 \%$ NMR spectroscopic yield and exhibit characteristic resonances in the ${ }^{1} \mathrm{H}$ NMR spectra for the allylic cyclopentadienyl hydrogen and the $\mathrm{CH}_{2}$ groups of the added alkyl group (shown in Scheme 5). Thus, it appears clear that the $\mathrm{Cp}_{2} \mathrm{Co}$ reduces $2-\mathbf{R}$, and the resulting radical anion ejects $R^{-}$that adds to the $\left[\mathrm{Cp}_{2} \mathrm{Co}\right]^{+}$as encountered in well-established chemistry.

Interestingly, despite having a similar reduction potential to the others, the same reaction with 2-Me appears to not to proceed; mixing 2-Me with $\mathrm{Cp}_{2} \mathrm{Co}$ results in no reaction at room temperature (Figure S29). We believe this stems from divergent reactivity of $\left[\mathrm{Cp}_{2} \mathrm{Co}\right]^{+}$with the methide anion in comparison to the bulkier alkide anions discussed 


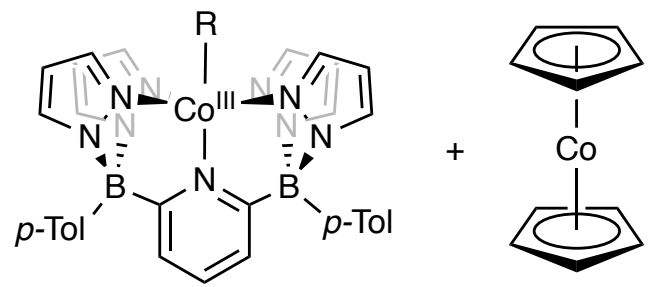

2-R

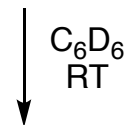

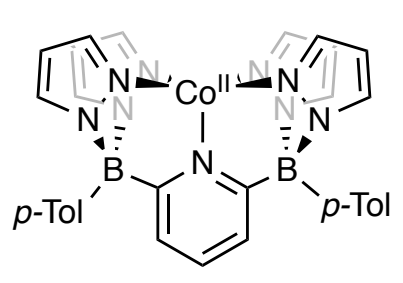

3

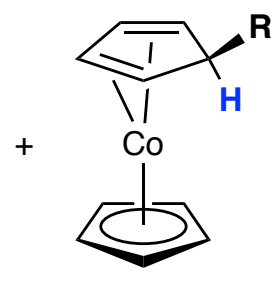

I-R

\begin{tabular}{lll}
\hline R & $\delta \mathrm{H}$ & $\delta \mathrm{H}_{2}$ \\
& \multicolumn{2}{c}{ (ppm) } \\
\hline $\mathrm{CH}_{2} \mathrm{SiMe}_{3}$ & 2.73 & 0.00 \\
$\mathrm{CH}_{2} \mathrm{SiMe}_{2} \mathrm{Ph}$ & 2.76 & 0.30 \\
$\mathrm{CH}_{2} \mathrm{CH}\left(\mathrm{CH}_{3}\right)_{2}$ & 2.67 & 0.48
\end{tabular}

Scheme 5. One electron reduction of $2-\mathbf{R}(\mathrm{R} \neq \mathrm{Me})$

above ${ }^{40}$ Rather than add to one of the $\mathrm{Cp}$ rings, Me- reduces the cobalticinium cation back to cobaltocene, and the methyl radical that results dimerizes to ethane or scavenges a hydrogen atom to produce methane. Indeed, we reproduced this chemistry and observed only a trace of I-Me upon reaction of $\left[\mathrm{Cp}_{2} \mathrm{Co}\right]^{+}\left[\mathrm{PF}_{6}\right]^{-}$with $\mathrm{MeLi}$, the major products being cobaltocene and ethane (Figures S30-31). In the case of reduction of 2-Me, the Me• produced is trapped rapidly by 3 , to regenerate $2-\mathbf{M e}$, giving the appearance of no reaction (Scheme 6). Upon heating the benzene solutions of 2-Me and $\mathrm{Cp}_{2} \mathrm{Co}$ to $80^{\circ} \mathrm{C}$ for 24 hours, traces of I-Me were observed, but otherwise the sample was unchanged (Figure S32).

The relative electron richness of these neutral Co(III) alkyls (in comparison to cationic $\mathrm{Co}(\mathrm{III})$ alkyls) lead us to also explore the reactivity of alkyls 2-Me and 2- $\mathbf{C H}_{2} \mathbf{S i M e}_{3}$ towards protic reagents. Both are relatively inert towards weaker acids like water and alcohols. The 
stronger acid bis(trifluoromethanesulfonyl)imide acid $\left(\mathrm{HNTf}_{2}\right)$ did react with the alkyls, although for the bulker compound the reaction was not clean, and indeed much of 2$\mathbf{C H}_{2} \mathbf{S i M e}_{3}$ remained unreacted (Figure S33). However, when 2-Me was treated with one equivalent of $\mathrm{HNTf}_{2}$, a purple precipitate was rapidly formed and a new ${ }^{1} \mathrm{H}$ NMR spectrum recorded (Figures S34-35). In the ${ }^{1} \mathrm{H}$ NMR spectrum both ethane and methane were observed according to the chemical shifts referenced against $\mathrm{C}_{6} \mathrm{D}_{6}$ (Figure S37). ${ }^{41}$ Based on the ${ }^{1} \mathrm{H}$ NMR integration of $\mathrm{C}_{2} \mathrm{H}_{6}$ vs $\mathrm{CH}_{4}$, a rough estimation of a 4:1 ratio of $\mathrm{C}_{2} \mathrm{H}_{6}: \mathrm{CH}_{4}$ was found (Figure S37). A small amount of the known diamagnetic compound $\left[\left(\mathrm{B}_{2} \mathrm{Pz}_{4} \mathrm{Py}\right) \mathrm{Co}(\mathrm{III})\right]^{+}\left[\mathrm{NTf}_{2}\right]^{-}$was identified by ${ }^{1} \mathrm{H}$ and ${ }^{19} \mathrm{~F}$ NMR spectroscopy, ${ }^{22}$ but the bulk of the cobalt containing product was the paramagnetic purple precipitate, which was identified to be the dimeric Co(II) complex 4 by X-ray crystallography (Figure 4, Scheme 7.)
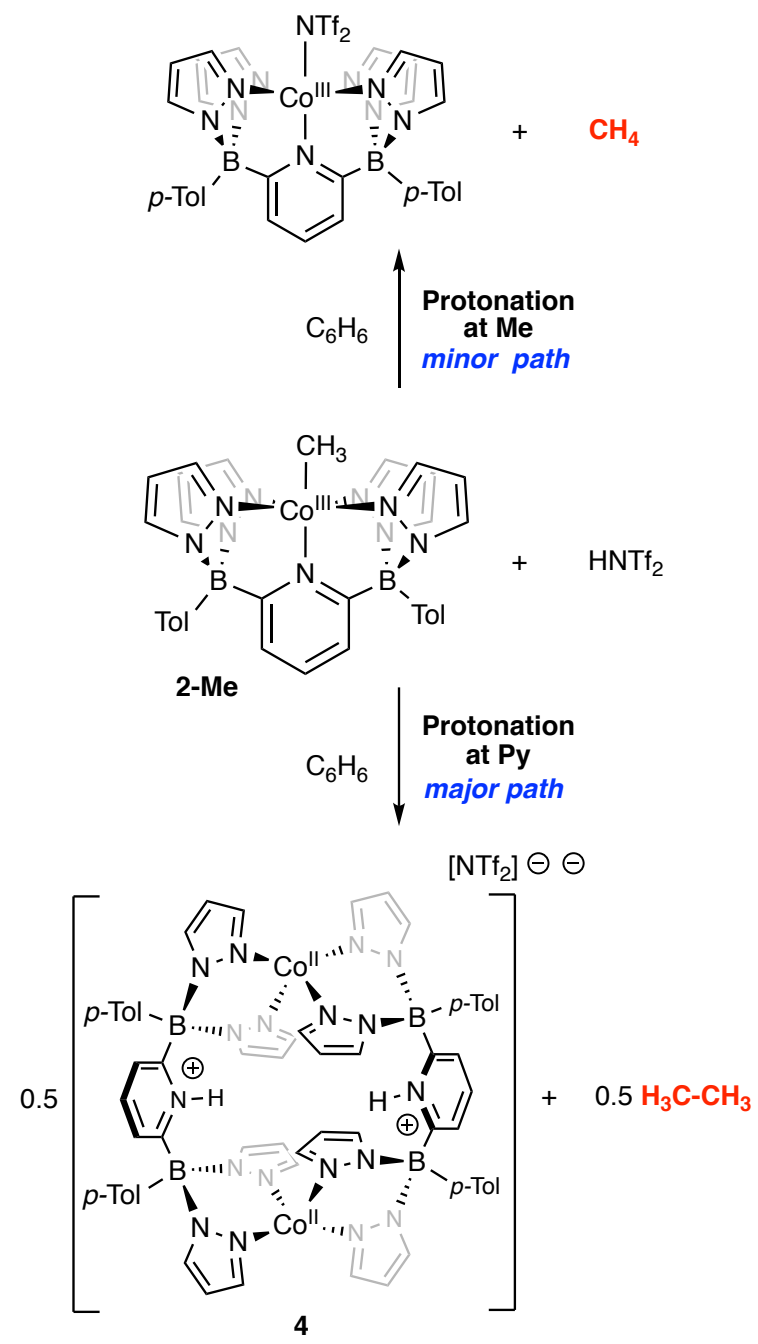

Scheme 7. Divergent protonation pathways for 2-Me. 
The HOMO of 2-Me (Figure S39) is primarily localized along the $\mathrm{Py}-\mathrm{Co}-\mathrm{CH}_{3}$ vector and therefore has electron density associated with both the methyl group and the pyridyl nitrogen. We thus interpret these results in terms of two competing pathways involving protonation of the methyl group leading to $\mathrm{CH}_{4}$ and the contact ion pair $\left[\left(\mathrm{B}_{2} \mathrm{Pz}_{4} \mathrm{Py}\right) \mathrm{Co}(\mathrm{III})\right]^{+}\left[\mathrm{NTf}_{2}\right]^{-}$(minor) and protonation of the pyridyl nitrogen, leading dimeric compound 4 and $\mathrm{H}_{3} \mathrm{C}-\mathrm{CH}_{3}$ (major). Ethane has been observed to form upon photolysis of methyl cobaloximes and labeling experiments indicate that it forms from dimerization of methyl radicals. ${ }^{42}$ While it is not clear exactly how $\mathbf{4}$ forms, we note that protonation of the five coordinate $\mathrm{Co}(\mathrm{II})$ complex $\mathbf{3}$ with $\mathrm{HNTf}_{2}$ gives $\mathbf{4}$ in good yield, suggesting that protonation of the pyridyl nitrogen in 2-Me triggers the homolytic cleavage of the Co-C bond to release $\bullet \mathrm{CH}_{3}$, which dimerizes to ethane. The protonated $\mathrm{Co}(\mathrm{II})$ compound that

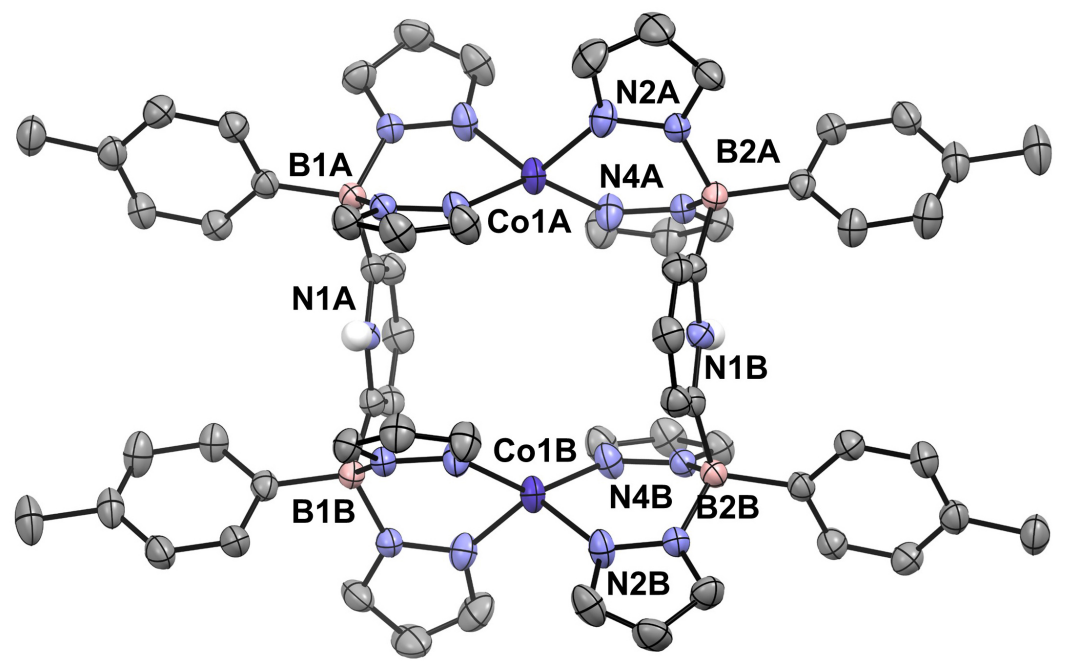

Figure 4. Thermal ellipsoid diagram for 4. Thermal ellipsoids are shown at the $50 \%$ probability level. Calculated hydrogen atoms are omitted for clarity except the ones of the protonated pyridines. The two $\mathrm{NTf}_{2}$ anions are omitted for clarity. Selected bond distances $(\AA ̊)$ and bond angles ( ${ }^{\circ}$ ): Co1-N2, 1.980(6); Co1-N4, 1.981(7); Co1-Co1', 5.738(2); Co1'-N2', 1.977(7); Co1'-N4', 1.982(7). Further metrical data are given in Table S2.

results then dimerizes with ligand rearrangement to give the observed product 4 . The structure of $\mathbf{4}$ is reminiscent of the coordination mode found in in the lithium salts of the ligands that we have observed previously; ${ }^{22}$ a thermal ellipsoid representation of its structure is given in Figure 4, along with some selected metrical parameters, which are 
unremarkable. Interestingly, when $\mathbf{4}$ is treated with LiOiPr in THF, rapid reformation of 3THF is observed. Evidently the substitutional lability of Co(II) compounds enables facile conversion between dinuclear and mononuclear compounds in this system.

\section{Conclusions.}

Cobalt (III) alkyl derivatives have a place of significance in the history of bioorganometallic chemistry due to their role as models for vitamin B12 chemistry. We therefore were interested in preparing alkyl complexes using our recently reported dianionic, electron rich pentadentate diborate platform ${ }^{20}$ to examine their properties in this context but also as potential synthons for incorporating $\left(\mathrm{B}_{2} \mathrm{Pz}_{4} \mathrm{Py}\right) \mathrm{Co}$ units into dinuclear structures. The compounds $2-\mathbf{R}$ are straightforwardly synthesized in good to excellent yields via salt metathesis between Co(III) bromide $\mathbf{1}$ and alkyllithium reagents. The dianionic nature of the ligand stabilizes the Co(III) oxidation state and the alkyl compounds are remarkably robust, thermally and with respect to ambient atmosphere. Nonetheless, there is evidence for reversible bond homolysis in the reaction of compounds 2-R with hydrogen atom donors like 1,4-cyclohexadiene or radical traps like TEMPO, and also in the observed slow cyclization of the "radical clock" 5-hexenyl group to the methylcyclopentyl derivative. Most interestingly, reduction of the Co(III) alkyls to Co(II) anions does not accelerate bond homolysis as previously observed, ${ }^{34}$ but leads to ejection of $\mathrm{R}^{-}$through heterolytic cleavage of the Co-C bond. This is a rare, possibly unprecedented, reactivity mode for Co(III) alkyls and again reflects the unusually electron rich nature of the $\mathrm{B}_{2} \mathrm{Pz}_{4} \mathrm{Py}$ ligand framework, which is not prone to supporting Co(I) centers. This study underscores the utility of this ligand platform for stabilizing higher oxidation state complexes and intermediates in metals from across the transition series.

\section{Experimental Section.}

General Procedures. Unless mentioned otherwise, all manipulations were performed under an inert gas (argon) in gloveboxes or using standard Schlenk techniques. All anhydrous solvents were prepared by passing through Mbraun SP-800 solvent purification 
system and were stored in $500 \mathrm{~mL}$ thick-walled vessels over sodium/benzophenone ketal or $\mathrm{CaH}_{2}$. All dried solvents were degassed, and vacuum distilled prior to use. Pentane, toluene and benzene solvents were dried over $\mathrm{K} / \mathrm{Na}$ alloy and freshly distilled before use. Chemicals were obtained from common vendors and used as received. The syntheses of the dianionic pentadentate $\mathrm{B}_{2} \mathrm{Pz}_{4} \mathrm{Py}$ ligand, $\mathbf{1}, 3$ and $\left[\left(\mathrm{B}_{2} \mathrm{Pz}_{4} \mathrm{Py}\right) \mathrm{Co}(\mathrm{III})\right]^{+}\left[\mathrm{NTf}_{2}\right]^{-}$have previously been reported. ${ }^{22} \mathrm{LiCH}_{2} \mathrm{SiMe}_{3},{ }^{43}$ and $\mathrm{LiCH}_{2} \mathrm{SiMe}_{2} \mathrm{Ph}$, ${ }^{44}$ were prepared according to literature procedures. $\mathrm{Li}^{i} \mathrm{Bu}$ was synthesized using the same procedure as for $\mathrm{LiCH}_{2} \mathrm{SiMe}_{3}$.

Synthesis of 2-Me. To a pressure tube equipped with a Teflon tap containing suspension of 1 (60 mg, $0.087 \mathrm{mmol}$ ) in $8 \mathrm{~mL}$ of toluene, $\mathrm{LiCH}_{3}(2 \mathrm{mg}, 0.087 \mathrm{mmol}$ ) was added as a solid all at once. The resulting orange reaction mixture was stirred for $4 \mathrm{~h}$ at room temperature. The solution was stored at $-40^{\circ} \mathrm{C}$ overnight and passed through a syringe filter. The solvent was removed in vacuo. The orange solid was washed with $5 \mathrm{~mL}$ of pentane and dried in vacuo to afford $38 \mathrm{mg}$ of 2-Me (70\%). Crystals suitable for X-ray diffraction were grown by slow diffusion of pentane in saturated solution of 2-Me in benzene. ${ }^{1} \mathrm{H}$ NMR $(500 \mathrm{MHz}$, $\left.\mathrm{C}_{6} \mathrm{D}_{6}\right) \delta 7.96\left(4 \mathrm{H}, \mathrm{d},{ }^{3} \mathrm{~J}_{\mathrm{HH}}=7.6 \mathrm{~Hz}, \mathrm{H} 5\right), 7.90\left(2 \mathrm{H}, \mathrm{d},{ }^{3} J_{\mathrm{HH}}=7.5 \mathrm{~Hz}, \mathrm{H} 2\right), 7.67(4 \mathrm{H}, \mathrm{m}, \mathrm{H} 9), 7.44$ (4H, m, H11), $7.26\left(4 \mathrm{H}, \mathrm{d},{ }^{3} \mathrm{~J}_{\mathrm{HH}}=7.5 \mathrm{~Hz}, \mathrm{H} 6\right), 7.11\left(1 \mathrm{H}, \mathrm{t},{ }^{3} J_{\mathrm{HH}}=7.5 \mathrm{~Hz}, \mathrm{H} 3\right), 5.78(4 \mathrm{H}, \mathrm{m}$, H10), 4.13 (3H, s, H12), 2.35 (6H, s, H8). ${ }^{13} \mathrm{C}\left\{{ }^{1} \mathrm{H}\right\}$ NMR (151 MHz, $\left.\mathrm{C}_{6} \mathrm{D}_{6}\right) \delta 172.4$ (s, C1), 142.2 (s, C9), 139.9 (s, C4), 137.1 (s, C11), 136.8 (s, C7), 136.0 (s, C5), 133.8 (s, C3), 129.1 (s, C6), 126.8 (s, C2), 105.8 (s, C10), 21.4 (s, C8), 19.1 (s, C12). ${ }^{11} \mathrm{~B}$ NMR (161 MHz, C6 $\mathrm{D}_{6}$ ) $\delta$ 2.1 (s, 2B). Anal. Calcd for $\mathrm{C}_{32} \mathrm{H}_{32} \mathrm{~B}_{2} \mathrm{CoN}_{9}$ : C 61.67; H 5.18; N 20.23. Found: (\%): C 60.25; $\mathrm{H}$ 4.78; $\mathrm{N}$ 19.82. HRMS (APCI) m/z calcd: $624.2372(\mathrm{M}+\mathrm{H})^{+} \mathrm{m} / \mathrm{z}$ found: $624.2400(\mathrm{M}+\mathrm{H})^{+}$.

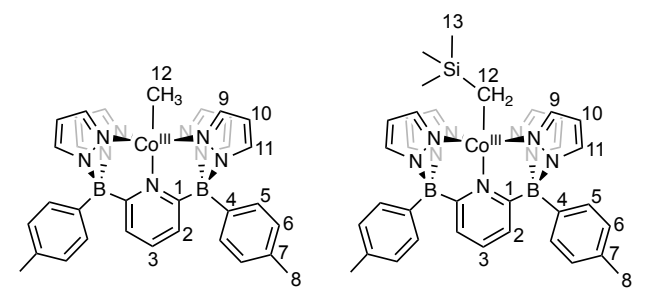

Synthesis of 2- $\mathrm{CH}_{2} \mathrm{SiMe}_{3}$. To a pressure tube equipped with a Teflon tap containing suspension of 1 ( $60 \mathrm{mg}, 0.087 \mathrm{mmol}$ ) in $8 \mathrm{~mL}$ of toluene, $\mathrm{LiCH}_{2} \mathrm{SiMe}_{3}(9 \mathrm{mg}, 0.087 \mathrm{mmol}$ ) dissolved in $2 \mathrm{~mL}$ of toluene was added dropwise. The resulting pink reaction mixture was stirred for $1 \mathrm{~h}$ at room temperature. The solution was stored at $-40^{\circ} \mathrm{C}$ overnight and passed through a syringe filter. The solvent was removed in vacuo. The pink solid was washed with 
$5 \mathrm{~mL}$ of pentane and dried in vacuo to afford $53 \mathrm{mg}$ of $\mathbf{2}-\mathbf{C H}_{2} \mathbf{S i M e}_{3}$ (88\%). Crystals suitable for X-ray diffraction were grown by slow diffusion of pentane in saturated solution of 2$\mathrm{CH}_{2} \mathrm{SiMe}_{3}$ in benzene. ${ }^{1} \mathrm{H}$ NMR $\left(500 \mathrm{MHz}, \mathrm{C}_{6} \mathrm{D}_{6}\right) \delta 7.97\left(4 \mathrm{H}, \mathrm{d},{ }^{3} \mathrm{~J}_{\mathrm{HH}}=7.6 \mathrm{~Hz}, \mathrm{H} 5\right), 7.94(2 \mathrm{H}, \mathrm{d}$, $\left.{ }^{3} \mathrm{~J}_{\mathrm{HH}}=7.6 \mathrm{~Hz}, \mathrm{H} 2\right), 7.86\left(4 \mathrm{H}, \mathrm{d},{ }^{3} \mathrm{~J}_{\mathrm{HH}}=2.4 \mathrm{~Hz}, \mathrm{H} 9\right), 7.40\left(4 \mathrm{H}, \mathrm{d},{ }^{3} \mathrm{~J}_{\mathrm{HH}}=2.4 \mathrm{~Hz}, \mathrm{H} 11\right), 7.25(4 \mathrm{H}, \mathrm{d}$, $\left.{ }^{3} \mathrm{~J}_{\mathrm{HH}}=7.6 \mathrm{~Hz}, \mathrm{H6}\right), 7.10\left(1 \mathrm{H}, \mathrm{t},{ }^{3} \mathrm{~J}_{\mathrm{HH}}=7.6 \mathrm{~Hz}, \mathrm{H} 3\right), 5.77\left(4 \mathrm{H}, \mathrm{t},{ }^{3} \mathrm{~J}_{\mathrm{HH}}=2.4 \mathrm{~Hz}, \mathrm{H} 10\right), 4.10(2 \mathrm{H}, \mathrm{s}$, H12), $2.34(6 \mathrm{H}, \mathrm{s}, \mathrm{H} 8),-0.09(9 \mathrm{H}, \mathrm{s}, \mathrm{H} 13) .{ }^{13} \mathrm{C}\left\{{ }^{1} \mathrm{H}\right\} \mathrm{NMR}\left(151 \mathrm{MHz}, \mathrm{C}_{6} \mathrm{D}_{6}\right) \delta 172.5$ (s, C1), 143.0 (s, C9), 139.7 (s,C4), 137.5 (s, C11), 136.9 (s, C5), 136.1 (s, C7), 133.7 (s, C3), 129.2 (s, C6), 126.5 (s, C2), 105.8 (s, C10), 21.4 (s, C8), 16.6 (s, C12), 1.53 (s, C13). ${ }^{11}$ B NMR (161 $\left.\mathrm{MHz}, \mathrm{C}_{6} \mathrm{D}_{6}\right) \delta-1.6$ (s, 2B). Anal. Calcd for $\mathrm{C}_{35} \mathrm{H}_{40} \mathrm{~B}_{2} \mathrm{SiCoN}_{9}$ : C 60.45; H 5.80; N 18.13. Found: (\%): C 60.82; H 5.62; N 17.96.

Synthesis of 2-CH $\mathbf{C H}_{2} \mathrm{SiMe}_{2} \mathrm{Ph}$. To a pressure tube equipped with a Teflon tap containing suspension of 1 ( $60 \mathrm{mg}, 0.087 \mathrm{mmol}$ ) in $8 \mathrm{~mL}$ of toluene, $\mathrm{LiCH}_{2} \mathrm{SiMe}_{2} \mathrm{Ph}(14 \mathrm{mg}, 0.087$ mmol) dissolved in $2 \mathrm{~mL}$ of toluene was added dropwise. The resulting light pink reaction mixture was stirred for $1 \mathrm{~h}$ at room temperature. The solution was stored at $-40^{\circ} \mathrm{C}$ overnight and passed through a syringe filter. The solvent was removed in vacuo. The pink solid was washed with $5 \mathrm{~mL}$ of pentane and dried in vacuo to afford $47 \mathrm{mg}$ of 2$\mathbf{C H}_{2} \mathbf{S i M e}_{2} \mathbf{P h}(71 \%)$. Crystals suitable for X-ray diffraction were grown by slow diffusion of pentane in saturated solution of $\mathbf{2}-\mathbf{C H}_{2} \mathbf{S i M e} 2 \mathbf{P h}$ in benzene. ${ }^{1} \mathrm{H}$ NMR $\left(500 \mathrm{MHz}, \mathrm{C}_{6} \mathrm{D}_{6}\right) \delta 7.97$ $\left(4 \mathrm{H}, \mathrm{d},{ }^{3} J_{\mathrm{HH}}=7.6 \mathrm{~Hz}, \mathrm{H} 5\right), 7.92\left(2 \mathrm{H}, \mathrm{d},{ }^{3} J_{\mathrm{HH}}=7.6 \mathrm{~Hz}, \mathrm{H} 2\right), 7.83(2 \mathrm{H}, \mathrm{m}, \mathrm{H} 15), 7.74\left(4 \mathrm{H}, \mathrm{d},{ }^{3} J_{\mathrm{HH}}\right.$ $=2.4 \mathrm{~Hz}, \mathrm{H} 9), 7.39\left(4 \mathrm{H}, \mathrm{d},{ }^{3} J_{\mathrm{HH}}=2.4 \mathrm{~Hz}, \mathrm{H} 11\right), 7.37\left(2 \mathrm{H}, \mathrm{d},{ }^{3} J_{\mathrm{HH}}=7.4 \mathrm{~Hz}, \mathrm{H} 16\right), 7.32(1 \mathrm{H}, \mathrm{t}$, $\left.{ }^{3} J_{\mathrm{HH}}=7.4 \mathrm{~Hz}, \mathrm{H} 17\right), 7.26\left(4 \mathrm{H}, \mathrm{d},{ }^{3} J_{\mathrm{HH}}=7.6 \mathrm{~Hz}, \mathrm{H} 6\right), 7.08\left(1 \mathrm{H}, \mathrm{t},{ }^{3} \mathrm{JHH}_{\mathrm{HH}}=7.6 \mathrm{~Hz}, \mathrm{H} 3\right), 5.72(4 \mathrm{H}, \mathrm{t}$, $\left.{ }^{3} J_{\mathrm{HH}}=2.4 \mathrm{~Hz}, \mathrm{H} 10\right), 4.27(2 \mathrm{H}, \mathrm{s}, \mathrm{H} 12), 2.34(6 \mathrm{H}, \mathrm{s}, \mathrm{H} 8),-0.17(6 \mathrm{H}, \mathrm{s}, \mathrm{H} 13) .{ }^{13} \mathrm{C}\left\{{ }^{1} \mathrm{H}\right\}$ NMR $(151$ MHz, $\mathrm{C}_{6} \mathrm{D}_{6}$ ) $\delta 144.6$ (s, C14), 143.3 (s, C9), 137.5 (s, C11), 136.9 (s, C7), 136.0 (s, C5), 134.5 (s, C15), 133.7 (s, C3), 129.2 (s, C6), 128.7 (s, C16), 128.4 (s, C17), 126.5 (s, C2), 105.8 (s, C10), 21.4 (s, C8), 14.3 (s, C12), -1.2 (s, C13). C1 and C4 not observed. ${ }^{11}$ B NMR (161 MHz, $\left.\mathrm{C}_{6} \mathrm{D}_{6}\right) \delta-1.5$ (s, 2B). Anal. Calcd for $\mathrm{C}_{40} \mathrm{H}_{42} \mathrm{~B}_{2} \mathrm{CoN}_{9} \mathrm{Si}$ : C 63.43; H 5.59; N 16.64. Found: (\%): C 62.58; H 5.25; N 16.40. 


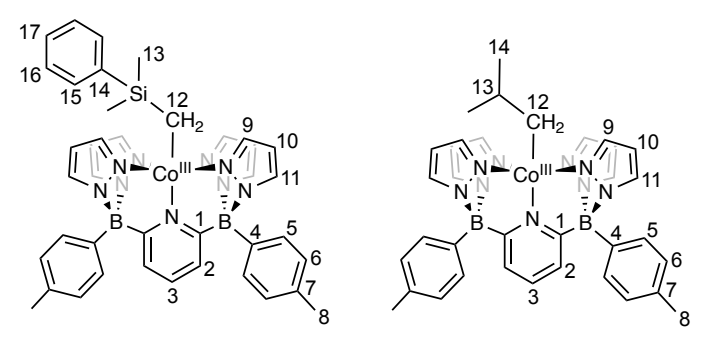

Synthesis of 2-iBu. To a pressure tube equipped with a Teflon tap containing suspension of 1 ( $60 \mathrm{mg}, 0.087 \mathrm{mmol}$ ) in $8 \mathrm{~mL}$ of toluene, LiiBu ( $9 \mathrm{mg}, 0.087 \mathrm{mmol}$ ) dissolved in $2 \mathrm{~mL}$ of toluene was added dropwise. The resulting pink reaction mixture was stirred for $1 \mathrm{~h}$ at room temperature. The solution was stored at $-40^{\circ} \mathrm{C}$ overnight and passed through a syringe filter. The solvent was removed in vacuo. The pink solid was washed with $5 \mathrm{~mL}$ of pentane and dried in vacuo to afford $35 \mathrm{mg}$ of $\mathbf{2}^{-\mathrm{i}} \mathrm{Bu}(58 \%) .{ }^{1} \mathrm{H}$ NMR $\left(500 \mathrm{MHz}, \mathrm{C}_{6} \mathrm{D}_{6}\right) \delta 7.98$ $\left(4 \mathrm{H}, \mathrm{d},{ }^{3} J_{\mathrm{HH}}=7.6 \mathrm{~Hz}, \mathrm{H} 5\right), 7.92\left(2 \mathrm{H}, \mathrm{d},{ }^{3} J_{\mathrm{HH}}=7.6 \mathrm{~Hz}, \mathrm{H} 2\right), 7.67\left(4 \mathrm{H}, \mathrm{d},{ }^{3} \int_{\mathrm{HH}}=2.4 \mathrm{~Hz}, \mathrm{H} 9\right), 7.44$ $\left(4 \mathrm{H}, \mathrm{d},{ }^{3} J_{\mathrm{HH}}=2.4 \mathrm{~Hz}, \mathrm{H} 11\right), 7.26\left(4 \mathrm{H}, \mathrm{d},{ }^{3} J_{\mathrm{HH}}=7.6 \mathrm{~Hz}, \mathrm{H6}\right), 7.12\left(1 \mathrm{H}, \mathrm{t},{ }^{3} J_{\mathrm{HH}}=7.6 \mathrm{~Hz}, \mathrm{H} 3\right), 5.77$ $\left(4 \mathrm{H}, \mathrm{t}, 3 J_{\mathrm{HH}}=2.4 \mathrm{~Hz}, \mathrm{H} 10\right), 4.88\left(2 \mathrm{H}, \mathrm{d}, 3 J_{\mathrm{HH}}=7.0 \mathrm{~Hz}, \mathrm{H} 12\right), 2.58(1 \mathrm{H}, \mathrm{m}, \mathrm{H} 13), 2.34(6 \mathrm{H}, \mathrm{s}$, H8), $0.98\left(6 \mathrm{H}, \mathrm{d},{ }^{3} \mathrm{JHH}_{\mathrm{HH}}=6.3 \mathrm{~Hz}, \mathrm{H} 14\right) .{ }^{13} \mathrm{C}\left\{{ }^{1} \mathrm{H}\right\} \mathrm{NMR}\left(151 \mathrm{MHz}, \mathrm{C}_{6} \mathrm{D}_{6}\right) \delta 172.5$ (s, C1), 142.5 (s, C9), 140.1 (s, C4), 137.4 (s, C11), 136.8 (s, C7), 136.1 (s, C5), 133.6 (s, C3), 129.1 (s, C6), 126.5 (s, C2), 105.6 (s, C10), 43,7 (s, C12), 32.9 (s, C13), 23,7 (c, C14), 21.4 (s, C8). ${ }^{11}$ B NMR (161 MHz, $\left.\mathrm{C}_{6} \mathrm{D}_{6}\right) \delta-1.6$ (s, 2B). Anal. Calcd for $\mathrm{C}_{35} \mathrm{H}_{38} \mathrm{~B}_{2} \mathrm{CoN}_{9}$ : C 63.19; H 5.76; N 18.95. Found: (\%): C 63.06; H 5.84; $\mathrm{N}$ 18.58. HRMS (APCI) m/z calcd: $666.2841(\mathrm{M}+\mathrm{H})^{+} \mathrm{m} / \mathrm{z}$ found: $666.2823(\mathrm{M}+\mathrm{H})^{+}$.

Synthesis of $2-\mathrm{CH}_{2}\left(c-\mathrm{C}_{5} \mathrm{H}_{9}\right)$. A suspension of $\mathbf{1}(60 \mathrm{mg}, 0.087 \mathrm{mmol})$ in $8 \mathrm{~mL}$ of toluene in a swivel frit apparatus was cooled to $-78^{\circ} \mathrm{C}$ using an acetone/dry ice bath. $272 \mu \mathrm{L}$ of (cyclopentylmethyl)lithium $(0.32 \mathrm{M}, 0.087 \mathrm{mmol})$ in $3: 2$ ratio of pentane:diethyl ether was added dropwise at $-78{ }^{\circ} \mathrm{C}$ under a flow of Ar. The acetone/dry ice bath was removed. The resulting brown reaction mixture was stirred for $1 \mathrm{~h}$ at room temperature. The solution was filtered to remove lithium salts. The solvent was removed in vacuo. The brown solid was washed with $5 \mathrm{~mL}$ of pentane and dried in vacuo to afford $54 \mathrm{mg}$ of $\mathbf{2}-\mathbf{C H}_{2}\left(\boldsymbol{c}-\mathbf{C}_{5} \mathbf{H}_{9}\right)$ (90\%). ${ }^{1} \mathrm{H}$ NMR (500 MHz, C $\left.6 \mathrm{D}_{6}\right) \delta 7.98\left(4 \mathrm{H}, \mathrm{d},{ }^{3} J_{\mathrm{HH}}=7.6 \mathrm{~Hz}, \mathrm{H} 5\right), 7.92\left(2 \mathrm{H}, \mathrm{d},{ }^{3} J_{\mathrm{HH}}=7.6 \mathrm{~Hz}\right.$, H2), $7.70\left(4 \mathrm{H}, \mathrm{d},{ }^{3} J_{\mathrm{HH}}=2.4 \mathrm{~Hz}, \mathrm{H} 9\right), 7.45\left(4 \mathrm{H}, \mathrm{d},{ }^{3} \mathrm{~J}_{\mathrm{HH}}=2.4 \mathrm{~Hz}, \mathrm{H} 11\right), 7.25\left(4 \mathrm{H}, \mathrm{d},{ }^{3} J_{\mathrm{HH}}=7.6 \mathrm{~Hz}\right.$, H6), $7.12\left(1 \mathrm{H}, \mathrm{t},{ }^{3} J_{\mathrm{HH}}=7.6 \mathrm{~Hz}, \mathrm{H} 3\right), 5.78\left(4 \mathrm{H}, \mathrm{t},{ }^{3} J_{\mathrm{HH}}=2.4 \mathrm{~Hz}, \mathrm{H} 10\right), 5.04\left(2 \mathrm{H}, \mathrm{d},{ }^{3} \mathrm{~J}_{\mathrm{HH}}=7.3 \mathrm{~Hz}\right.$, H12), 2.70 (1H, m, H13), 2.34 (6H, s, H8), 1.75 (2H, m, H15), 1.57 (2H, m, H16), 1.51 (2H, m, 
H14), 1.39 (2H, m, H17). ${ }^{13} \mathrm{C}\left\{{ }^{1} \mathrm{H}\right\}$ NMR (151 MHz, C6 $\mathrm{D}_{6}$ ) $\delta 172.6$ (s, C1), 142.5 (s, C9), 139.1 (s, C4), 137.3 (s, C11), 136.8 (s, C7), 136.1 (s, C5), 133.6 (s, C3), 129.1 (s, C6), 126.5 (s, C2), 105.6 (s, C10), 46.2 (s, C13), 40,8 (s, C12), 34.2 (s, C14 and C17), 26,1 (s, C15 and C16), 21.4 (s, C8). ${ }^{11} \mathrm{~B}$ NMR (161 MHz, $\mathrm{C}_{6} \mathrm{D}_{6}$ ) $\delta-1.6$ (s, 2B). Anal. Calcd for $\mathrm{C}_{37} \mathrm{H}_{40} \mathrm{~B}_{2} \mathrm{CoN}_{9}$ : C 64.28; $\mathrm{H}$ 5.83; N 18.23. Found: (\%): C 62.55; H 5.37; N 18.22.
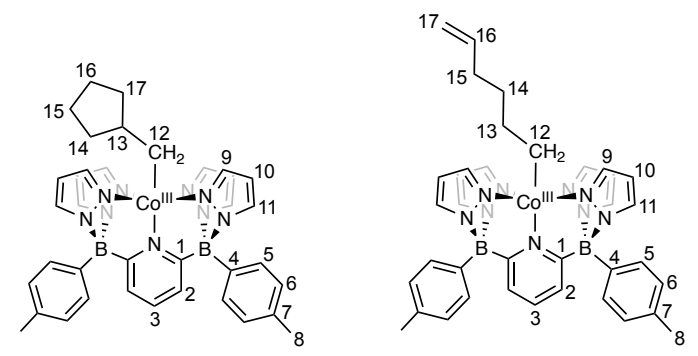

Generation of 2-( $\left(\mathbf{C H}_{2}\right)_{4} \mathbf{C H}=\mathbf{C H}_{2}$. A suspension of $\mathbf{1}(60 \mathrm{mg}, 0.087 \mathrm{mmol})$ in $8 \mathrm{~mL}$ of toluene in a swivel frit apparatus was cooled to $-78 \bullet \mathrm{C}$ using an acetone/dry ice bath. $272 \mu \mathrm{L}$ of 5 Hexenyllithium $(0.32 \mathrm{M}, 0.087 \mathrm{mmol})$ in $3: 2$ ratio of pentane:diethyl ether was added dropwise at $-78^{\circ} \mathrm{C}$ under a flow of Ar. The resulting brown reaction mixture was stirred for $1 \mathrm{~h}$ at $-78^{\circ} \mathrm{C}$. The solution was filtered to remove lithium salts. The solvent was removed in vacuo. The brown solid was washed with $5 \mathrm{~mL}$ of pentane and dried in vacuo to afford 41 $\mathrm{mg}$ of a mixture of the desire product $\mathbf{2}-\left(\mathbf{C H}_{2}\right)_{4} \mathbf{C H}=\mathbf{C H}_{2}$ and $\mathbf{2}-\mathbf{C H}_{2}\left(\boldsymbol{c}-\mathbf{C}_{5} \mathbf{H}_{9}\right)(68 \%)$. The formation of $\mathbf{2}-\mathbf{C H}_{2}\left(\boldsymbol{c}-\mathbf{C}_{5} \mathrm{H}_{9}\right)$ is arising from the generation of cyclo(pentylmethyl)lithium in situ (see Figure S16 for assignment from the mixture). ${ }^{1} \mathrm{H}$ NMR $\left(500 \mathrm{MHz}, \mathrm{C}_{6} \mathrm{D}_{6}\right) \delta 7.98(4 \mathrm{H}$, d, $\left.{ }^{3} J_{\mathrm{HH}}=7.6 \mathrm{~Hz}, \mathrm{H} 5\right), 7.95\left(2 \mathrm{H}, \mathrm{d},{ }^{3} J_{\mathrm{HH}}=7.6 \mathrm{~Hz}, \mathrm{H} 2\right), 7.75\left(4 \mathrm{H}, \mathrm{d},{ }^{3} J_{\mathrm{HH}}=2.4 \mathrm{~Hz}, \mathrm{H9}\right), 7.39(4 \mathrm{H}$, d, $\left.{ }^{3} J_{\mathrm{HH}}=2.4 \mathrm{~Hz}, \mathrm{H} 11\right), 7.25\left(4 \mathrm{H}, \mathrm{d},{ }^{3} J_{\mathrm{HH}}=7.6 \mathrm{~Hz}, \mathrm{H6}\right), 7.12\left(1 \mathrm{H}, \mathrm{t},{ }^{3} J_{\mathrm{HH}}=7.6 \mathrm{~Hz}, \mathrm{H} 3\right), 5.95(2 \mathrm{H}$, m, H12), $5.79\left(4 \mathrm{H}, \mathrm{t}, 3_{\mathrm{HH}}=2.4 \mathrm{~Hz}, \mathrm{H} 10\right), 5.10(2 \mathrm{H}, \mathrm{m}, \mathrm{H} 17), 2.67(1 \mathrm{H}, \mathrm{m}, \mathrm{H} 16), 2.34(6 \mathrm{H}, \mathrm{s}$, H8), $2.25(2 \mathrm{H}, \mathrm{m}, \mathrm{H} 15), 1.93(2 \mathrm{H}, \mathrm{m}, \mathrm{H} 14), 1.72(2 \mathrm{H}, \mathrm{m}, \mathrm{H} 13) .{ }^{13} \mathrm{C}\left\{{ }^{1} \mathrm{H}\right\} \mathrm{NMR}\left(151 \mathrm{MHz}, \mathrm{C}_{6} \mathrm{D}_{6}\right)$ $\delta 142.6$ (s, C9), 139.9 (s, C4), 137.3 (s, C11), 136.8 (s, C7), 136.2 (s, C5), 133.6 (s, C3), 129.1 (s, C6), 126.4 (s, C2), 105.7 (s, C10), 46.2 (s, C12), 34.5 (s, C17), 34.2 (s, C15), 31.4 (s, C14), 26.1, (s, C13), 21.4 (s, C8). C1 not observed. ${ }^{11} \mathrm{~B}$ NMR (161 MHz, $\left.\mathrm{C}_{6} \mathrm{D}_{6}\right) \delta-1.7$ (s, 2B). Anal. Calcd for $\mathrm{C}_{37} \mathrm{H}_{40} \mathrm{~B}_{2} \mathrm{CoN}_{9}$ : C 64.28; H 5.83; N 18.23. Found: (\%): C 62.57; H 5.44; N 17.49.

Generation of 4. To a pressure tube equipped with a Teflon tap containing suspension of $\mathbf{3}$ ( $60 \mathrm{mg}, 0.099 \mathrm{mmol}$ ) in $10 \mathrm{~mL}$ of bromobenzene, $\mathrm{HNTf}_{2}$ (28 $\mathrm{mg}, 0.099 \mathrm{mmol}$ ) was added as a solid all at once at room temperature. The reaction was immediate, resulting in the 
formation of a deep purple precipitate. The solvent was removed in vacuo to afford $75 \mathrm{mg}$ of $4(85 \%)$. Crystals suitable for X-ray diffraction were obtained from the precipitate formed in bromobenzene. ${ }^{1} \mathrm{H}$ NMR $\left(500 \mathrm{MHz}, o-\mathrm{Cl}_{2}-\mathrm{C}_{6} \mathrm{D}_{4}\right) \delta 47.5,35.0,31.8,27.2,24.5,22.3$, 16.0, 13.8, 10.2, 6.0, 1.3, 0.9, -35.7, -39.6. ${ }^{19} \mathrm{~F}$ NMR (471 MHz, $\left.o-\mathrm{Cl}_{2}-\mathrm{C}_{6} \mathrm{D}_{4}\right) \delta-79.4$ (s, 12F) No ${ }^{11} \mathrm{~B}$ signal. Anal. Calcd for $\mathrm{C}_{66} \mathrm{H}_{60} \mathrm{~B}_{4} \mathrm{Co}_{2} \mathrm{~N}_{20} \mathrm{~F}_{12} \mathrm{O}_{8} \mathrm{~S}_{4}$ : C 44.57; H 3.40; N 15.75. Found: (\%): C 45.05; $\mathrm{H} 3.68 ; \mathrm{N} 15.17$. Note: 4 is sparingly soluble in $o-\mathrm{Cl}_{2}-\mathrm{C}_{6} \mathrm{D}_{4}$.

\section{Supporting Information Available.}

The following files are available free of charge.

Experimental and characterization details for all new compounds, including spectroscopic data, X-ray crystallographic data and computational details with the cartesian coordinates for calculated structures (PDF).

CCDC codes 1993721-1993724 contain the crystallographic data for compounds 2-Me, 2$\mathrm{CH}_{2} \mathrm{SiMe}_{3}, 2-\mathrm{CH}_{2} \mathrm{SiMe}_{2} \mathrm{Ph}$ and 4. These data can be obtained free of charge via www.ccdc.cam.ac.uk/data_request/cif, or by emailing data_request@ccdc.cam.ac.uk, or by contacting The Cambridge Crystallographic Data Centre, 12 Union Road, Cambridge CB2 1EZ, UK; fax: +44 1223336033

\section{Acknowledgements.}

W.E.P thanks the Canada Research Chair secretariat for a Tier I CRC (2013-2027). Funding for the experimental work described was provided by the Natural Sciences and Engineering Research Council of Canada in the form of a Discovery Grant to W.E.P. L.N. thanks Alberta Innovates Technology Futures and the Vanier Canada Graduate Scholarships for support. 


\section{References.}

1. Lindstrand, K. ISOLATION OF METHYLCOBALAMIN FROM NATURAL SOURCE MATERIAL. Nature 1964, 204, 188-189.

2. Barker, H. A.; Weissbach, H.; Smyth, R. D. A COENZYME CONTAINING PSEUDOVITAMIN B12. Proc. Natl. Acad. Sci. 1958, 44, 1093-1097.

3. Giedyk, M.; Goliszewska, K.; Gryko, D. Vitamin B-12 catalysed reactions. Chem. Soc. Rev. 2015, 44, 3391-3404.

4. Brown, K. L. Chemistry and enzymology of vitamin B-12. Chem. Rev. 2005, 105, 20752149.

5. Chandra, T.; Brown, K. L. Vitamin B-12 and alpha-ribonudeosides. Tetrahedron 2008, 64, 9-38.

6. Demarteau, J.; Debuigne, A.; Detrembleur, C. Organocobalt Complexes as Sources of Carbon-Centered Radicals for Organic and Polymer Chemistries. Chem. Rev. 2019, 119, 6906-6955.

7. Grohmann, A. Tetrapodal pentadentate ligands: Single site reactivity and bond activation in iron(II) complexes. Dalton Trans. 2010, 39, 1432-1440.

8. Blackman, A. G. The coordination chemistry of acyclic pentadentate pentaamine ligands. Polyhedron 2019, 161, 1-33.

9. Concepcion, J. J.; Jurss, J. W.; Templeton, J. L.; Meyer, T. J. One Site is Enough. Catalytic Water Oxidation by $\mathrm{Ru}(\mathrm{tpy})(\mathrm{bpm})(\mathrm{OH} 2)(2+)$ and $\mathrm{Ru}(\mathrm{tpy})(\mathrm{bpz})(\mathrm{OH} 2)(2+)$. J. Am. Chem. Soc. 2008, 130, 16462-16463.

10. Sun, Y.; Bigi, J. P.; Piro, N. A.; Tang, M. L.; Long, J. R.; Chang, C. J. Molecular Cobalt Pentapyridine Catalysts for Generating Hydrogen from Water. J. Am. Chem. Soc. 2011, 133, 9212-9215.

11. Zee, D. Z.; Chantarojsiri, T.; Long, J. R.; Chang, C. J. Metal-Polypyridyl Catalysts for Electro- and Photochemical Reduction of Water to Hydrogen. Acc. Chem. Res. 2015, 48, 2027-2036.

12. Nippe, M.; Khnayzer, R. S.; Panetier, J. A.; Zee, D. Z.; Olaiya, B. S.; Head-Gordon, M.; Chang, C. J.; Castellano, F. N.; Long, J. R. Catalytic proton reduction with transition metal complexes of the redox-active ligand bpy2PYMe. Chem. Sci. 2013, 4, 3934-3945. 
13. Song, X. W.; Meng, Y.; Zhang, C. L.; Ma, C. B.; Chen, C. N. A cobalt complex of a pentadentate aminopyridine ligand as an efficient catalyst for photocatalytic hydrogen generation. Inorg. Chem. Comm. 2017, 76, 52-54.

14. Li, J.; Guttinger, R.; More, R.; Song, F.; Wan, W.; Patzke, G. R. Frontiers of water oxidation: the quest for true catalysts. Chem. Soc. Rev. 2017, 46, 6124-6147.

15. Wasylenko, D. J.; Ganesamoorthy, C.; Koivisto, B. D.; Henderson, M. A.; Berlinguette, C. P. Insight into Water Oxidation by Mononuclear Polypyridyl Ru Catalysts. Inorg. Chem. 2010, 49, 2202-2209.

16. Grohmann, A.; Heinemann, F. W.; Kofod, P. The methylcobalt(III) complex of a tetrapodal pentadentate amine ligand, 2,6-bis $\left(1^{\prime}\right.$, $3^{\prime}$-diamino- $2^{\prime}$-methyl-prop-2' yl)pyridine. Inorg. Chim. Acta 1999, 286, 98-102.

17. E. de Vries, M.; M. La Crois, R.; Roelfes, G.; Kooijman, H.; L. Spek, A.; Hage, R.; L. Feringa, B. A novel pentadentate ligand 2,6-bis[methoxybis(2-pyridyl)methyl]pyridine L for mononuclear iron(II) and manganese(II) compounds; synthesis and crystal structures of [FeL(MeCN)][ClO4]2 and [(MnL(H2O)][ClO4]2. Chem. Comm. 1997, 1549-1550.

18. Jonas, R. T.; Stack, T. D. P. C-H Bond Activation by a Ferric Methoxide Complex: A Model for the Rate-Determining Step in the Mechanism of Lipoxygenase. J. Am. Chem. Soc. 1997, 119, 8566-8567.

19. Morin, T. J.; Bennett, B.; Lindeman, S. V.; Gardinier, J. R. First-Row Transition-Metal Complexes of a New Pentadentate Ligand, $\alpha, \alpha, \alpha^{\prime}, \alpha^{\prime}$-Tetra(pyrazolyl)lutidine. Inorg. Chem. 2008, 47, 7468-7470.

20. Spasyuk, D. M.; Carpenter, S. H.; Kefalidis, C. E.; Piers, W. E.; Neidig, M. L.; Maron, L. Facile hydrogen atom transfer to iron(III) imido radical complexes supported by a dianionic pentadentate ligand. Chem. Sci. 2016, 7, 5939-5944.

21. Nurdin, L.; Spasyuk, D. M.; Piers, W. E.; Maron, L. Reactions of Neutral Cobalt(II) Complexes of a Dianionic Tetrapodal Pentadentate Ligand: Cobalt(III) Amides from Imido Radicals. Inorg. Chem. 2017, 56, 4157-4168.

22. Nurdin, L.; Spasyuk, D. M.; Fairburn, L.; Piers, W. E.; Maron, L. Oxygen-Oxygen Bond Cleavage and Formation in Co(II)-Mediated Stoichiometric 0-2 Reduction via the 
Potential Intermediacy of a Co(IV) Oxyl Radical. J. Am. Chem. Soc. 2018, 140, 1609416105.

23. Kendrick, M. J.; Alakhdar, W. PREPARATION AND CHARACTERIZATION OF (ALKYLPEROXO)COBALT(III) PORPHYRINS - 1ST DIRECT EVIDENCE FOR METALCARBON BOND HEMOLYSIS IN DIOXYGEN INSERTION REACTION. Inorg. Chem. 1987, 26, 3971-3972.

24. Tokuyasu, T.; Kunikawa, S.; Masuyama, A.; Nojima, M. Co(III)-alkyl complex- and Co(III)alkylperoxo complex-catalyzed triethylsilylperoxidation of alkenes with molecular oxygen and triethylsilane. Org. Lett. 2002, 4, 3595-3598.

25. Kofod, P.; Harris, P.; Larsen, S. NMR spectroscopic characterization of methylcobalt(III) compounds with classical ligands. Crystal structures of $\mathrm{Co}(\mathrm{NH} 3)(5)(\mathrm{CH} 3) \mathrm{S} 2 \mathrm{O6}$, transCo(en)(2)(NH3)(CH3) S2O6 (en equals 1,2-ethanediamine), and Co(NH3)(6) mer,trans- $\mathrm{Co}(\mathrm{NO} 2)(3)(\mathrm{NH} 3)(2)(\mathrm{CH} 3)(2)$-trans- $\mathrm{Co}(\mathrm{NO} 2)(4)(\mathrm{NH} 3)(2)$. Inorg. Chem. 1997, 36, 2258-2266.

26. Reich, H. J. 6-CMR-5 One-Bond Carbon-Proton Coupling (1JCH). https://www.chem.wisc.edu/areas/reich/nmr/06-cmr-05-1jch.htm (February 1, 2020).

27. Dreos, R.; Felluga, A.; Nardin, G.; Randaccio, L.; Sandri, M.; Tauzher, G. New alkylcobalt(III) complexes containing chiral centers in the chelating system. Inorg. Chem. 2002, 41, 4548-4554.

28. Galezowski, W.; Kubicki, M. X-ray structures and homolysis of some alkylcobalt(III) phthalocyanine complexes. Inorg. Chem. 2005, 44, 9902-9913.

29. Rossi, M.; Glusker, J. P.; Randaccio, L.; Summers, M. F.; Toscano, P. J.; Marzilli, L. G. THE STRUCTURE OF A B-12 COENZYME - METHYLCOBALAMIN STUDIES BY X-RAY AND NMR METHODS. J. Am. Chem. Soc. 1985, 107, 1729-1738.

30. Quagliano, J. V.; Schubert, L. E. O. The Trans Effect in Complex Inorganic Compounds. Chem. Rev. 1952, 50, 201-260.

31. Beh, D. W.; Piers, W. E.; del Rosal, I.; Maron, L.; Gelfand, B. S.; Gendy, C.; Lin, J. B. Scandium alkyl and hydride complexes supported by a pentadentate diborate ligand: reactions with CO2 and N2O. Dalton Trans. 2018, 47, 13680-13688. 
32. Beh, D. W.; Piers, W. E.; Maron, L.; Yang, Y.; Gelfand, B. S.; Li, J.-B. Hydrolysis of scandium alkyl derivatives supported by a pentadentate diborate ligand: Interconversion of hydroxo and oxo complexes. Polyhedron 2020, 179, 114410.

33. Tsou, T. T.; Loots, M.; Halpern, J. KINETIC DETERMINATION OF TRANSITION-METAL ALKYL BOND-DISSOCIATION ENERGIES - APPLICATION TO ORGANOCOBALT COMPOUNDS RELATED TO B-12 COENZYMES. J. Am. Chem. Soc. 1982, 104, 623-624.

34. Martin, B. D.; Finke, R. G. METHYLCOBALAMINS FULL-STRENGTH VS HALF-STRENGTH COBALT-CARBON SIGMA-BONDS AND BOND-DISSOCIATION ENTHALPIES - AGREATER-THAN-10(15) CO-CH3 HOMOLYSIS RATE ENHANCEMENT FOLLOWING ONE-ANTIBONDING-ELECTRON REDUCTION OF METHYLCOBALAMIN. J. Am. Chem. Soc. 1992, 114, 585-592.

35. Hay, B. P.; Finke, R. G. THERMOLYSIS OF THE CO-C BOND IN ADENOSYLCORRINS .3. QUANTIFICATION OF THE AXIAL BASE EFFECT IN ADENOSYLCOBALAMIN BY THE SYNTHESIS AND THERMOLYSIS OF AXIAL BASE-FREE ADENOSYLCOBINAMIDE INSIGHTS INTO THE ENERGETICS OF ENZYME-ASSISTED COBALT CARBON BOND HOMOLYSIS. J. Am. Chem. Soc. 1987, 109, 8012-8018.

36. Koide, T.; Aritome, I.; Saeki, T.; Morita, Y.; Shiota, Y.; Yoshizawa, K.; Shimakoshi, H.; Hisaeda, Y. Cobalt-Carbon Bond Formation Reaction via Ligand Reduction of Porphycene-Cobalt(II) Complex and Its Noninnocent Reactivity. ACS Omega 2018, 3, 4027-4034.

37. Cowley, R. E.; Eckert, N. A.; Vaddadi, S.; Figg, T. M.; Cundari, T. R.; Holland, P. L. Selectivity and Mechanism of Hydrogen Atom Transfer by an Isolable Imidoiron(III) Complex. J. Am. Chem. Soc. 2011, 133, 9796-9811.

38. Griller, D.; Ingold, K. U. FREE-RADICAL CLOCKS. Acc. Chem. Res. 1980, 13, 317-323.

39. Bailey, W. F.; Patricia, J. J.; Delgobbo, V. C.; Jarret, R. M.; Okarma, P. J. CYCLIZATION OF 5HEXENYLLITHIUM TO (CYCLOPENTYLMETHYL)LITHIUM. J. Org. Chem. 1985, 50, 19992000.

40. Lehmkuhl, H.; Nehl, H. F. Über (Cyclopentadienyl)organylcobalt-Komplexe. Chem. Ber. 1984, 117, 3443-3456.

41. Fulmer, G. R.; Miller, A. J. M.; Sherden, N. H.; Gottlieb, H. E.; Nudelman, A.; Stoltz, B. M.; Bercaw, J. E.; Goldberg, K. I. NMR Chemical Shifts of Trace Impurities: Common 
Laboratory Solvents, Organics, and Gases in Deuterated Solvents Relevant to the Organometallic Chemist. Organometallics 2010, 29, 2176-2179.

42. Schrauzer, G. N.; Sibert, J. W.; Windgassen, R. J. Photochemical and thermal cobaltcarbon bond cleavage in alkylcobalamins and related organometallic compounds. Comparative study. J. Am. Chem. Soc. 1968, 90, 6681-6688.

43. Vaughn, G. D.; Krein, K. A.; Gladysz, J. A. Synthesis and reactivity of metallacyclic $\begin{array}{llll}\text { manganese } & \text { alpha.-(silyloxy)alkyl complexes }\end{array}$ (CO)4MnC(R)(OSi(CH3)3)P(C6H5)2. A new thermodynamic driving force for carbonyl insertion. Organometallics 1986, 5, 936-942.

44. Emslie, D. J. H.; Piers, W. E.; Parvez, M.; McDonald, R. Organometallic Complexes of Scandium and Yttrium Supported by a Bulky Salicylaldimine Ligand. Organometallics 2002, 21, 4226-4240. 
Synthesis, Characterization and Reactivity of Neutral Octahedral Alkyl-Cobalt(III) Complexes Bearing a Dianionic Pendadentate Ligand

Lucie Nurdin, Warren E. Piers, ${ }^{*}$ Jian-Bin Lin and Benjamin S. Gelfand

Department of Chemistry, University of Calgary, 2500 University Drive NW, Calgary, Alberta,

T2N1N4 Canada. *e-mail: wpiers@ucalgary.ca

Supporting Information

Table of Contents

Physical methods, computational details, further synthetic details

Figures S1-S39

S6-S28

NMR spectra of characterized compounds- Figures S40-S59

S29-S45

Additional crystallographic data of complex 4

S46-47

Tables S1 and S2

S48-S49

Cartesian coordinates for calculated structures

S50-S59 


\section{Experimental Details.}

\section{Physical Methods}

${ }^{1} \mathrm{H},{ }^{13} \mathrm{C}\left\{{ }^{1} \mathrm{H}\right\}$ chemical shifts are referenced to the residual solvent signals of $\mathrm{C}_{6} \mathrm{D}_{6}\left({ }^{1} \mathrm{H}, 7.16\right.$ ppm; ${ }^{13} \mathrm{C}\left\{{ }^{1} \mathrm{H}\right\}, 128.06$ ppm), THF-d8 $\left({ }^{1} \mathrm{H}, 3.58,1.72 \mathrm{ppm} ;{ }^{13} \mathrm{C}\left\{{ }^{1} \mathrm{H}\right\}, 67.21,25.31 \mathrm{ppm}\right)$ and toluene-d8 $\left({ }^{1} \mathrm{H}, 7.09,7.01,6.97,2.08 \mathrm{ppm} ;{ }^{13} \mathrm{C}\left\{{ }^{1} \mathrm{H}\right\}, 137.48,128.87,127.96,125.13,20.43\right.$ ppm). ${ }^{1} \mathrm{H},{ }^{11} \mathrm{~B},{ }^{13} \mathrm{C}\left\{{ }^{1} \mathrm{H}\right\},{ }^{19} \mathrm{~F},{ }^{29} \mathrm{Si}\left\{{ }^{1} \mathrm{H}\right\},{ }^{1} \mathrm{H}-{ }^{1} \mathrm{H}-\mathrm{COSY},{ }^{1} \mathrm{H}-{ }^{13} \mathrm{C}-\mathrm{HSQC}$ and ${ }^{1} \mathrm{H}-{ }^{13} \mathrm{C}-\mathrm{HMBC}$ NMR experiments were performed at room temperature on Bruker RDQ-400, or Ascend-500 or Avance-600 MHz spectrometers and analyzed with MestReNova software (v8.1, Mestrelab Research S.L.). All ${ }^{11} \mathrm{~B}$ chemical shifts are relative to $\mathrm{BF}_{3} \cdot \mathrm{OEt}_{2}$, all ${ }^{19} \mathrm{~F}$ chemical shifts are relative to hexafluorobenzene. X-ray crystallographic analyses were performed on suitable crystals coated in Paratone 8277 oil (Exxon) and mounted on a glass loop. Diffraction experiments were performed on a Bruker Smart diffractometer equipped with an Incoatec Microfocus ( $\mathrm{Cu} \mathrm{Ka,} \lambda=1.54178 \AA$ ) and an APEX II CCD detector. The crystals were kept at $173 \mathrm{~K}$ during data collection. Diffractions spots were integrated and scaled with SAINT (Bruker-AXS. SAINT; Madison, Wisconsin, USA, 2017) and the space group was determined with XPREP (Bruker-AXS. XPREP; Madison, Wisconsin, USA, 2017.) Using Olex $2^{1}$, the structures were solved with the ShelX $\mathrm{X}^{2}$ structure solution program using Intrinsic Phasing and refined with the ShelXL ${ }^{3}$ refinement package using Least Squares minimization. Full crystallography details can be found in independently uploaded .cif files. Elemental analysis was performed on site by Johnson Li using a Perkin Elmer Model 2400 series II analyzer. Solution high resolution-mass spectrometry experiments were performed on a Kratos MS-80 spectrometer by Wade White (direct ESI-MS or APCI-MS) on samples prepared in the glovebox in a gas tight syringe. Cyclic voltammetry experiments were carried out using a $\mathrm{CH}$ instrument potentiostat and C-3 cell stand. A glassy carbon working electrode, a platinum counter electrode and a silver wire pseudo reference electrode were used for cyclic experiments in THF with $0.1 \mathrm{M}\left[\mathrm{Bu}_{4} \mathrm{~N}\right]\left[\mathrm{PF}_{6}\right]$ electrolyte in a glovebox. Ferrocene $\left(\mathrm{E}_{\mathrm{Fc}+/ 0}=0.64\right.$ $\checkmark$ vs SHE) was added during each experiment as an internal reference.

\section{Computational Details}

All the quantum-chemical calculations were conducted using the Gaussian16 program suite. ${ }^{4}$ As functional we have used the Becke's 3-parameter hybrid one ${ }^{5}$ combined with the non-

\footnotetext{
${ }^{1}$ Dolomanov, O.V., Bourhis, L.J., Gildea, R.J, Howard, J.A.K. \& Puschmann, H. (2009), J. Appl. Cryst. 42, 339-341.

${ }^{2}$ Sheldrick, G.M. (2015). Acta Cryst. A71, 3-8.

${ }^{3}$ Sheldrick, G.M. (2015). Acta Cryst. C71, 3-8.

${ }^{4}$ Gaussian 16, Revision B.01, M. J. Frisch, G. W. Trucks, H. B. Schlegel, G. E. Scuseria, M. A. Robb, J. R. Cheeseman, G. Scalmani, V. Barone, G. A. Petersson, H. Nakatsuji, X. Li, M. Caricato, A. V. Marenich, J. Bloino, B. G. Janesko, R. Gomperts, B. Mennucci, H. P. Hratchian, J. V. Ortiz, A. F. Izmaylov, J. L. Sonnenberg, D. Williams-Young, F. Ding, F. Lipparini, F. Egidi, J. Goings, B. Peng, A. Petrone, T. Henderson, D. Ranasinghe, V. G. Zakrzewski, J. Gao, N. Rega, G. Zheng, W. Liang,
} 
local correlation functional provided by Perdew/Wang, denoted as B3PW91. ${ }^{6}$ For Co, the relativistic energy-consistent pseudopotential of the Stuttgart-Köln ECP library was used in combination with its adapted segmented basis. ${ }^{7}$ For all other atoms, a standard $6-31 \mathrm{G}^{* *}$ basis set was used. ${ }^{8,9}$ All stationary points have been identified as minima (number of imaginary frequencies Nimag $=0$ ).

\section{Further Synthetic Details.}

\section{Generation of (Cyclopentylmethyl)lithium}

This procedure was done according to a modified literature procedure under $\operatorname{Ar}^{10}$ In a two-neck round bottom flask equipped with a Y-Joint was added iodo(methyl)cyclopentane $(90 \mathrm{mg}, 0.43 \mathrm{mmol})$. A 3:2 ratio of pentane $(500 \mu \mathrm{L})$ and diethyl ether $(340 \mu \mathrm{L})$ was added to the flask to have an overall $0.5 \mathrm{M}$ solution of iodo(methyl)cyclopentane. The solution was cooled to $-78{ }^{\circ} \mathrm{C}$ using an acetone/dry ice bath and ${ }^{\mathrm{t}} \mathrm{BuLi}(1.7 \mathrm{M}, 500 \mu \mathrm{L}, 0.86 \mathrm{mmol})$ was added dropwise under vigorous stirring. The solution was stirred at $-78^{\circ} \mathrm{C}$ for $1 \mathrm{~h}$ and the concentration of $0.32 \mathrm{M}$ was found assuming $100 \%$ yield.

\section{Generation of 5-Hexenyllithium}

This procedure was done according to a modified literature procedure under $\operatorname{Ar}^{10}$

In a two-neck round bottom flask equipped with a Y-Joint was added 6-iodo-1-hexene (90 $\mathrm{mg}, 0.43 \mathrm{mmol})$. A 3:2 ratio of pentane $(500 \mu \mathrm{L})$ and diethyl ether $(340 \mu \mathrm{L})$ was added to the flask to have an overall $0.5 \mathrm{M}$ solution of 6-iodo-1-hexene. The solution was cooled to $-78{ }^{\circ} \mathrm{C}$ using an acetone/dry ice bath and ${ }^{\mathrm{t}} \mathrm{BuLi}(1.7 \mathrm{M}, 500 \mu \mathrm{L}, 0.86 \mathrm{mmol})$ was added dropwise under vigorous stirring. The solution was stirred at $-78^{\circ} \mathrm{C}$ for $1 \mathrm{~h}$ and the concentration of $0.32 \mathrm{M}$ was found assuming $100 \%$ yield.

M. Hada, M. Ehara, K. Toyota, R. Fukuda, J. Hasegawa, M. Ishida, T. Nakajima, Y. Honda, O. Kitao, H. Nakai, T. Vreven, K. Throssell, J. A. Montgomery, Jr., J. E. Peralta, F. Ogliaro, M. J. Bearpark, J. J. Heyd, E. N. Brothers, K. N. Kudin, V. N. Staroverov, T. A. Keith, R. Kobayashi, J. Normand, K. Raghavachari, A. P. Rendell, J. C. Burant, S. S. Iyengar, J. Tomasi, M. Cossi, J. M. Millam, M. Klene, C. Adamo, R. Cammi, J. W. Ochterski, R. L. Martin, K. Morokuma, O. Farkas, J. B. Foresman, and D. J. Fox, Gaussian, Inc., Wallingford CT, 2016.

${ }^{5}$ Becke, A. D. J. Chem. Phys. 1993, 98 (7), 5648.

${ }^{6}$ Perdew, J. P.; Wang, Y. Phys. Rev. B 1992, 45 (23), 13244.

${ }^{7}$ Dolg, M.; Wedig, U.; Stoll, H.; Preuss, H. J. Chem. Phys. 1987, 86 (2), 866.

${ }^{8}$ Hehre, W. J. J. Chem. Phys. 1972, 56 (5), 2257.

${ }^{9}$ Hariharan, P. C.; Pople, J. A. Theor. Chim. Acta 1973, 28 (3), 213.

${ }^{10}$ Bailey, W. F.; Patricia, J. J.; DelGobbo, V. C.; Jarret, R. M.; Okarma, P. J. J. Org. Chem. 1985, 50 (11), 1999. 


\section{Generation of $\mathrm{CpCo(I)} \mathrm{cyclopentadiene} \mathrm{complexes} \mathrm{I-R}$}

Note: $\left[\mathrm{CoCp}_{2}\right]\left[\mathrm{PF}_{6}\right]$ has a very poor solubility in $\mathrm{C}_{6} \mathrm{D}_{6}$. Therefore even though a 1:1 ratio of $\left[\mathrm{CoCp}_{2}\right]\left[\mathrm{PF}_{6}\right]$ and LiR was used, some unreacted LiR remains in solution (See Figures S3031 and S52-57).

Generation of $\mathrm{I}-\mathrm{CH}_{3}$. To a J-Young tube equipped with a Teflon tap, [CoCp $]\left[\mathrm{PF}_{6}\right]$ (30.4 mg, $091 \mathrm{mmol})$ and $\mathrm{LiCH}_{3}(2.0 \mathrm{mg}, 0.091 \mathrm{mmol})$ were added as solids and THF-d8 was added ( $\sim 0.5 \mathrm{~mL})$. Two ${ }^{1} \mathrm{H}$ NMR spectra were taken 30 mins after addition of THF-d8. One spectrum in the diamagnetic region to detect $\mathbf{I}-\mathrm{CH}_{3}$ and one spectrum with a bigger window to detect $\mathrm{CoCp}_{2}$. CoCp 2 , among with ethane and methane were detected (Figures S30-31). ${ }^{1} \mathrm{H}$ NMR (500 MHz, THF-d8) $\delta 5.14(2 \mathrm{H}, \mathrm{m}, \mathrm{H} 4), 4.67(5 \mathrm{H}, \mathrm{s}, \mathrm{H} 1), 2.63(3 \mathrm{H}, \mathrm{m}, \mathrm{H} 3$ \& H2 overlapping), $0.12\left(6 \mathrm{H}, \mathrm{d},{ }^{3} \mathrm{~J}_{\mathrm{HH}}=5.9 \mathrm{~Hz}, \mathrm{H} 5\right) . \mathrm{C}_{2} \mathrm{H}_{6}$ detected at $0.85 \mathrm{ppm} ; \mathrm{CH}_{4}$ detected at $0.19 \mathrm{ppm}$; $\mathrm{CoCp}$ detected at $-51.3 \mathrm{ppm}$.

Note: Due to the very poor solubility of both $\left[\mathrm{CoCp}_{2}\right]\left[\mathrm{PF}_{6}\right]$ and $\mathrm{LiCH}_{3}$ in $\mathrm{C}_{6} \mathrm{D}_{6}$, this reaction was done in THF.

Generation of $\mathrm{I}^{-\mathrm{CH}_{2}} \mathrm{SiMe}_{3}$. To a J-Young tube equipped with a Teflon tap, [CoCp $]$ [PF 6 (25.0 mg, $075 \mathrm{mmol})$ and $\mathrm{LiCH}_{2} \mathrm{SiMe}_{3}(7.0 \mathrm{mg}, 0.075 \mathrm{mmol})$ were added as solids and $\mathrm{C}_{6} \mathrm{D}_{6}$ was added $(\sim 0.5 \mathrm{~mL})$. Two ${ }^{1} \mathrm{H}$ NMR spectra were taken 30 mins after addition of $\mathrm{C}_{6} \mathrm{D}_{6}$. One spectrum in the diamagnetic region to detect $\mathbf{I}-\mathrm{CH}_{2} \mathrm{SiMe}_{3}$ and one spectrum with a bigger window to detect $\mathrm{CoCp}_{2}$ (Figures S52-53). No $\mathrm{CoCp}_{2}$ was detected. ${ }^{1} \mathrm{H}$ NMR $(500 \mathrm{MHz}$, $\left.\mathrm{C}_{6} \mathrm{D}_{6}\right) \delta 5.02(2 \mathrm{H}, \mathrm{m}, \mathrm{H} 4), 4.60(5 \mathrm{H}, \mathrm{s}, \mathrm{H} 1), 2.73$ (3H, m, H3 \& H2 overlapping), 0.00 (2H, br, $\mathrm{H} 5),-0.02(9 \mathrm{H}, \mathrm{s}, \mathrm{H} 6)$.

Generation of I-CH $\mathrm{CH}_{2} \mathrm{SiMe}_{2} \mathrm{Ph}$. To a J-Young tube equipped with a Teflon tap, [CoCp $\left.{ }_{2}\right]\left[\mathrm{PF}_{6}\right]$ (20.0 mg, $0.060 \mathrm{mmol}$ ) and $\mathrm{LiCH}_{2} \mathrm{SiMe}_{2} \mathrm{Ph}(9.4 \mathrm{mg}, 0.060 \mathrm{mmol})$ were added as solids and then $\mathrm{C}_{6} \mathrm{D}_{6}$ was added ( $\left.\sim 5 \mathrm{~mL}\right)$. Two ${ }^{1} \mathrm{H}$ NMR spectra were taken 30 mins after addition of $\mathrm{C}_{6} \mathrm{D}_{6}$. One spectrum in the diamagnetic region to detect $\mathbf{I}-\mathrm{CH}_{2} \mathbf{S i M e} \mathbf{P}_{2} \mathbf{P h}$ and one spectrum with a bigger window to detect $\mathrm{CoCp}_{2}$ (Figures S54-55). No CoCp 2 was detected. ${ }^{1} \mathrm{H}$ NMR $\left(500 \mathrm{MHz}, \mathrm{C}_{6} \mathrm{D}_{6}\right) \delta 7.44(2 \mathrm{H}, \mathrm{m}, \mathrm{H} 7), 7.20(3 \mathrm{H}, \mathrm{m}, \mathrm{H} 8$ \& H9), $4.98(2 \mathrm{H}, \mathrm{m}, \mathrm{H} 4), 4.54(5 \mathrm{H}, \mathrm{s}$, $\mathrm{H} 1), 2.76(1 \mathrm{H}, \mathrm{m}, \mathrm{H} 2), 2.66(2 \mathrm{H}, \mathrm{m}, \mathrm{H} 3), 0.30\left(2 \mathrm{H}, \mathrm{d},{ }^{3} \mathrm{~J}_{\mathrm{HH}}=6.7 \mathrm{~Hz}, \mathrm{H} 5\right), 0.20(6 \mathrm{H}, \mathrm{s}, \mathrm{H} 6)$.

Generation of $\mathrm{I}-\mathrm{CH}_{2} \mathbf{C H}\left(\mathrm{CH}_{3}\right)_{2}$. To a J-Young tube equipped with a Teflon tap, [CoCp $\left.\mathrm{Co}_{2}\right]\left[\mathrm{PF}_{6}\right]$ $(20.0 \mathrm{mg}, 0.060 \mathrm{mmol})$ and Li'Bu $(3.8 \mathrm{mg}, 0.060 \mathrm{mmol})$ were added as solids and $\mathrm{C}_{6} \mathrm{D}_{6}$ was added $(\sim 0.5 \mathrm{~mL})$. Two ${ }^{1} \mathrm{H}$ NMR spectra were taken 30 mins after addition of $\mathrm{C}_{6} \mathrm{D}_{6}$. One spectrum in the diamagnetic region to detect I-iBu and one spectrum with a bigger window to detect $\mathrm{CoCp}_{2}$ (Figures S56-57). No CoCp 2 was detected. ${ }^{1} \mathrm{H} \mathrm{NMR}\left(500 \mathrm{MHz}, \mathrm{C}_{6} \mathrm{D}_{6}\right) \delta 5.04$ $(2 \mathrm{H}, \mathrm{dd}, \mathrm{J}=2.5,1.5 \mathrm{~Hz}, \mathrm{H} 4), 4.60(5 \mathrm{H}, \mathrm{s}, \mathrm{H} 1), 2.67(3 \mathrm{H}, \mathrm{m}, \mathrm{H} 3$ \& H2 overlapping), $1.42(1 \mathrm{H}$, $\mathrm{m}, \mathrm{H} 6), 0.78\left(6 \mathrm{H}, \mathrm{d},{ }^{3} \mathrm{~J}_{\mathrm{HH}}=6.6 \mathrm{~Hz}, \mathrm{H} 7\right), 0.48(2 \mathrm{H}, \mathrm{dd}, \mathrm{J}=6.6 \mathrm{~Hz}, \mathrm{H} 5)$. 
Numbering schemes for ${ }^{1} \mathrm{H}$ NMR assignments for compounds I-R:
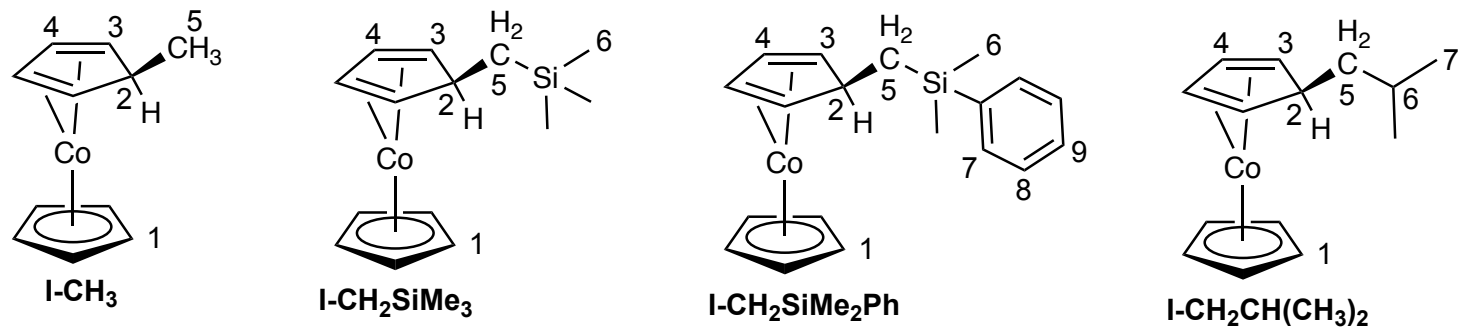
Figures S1-S39:

Procedure for the reactivity of $2-R$ in the presence of 1,4-cyclohexadiene (CHD) in Toluene-d8.

A J-Young tube equipped with a Teflon tap was charged with $5.0 \mathrm{mg}$ of $\mathbf{2 - R}$ in toluene-d8. A ${ }^{1} \mathrm{H}$ NMR spectrum was recorded. CHD (5 eq) was added at room temperature and another ${ }^{1} \mathrm{H}$ NMR spectrum was recorded. The $\mathrm{Y}$-Young tube was then heated at $80{ }^{\circ} \mathrm{C}$ for $24 \mathrm{~h}$ and another ${ }^{1} \mathrm{H}$ NMR spectrum was recorded. If any reactivity was observed, a ${ }^{11} \mathrm{~B} N M R$ spectrum was recorded to detect complex 3.

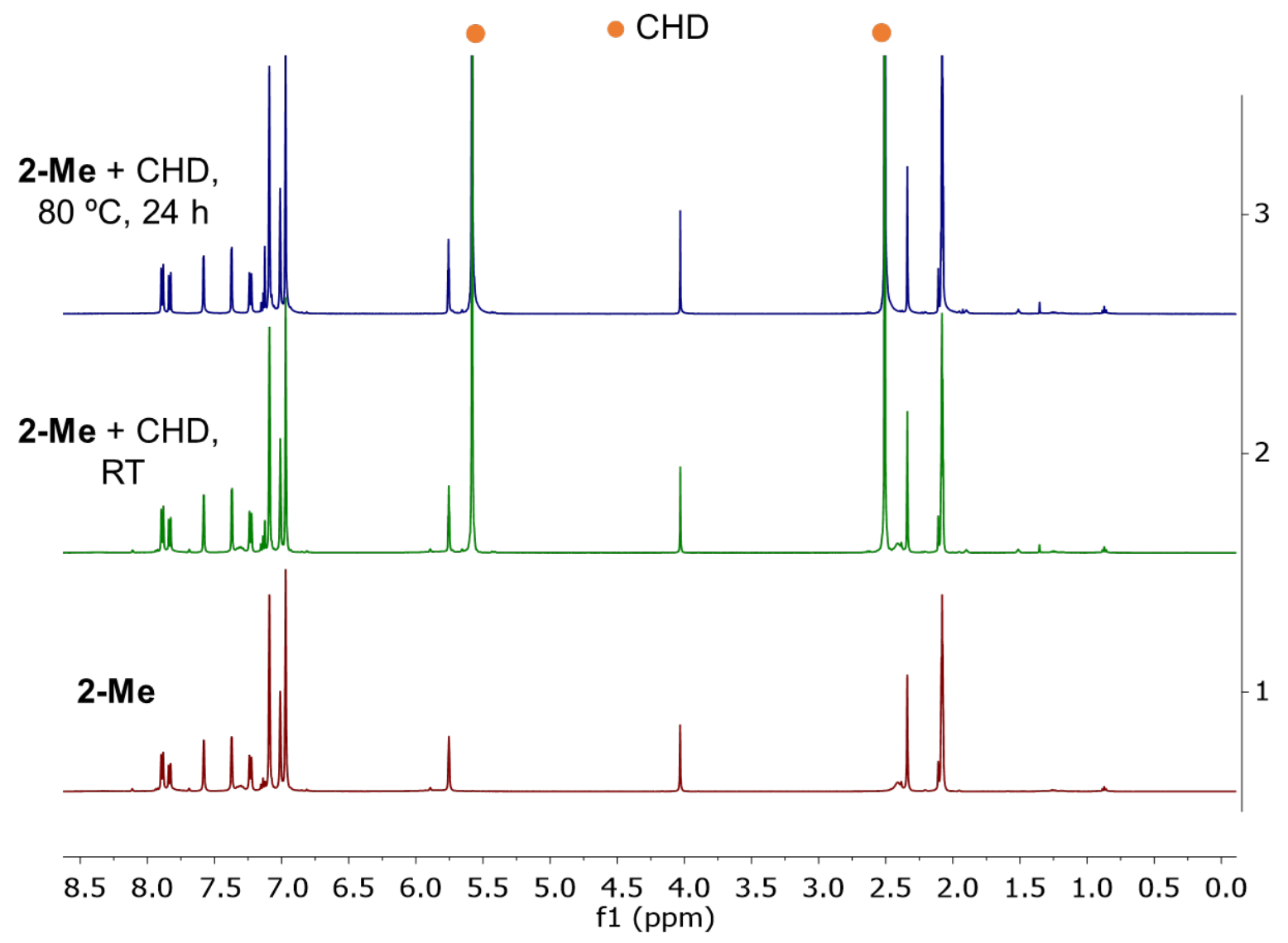

Figure S1. ${ }^{1} \mathrm{H}$ stack NMR spectra of 2-Me (spectrum 1), 2-Me + CHD at RT (spectrum 2) and 2-Me + CHD at $80^{\circ} \mathrm{C}$ (spectrum 3 ) in toluene-d8. 


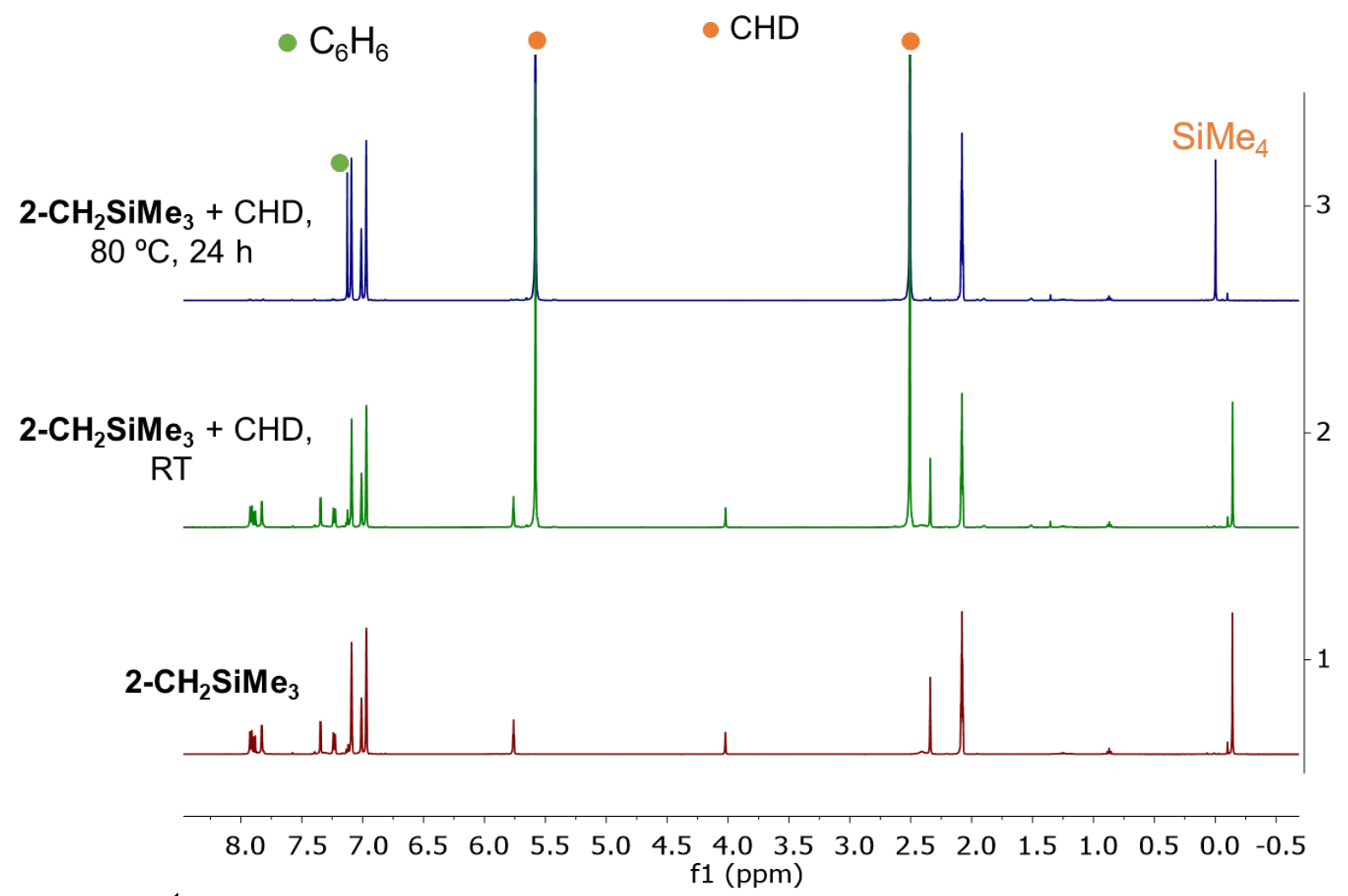

Figure S2. ${ }^{1} \mathrm{H}$ stack NMR spectra of $2-\mathrm{CH}_{2} \mathrm{SiMe}_{3}$ (spectrum 1), $\mathbf{2}-\mathrm{CH}_{2} \mathrm{SiMe}_{3}+\mathrm{CHD}$ at RT (spectrum 2) and $2-\mathrm{CH}_{2} \mathrm{SiMe}_{3}+\mathrm{CHD}$ at $80^{\circ} \mathrm{C}$ (spectrum 3) in toluene-d8. Protio benzene and $\mathrm{SiMe}_{4}$ are detected in spectrum 3.
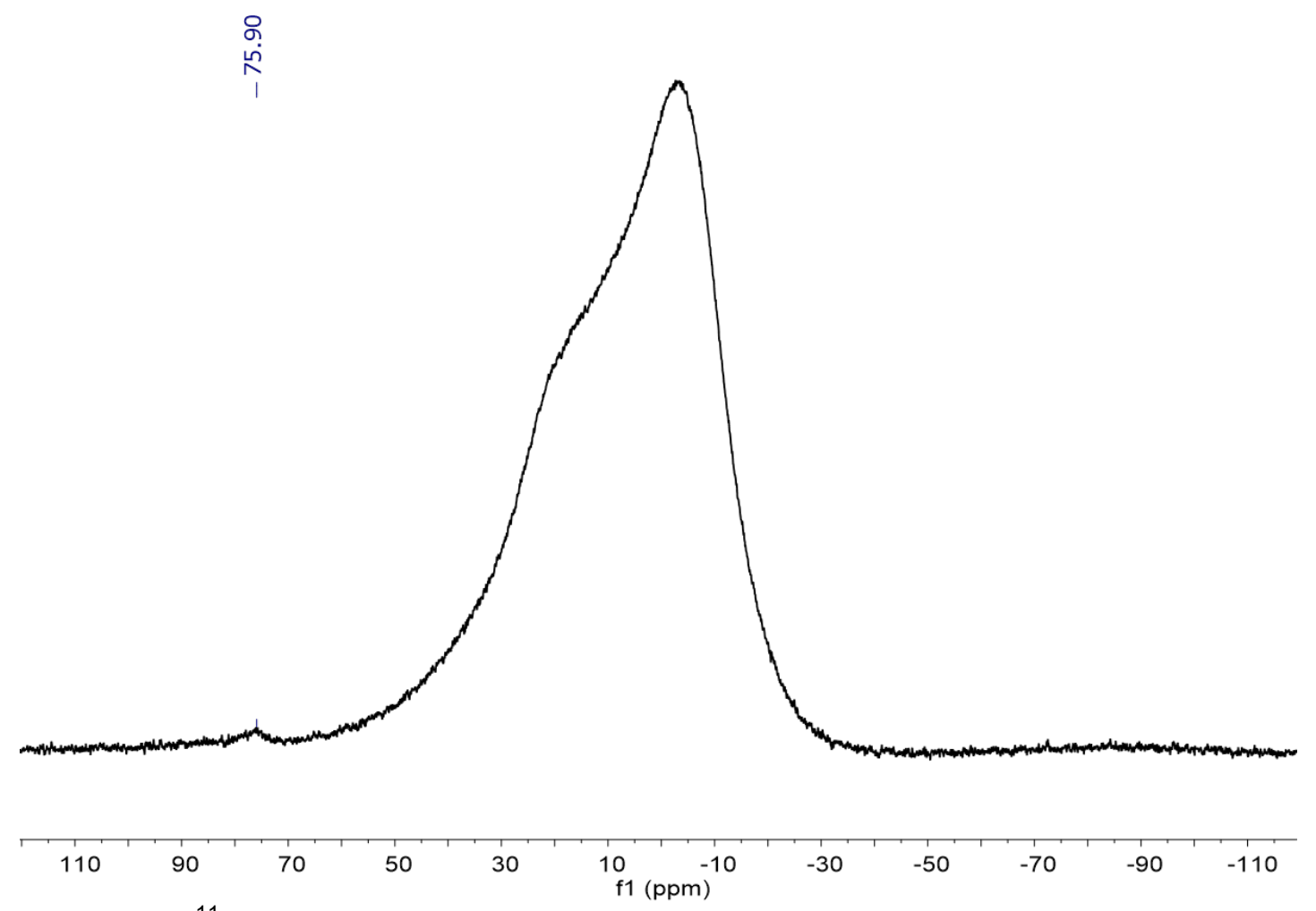

Figure S3. ${ }^{11} \mathrm{~B}$ NMR spectrum of $2-\mathrm{CH}_{2} \mathrm{SiMe}_{3}+\mathrm{CHD}$ at $80{ }^{\circ} \mathrm{C}$ in toluene-d8. The broad peak corresponds to complex 3. 


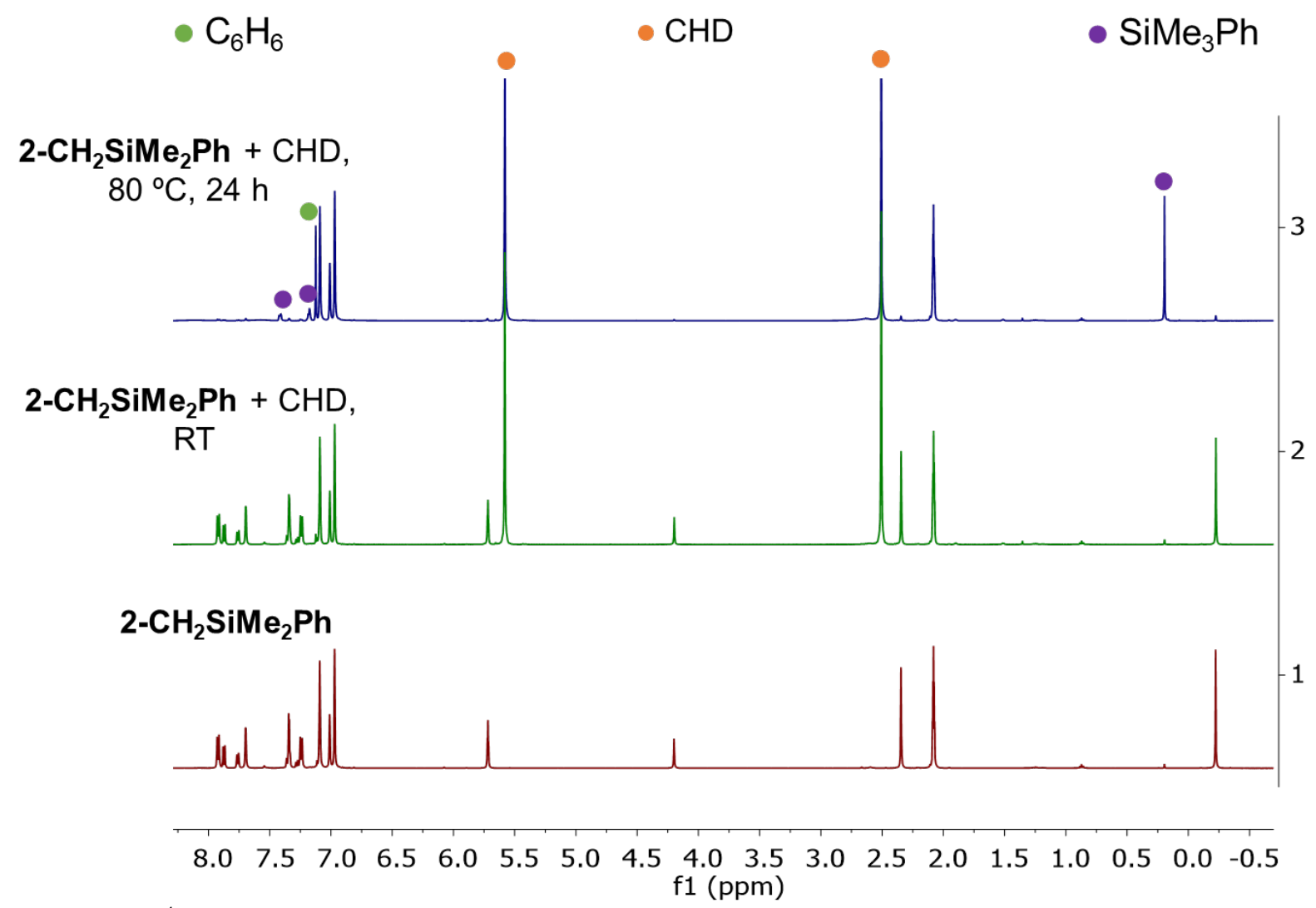

Figure S4. ${ }^{1} \mathrm{H}$ stack NMR spectra of $2-\mathrm{CH}_{2} \mathrm{SiMe}_{2} \mathrm{Ph}$ (spectrum 1), $2-\mathrm{CH}_{2} \mathrm{SiMe}_{2} \mathrm{Ph}+\mathrm{CHD}$ at RT (spectrum 2) and $2-\mathrm{CH}_{2} \mathrm{SiMe}_{2} \mathrm{Ph}+\mathrm{CHD}$ at $80^{\circ} \mathrm{C}$ (spectrum 3) in toluene-d8. Protio benzene and $\mathrm{SiMe}_{3} \mathrm{Ph}$ are detected in spectrum 3.

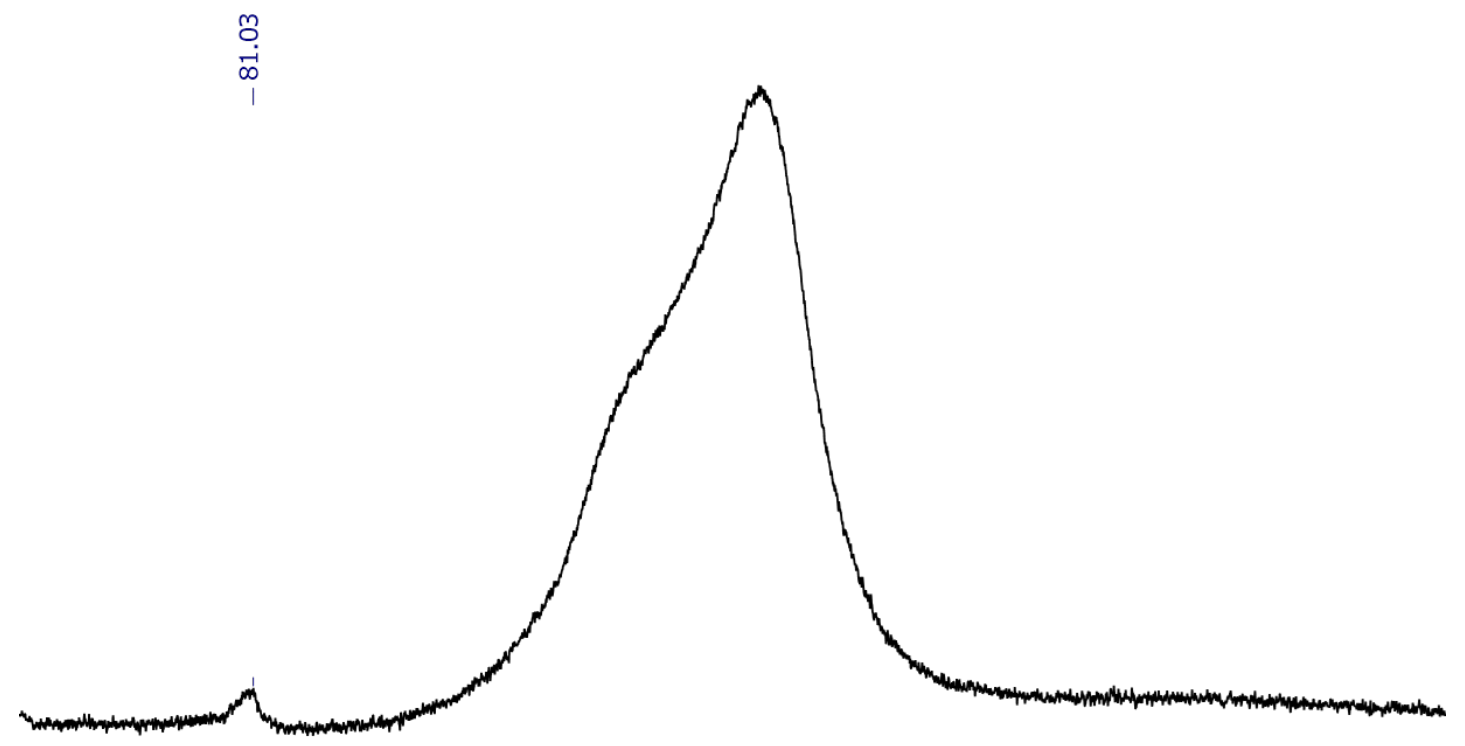

$\begin{array}{lllllllllll}110 & 90 & 70 & 50 & 30 & \begin{array}{l}10 \\ \mathrm{f} 1(\mathrm{ppm})\end{array} & -30 & -50 & -70 & -90 & -110\end{array}$

Figure S5. ${ }^{11} \mathrm{~B}$ NMR spectrum of $2-\mathrm{CH}_{2} \mathrm{SiMe}_{2} \mathrm{Ph}+\mathrm{CHD}$ at $80{ }^{\circ} \mathrm{C}$ in toluene-d8. The broad peak corresponds to complex 3. 


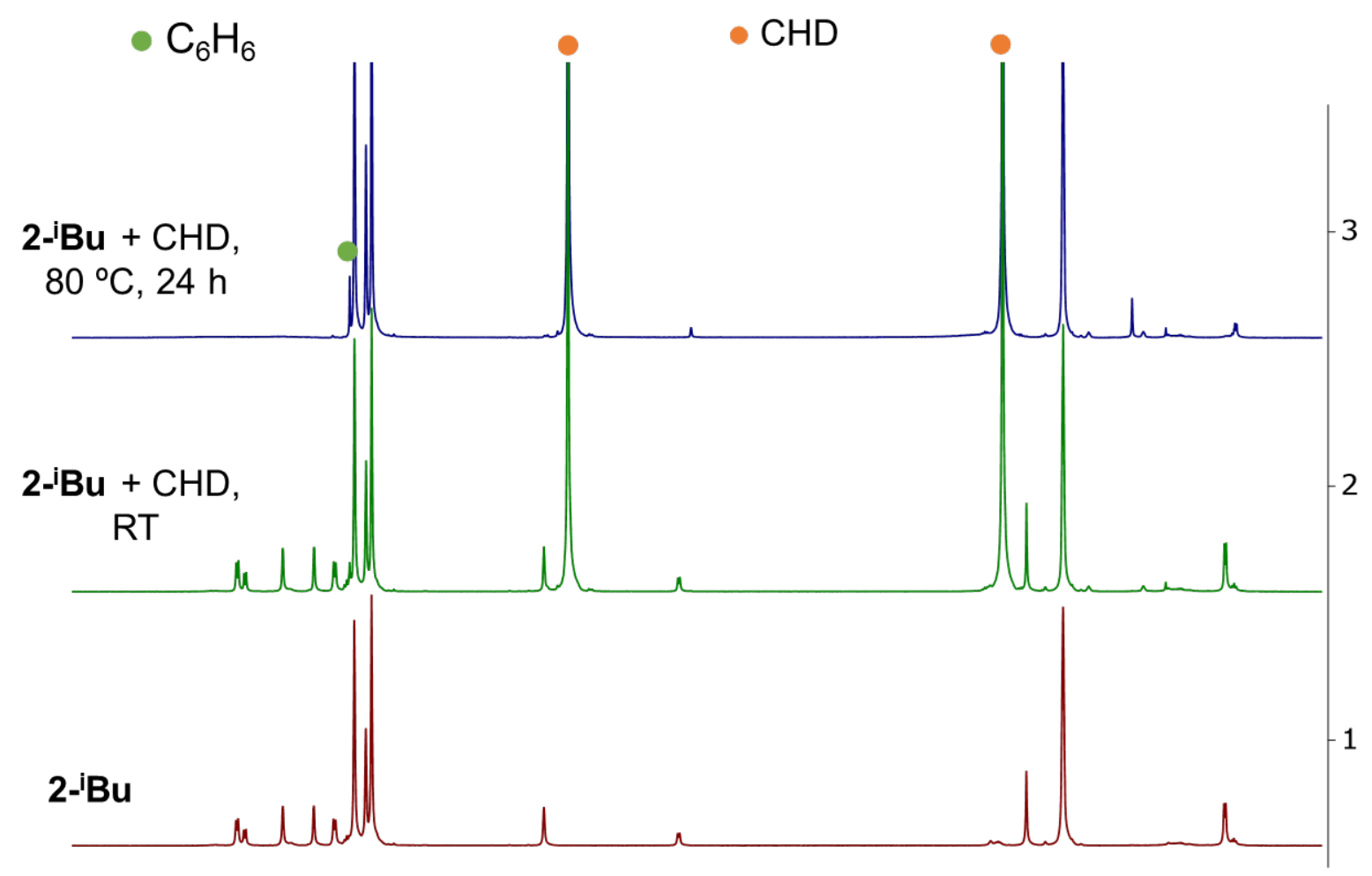

$\begin{array}{llllllllllllllllll}7.0 & 8.5 & 8.0 & 7.5 & 7.0 & 6.5 & 6.0 & 5.5 & 5.0 & 4.5 & 4.0 & 3.5 & 3.0 & 2.5 & 2.0 & 1.5 & 1.0 & 0.5\end{array}$ f1 (ppm)

Figure S6. ${ }^{1} \mathrm{H}$ stack NMR spectra of 2-'Bu (spectrum 2), 2-'Bu + CHD at RT (spectrum 2) and 2-'Bu $+\mathrm{CHD}$ at $80^{\circ} \mathrm{C}$ (spectrum 3) in toluene-d8. Protio benzene is detected in spectrum 3 . $\underset{\substack{q \\ i}}{i}$

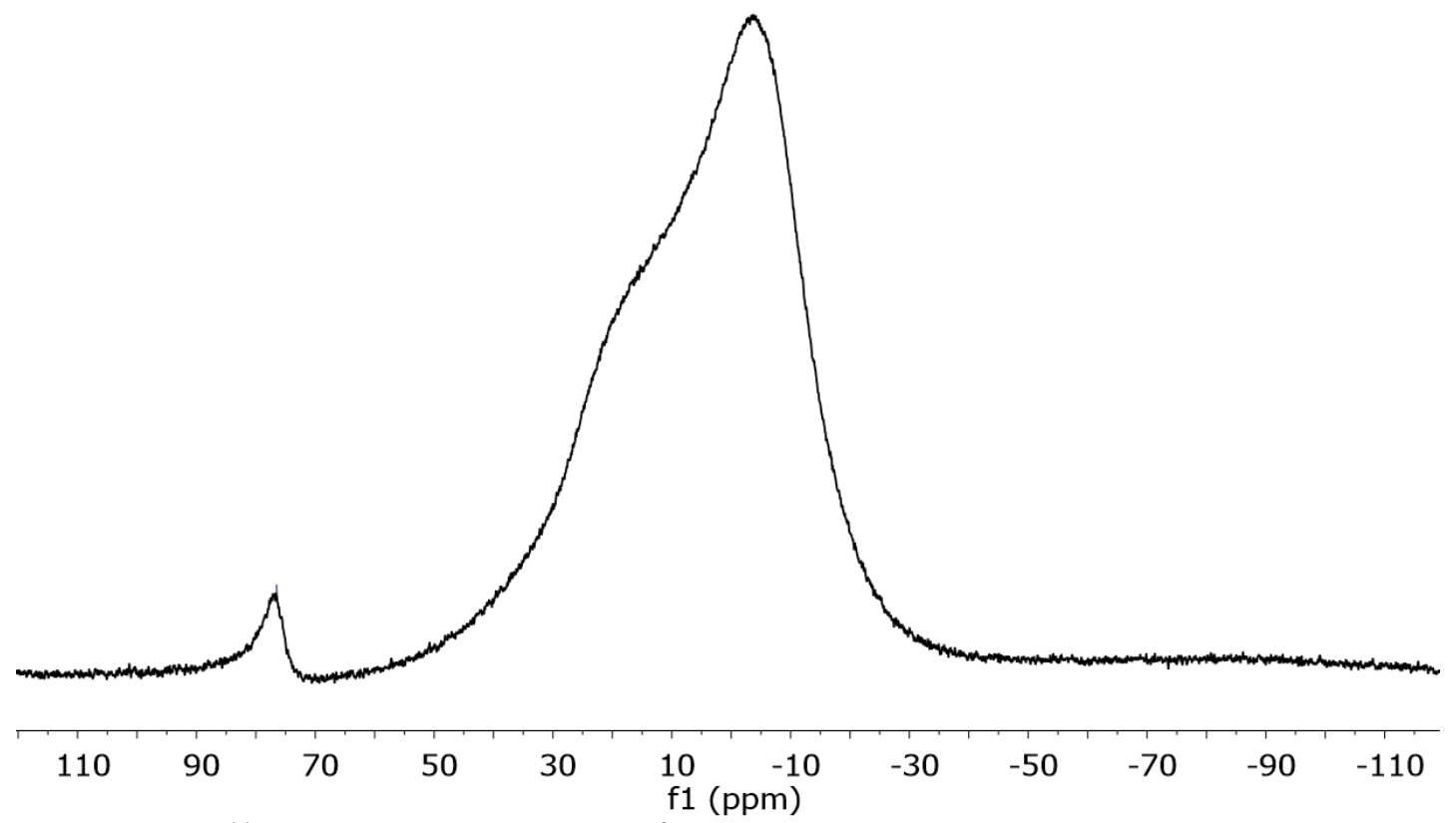

Figure S7. ${ }^{11} \mathrm{~B}$ NMR spectrum of $2-\mathrm{Bu}+\mathrm{CHD}$ at $80^{\circ} \mathrm{C}$ in toluene-d8. The broad peak corresponds to complex 3. 


\section{Procedure for the reactivity of $2-R$ with TEMPO in $C_{6} D_{6}$.}

A J-Young tube equipped with a Teflon tap was charged with $5.0 \mathrm{mg}$ of $\mathbf{2}-\mathbf{R}$ in $\mathrm{C}_{6} \mathrm{D}_{6} . \mathrm{A}^{1} \mathrm{H}$ NMR spectrum was recorded. TEMPO (1.1 eq) was added as a solid at room temperature and another ${ }^{1} \mathrm{H}$ NMR spectrum was recorded after $24 \mathrm{~h}$. If any reactivity was observed, a ${ }^{11} \mathrm{~B}$ NMR spectrum was recorded to detect complex 3.

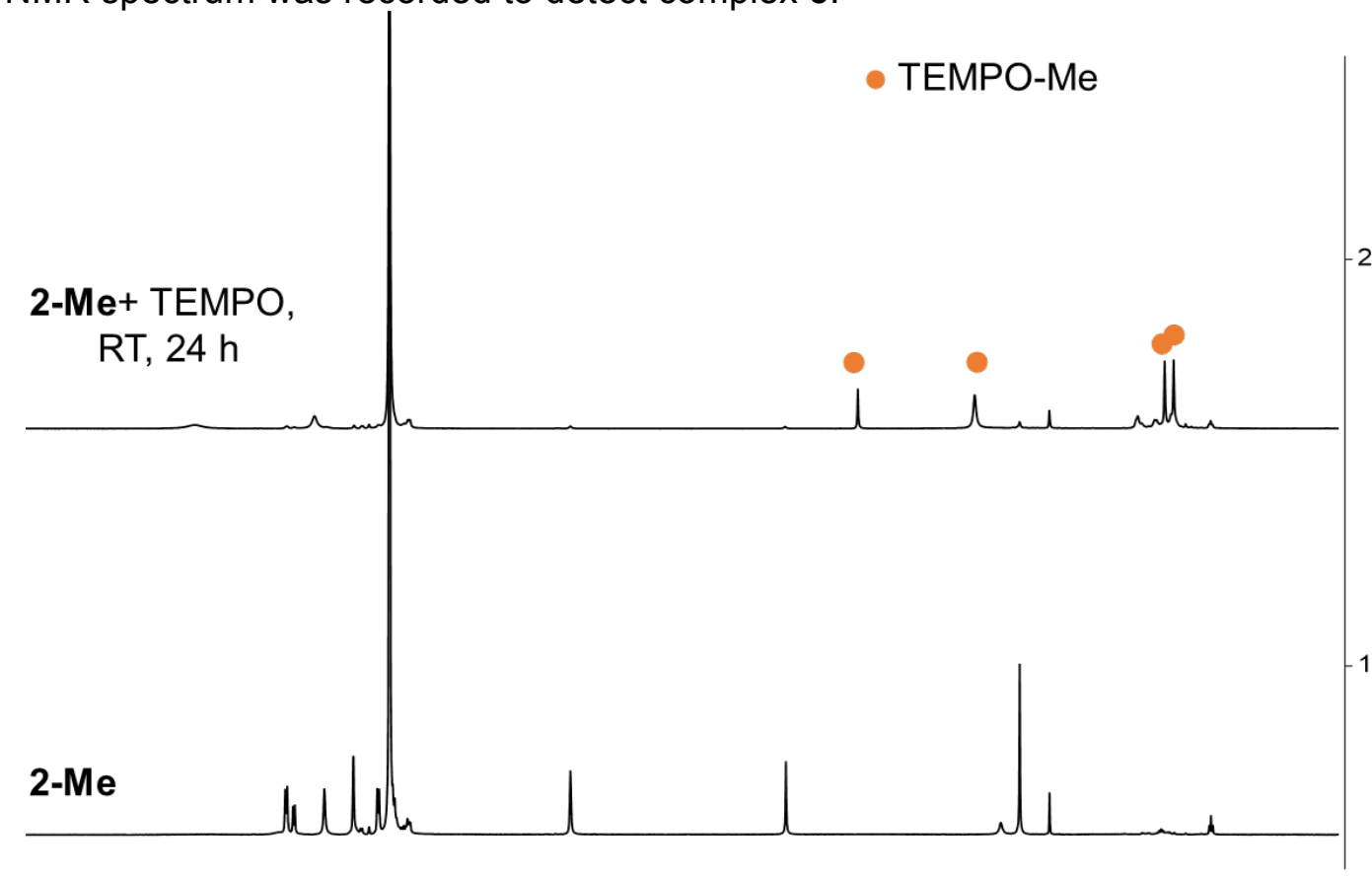

$\begin{array}{lllllllllllllllllllll}9.5 & 9.0 & 8.5 & 8.0 & 7.5 & 7.0 & 6.5 & 6.0 & 5.5 \underset{\mathrm{f} 1}{5.0}(\mathrm{ppm}) & 4.5 & 4.0 & 3.5 & 3.0 & 2.5 & 2.0 & 1.5 & 1.0 & 0.5 & 0.0\end{array}$

Figure S8. ${ }^{1} \mathrm{H}$ stack NMR spectra of 2-Me (spectrum 1) and 2-Me + TEMPO (spectrum 2) in $\mathrm{C}_{6} \mathrm{D}_{6}$.

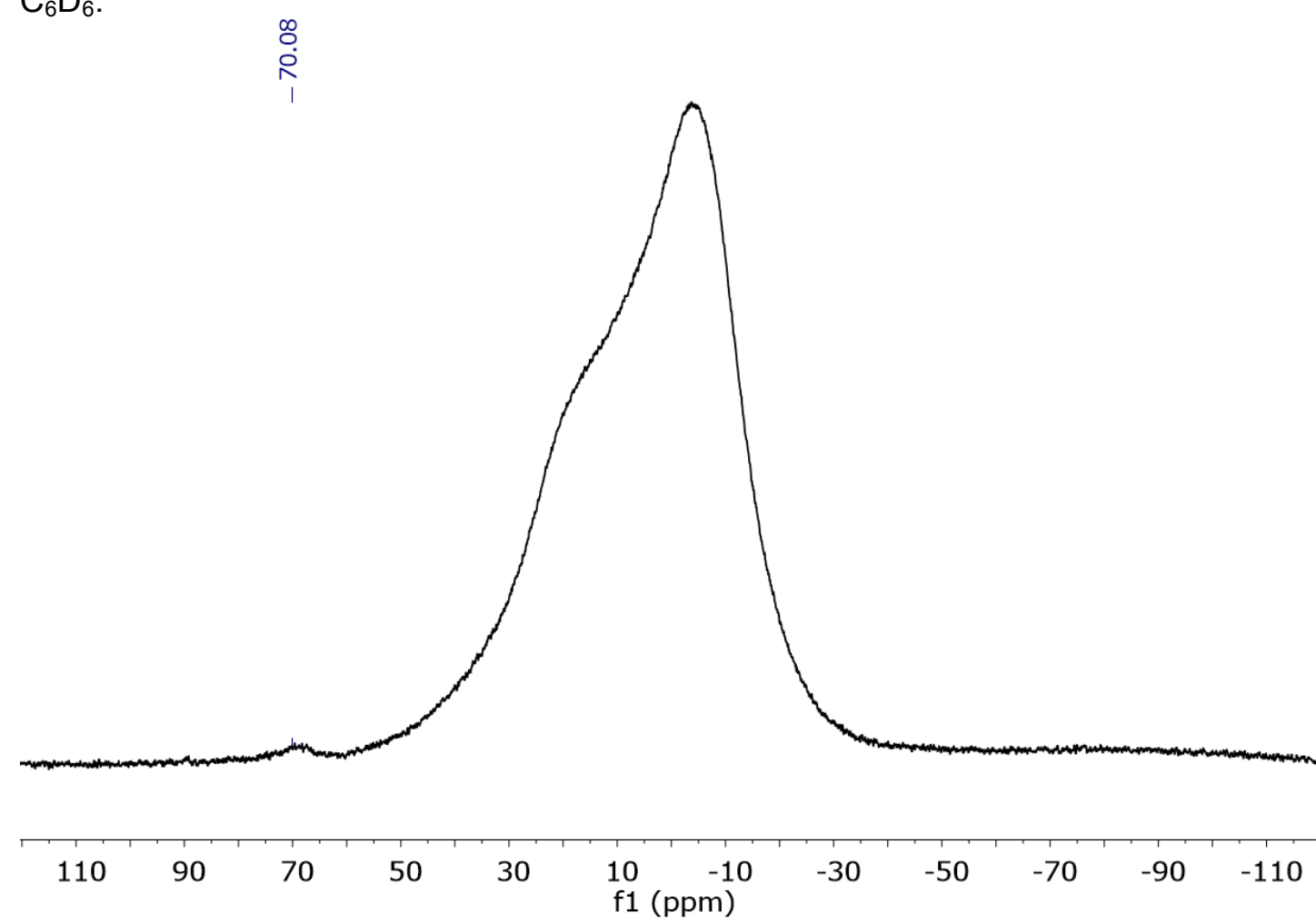

Figure S9. ${ }^{11} \mathrm{~B}$ NMR spectrum of 2-Me + TEMPO in $\mathrm{C}_{6} \mathrm{D}_{6}$. 


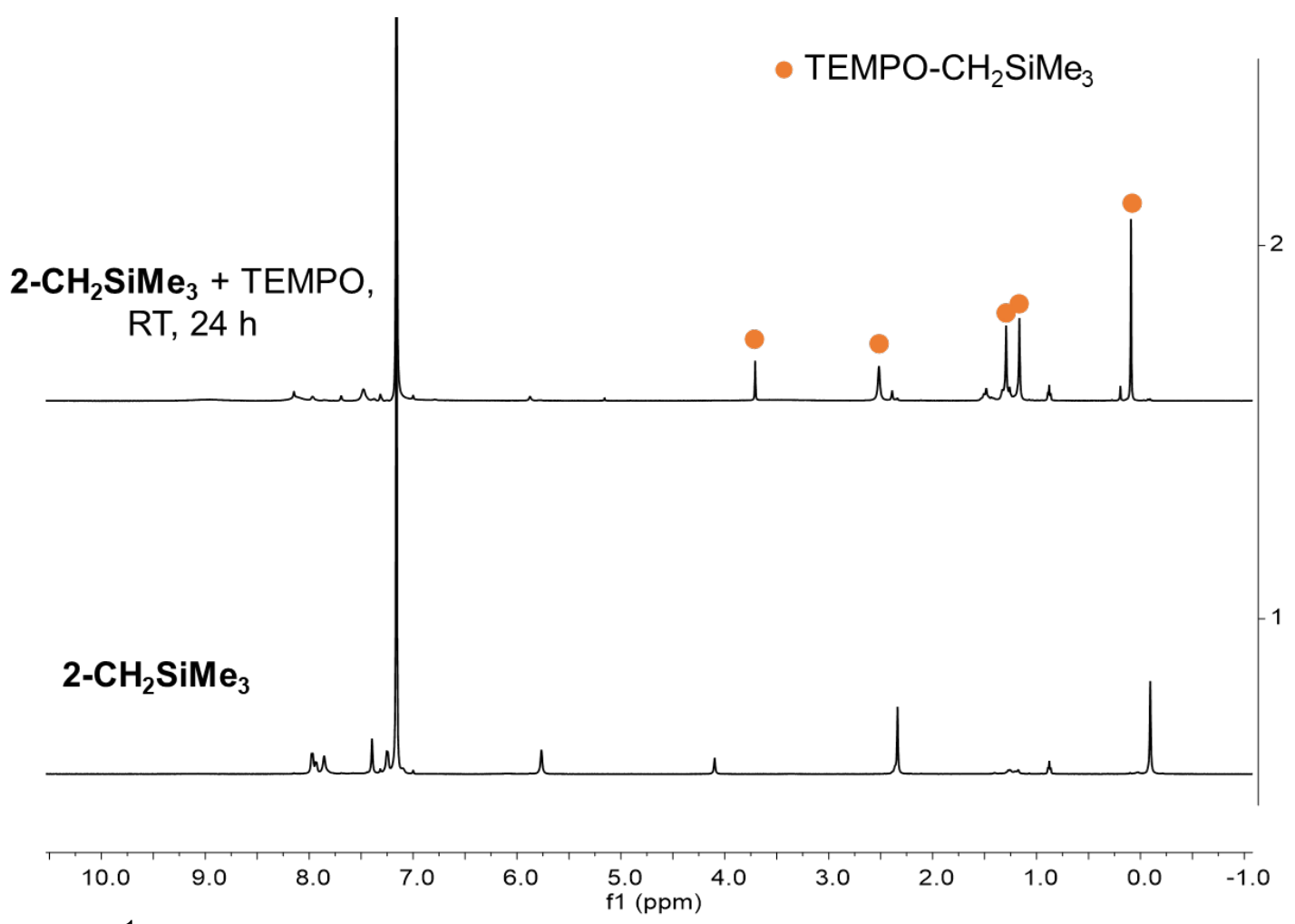

Figure S10. ${ }^{1} \mathrm{H}$ stack NMR spectra of $2-\mathrm{CH}_{2} \mathrm{SiMe}_{3}$ (spectrum 1) and $2-\mathrm{CH}_{2} \mathrm{SiMe}_{3}+$ TEMPO (spectrum 2) in $\mathrm{C}_{6} \mathrm{D}_{6}$.

$\stackrel{m}{\stackrel{m}{n}}$

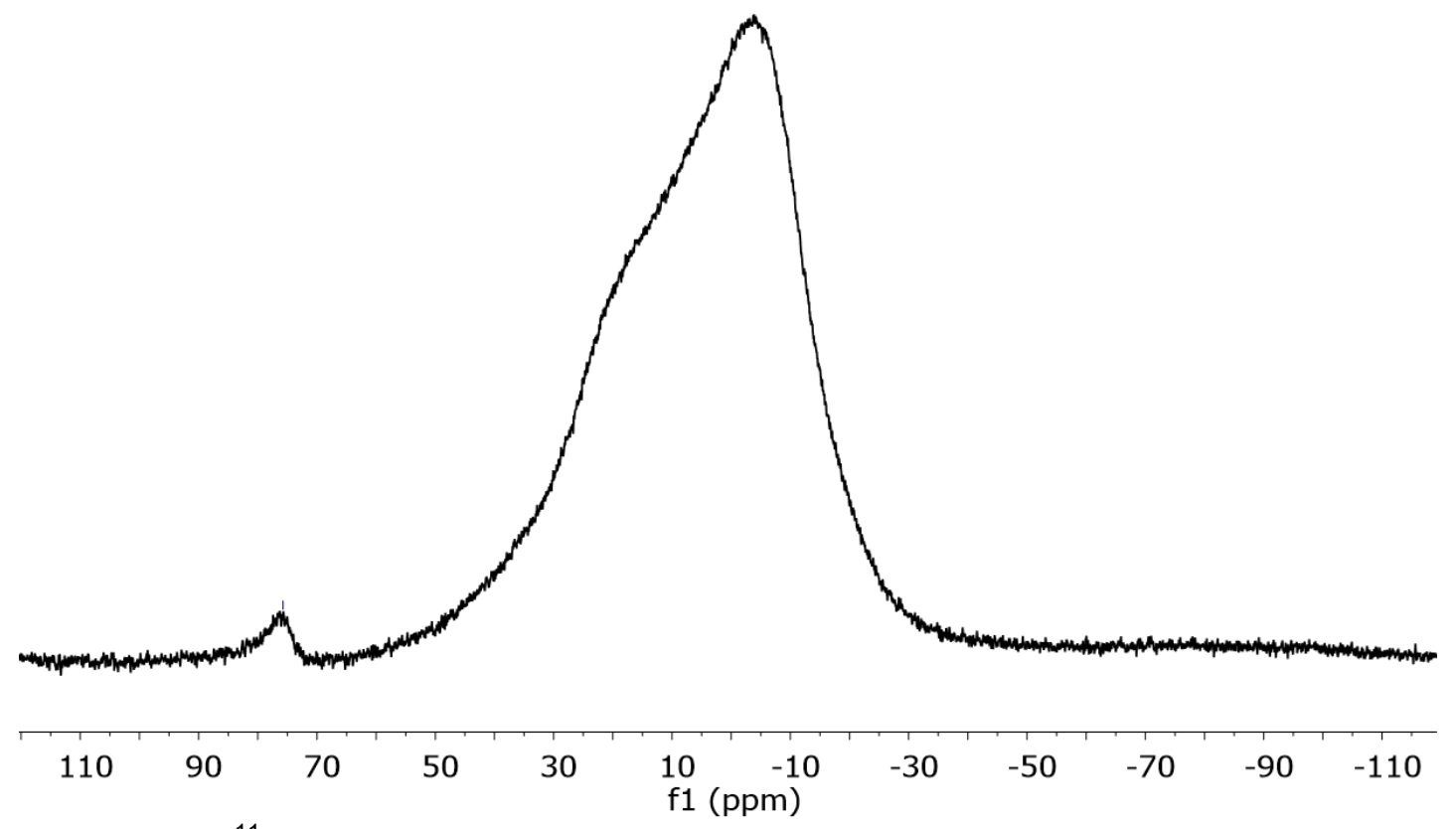

Figure S11. ${ }^{11} \mathrm{~B}$ NMR spectrum of $2-\mathrm{CH}_{2} \mathrm{SiMe}_{3}+\mathrm{TEMPO}$ in $\mathrm{C}_{6} \mathrm{D}_{6}$. 


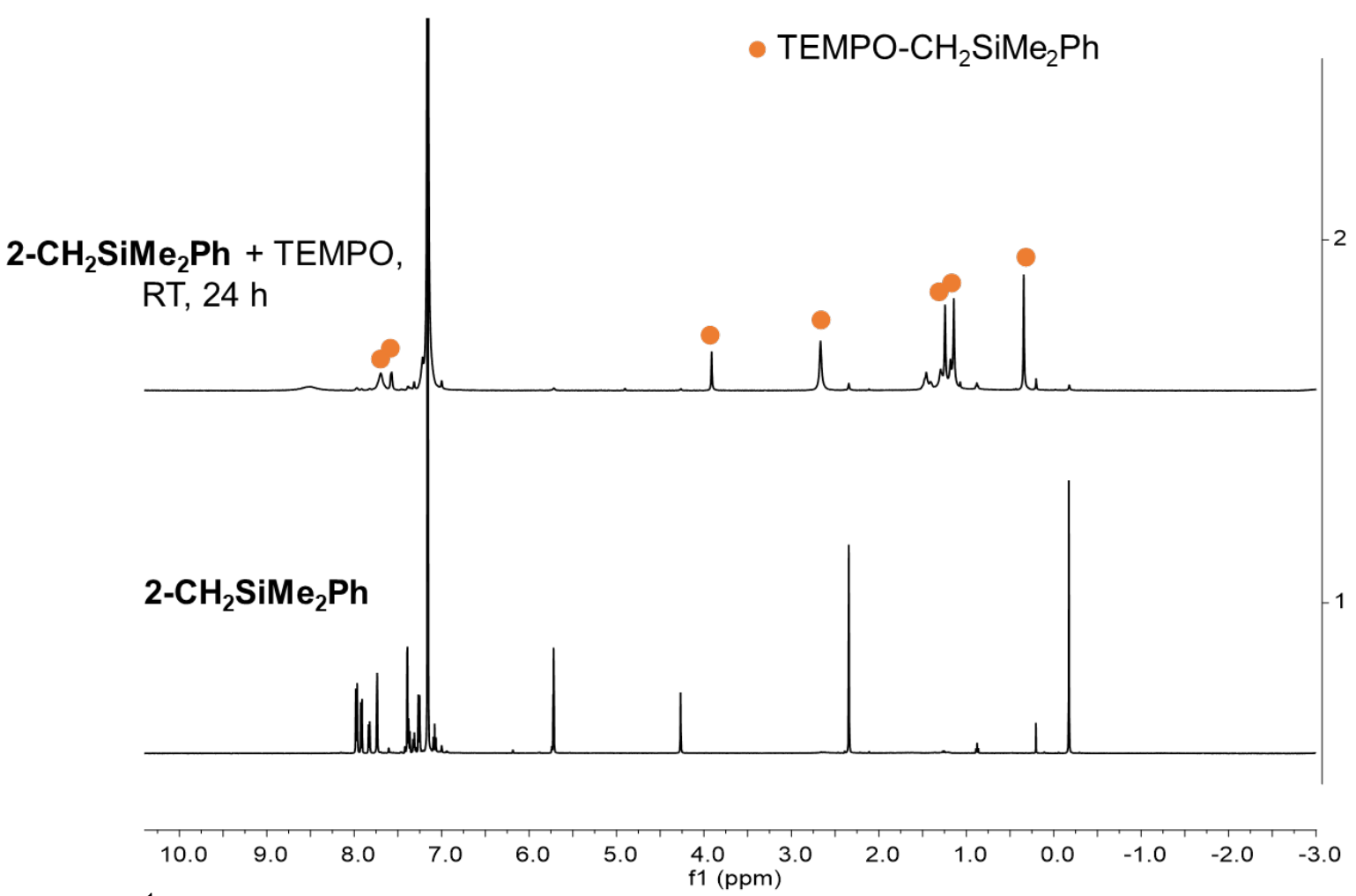

Figure S12. ${ }^{1} \mathrm{H}$ stack NMR spectra of $2-\mathrm{CH}_{2} \mathrm{SiMe}_{2} \mathrm{Ph}$ (spectrum 1) and 2- $\mathrm{CH}_{2} \mathbf{S i M e}{ }_{2} \mathbf{P h}+$ TEMPO (spectrum 2) in $\mathrm{C}_{6} \mathrm{D}_{6}$.

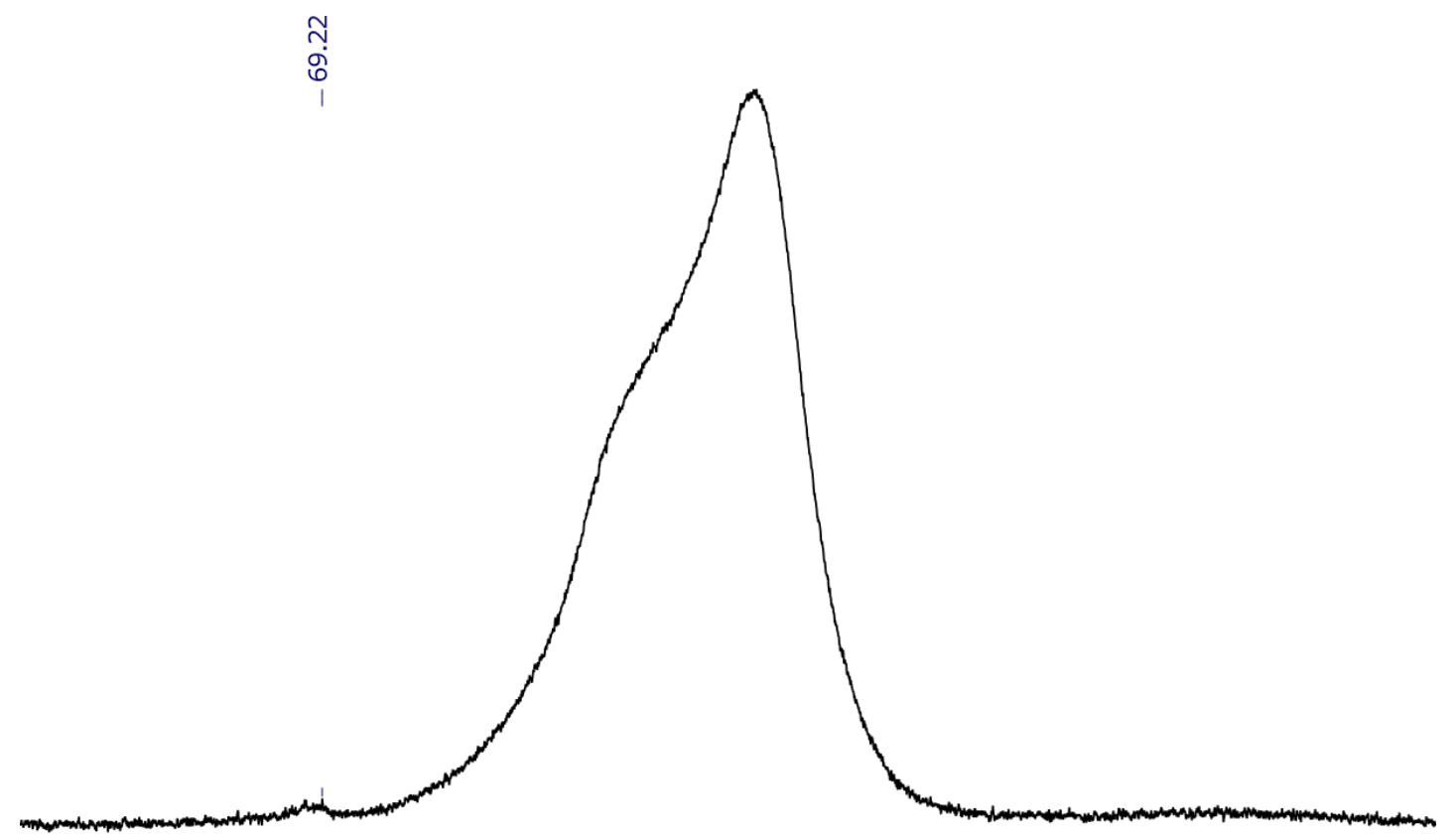

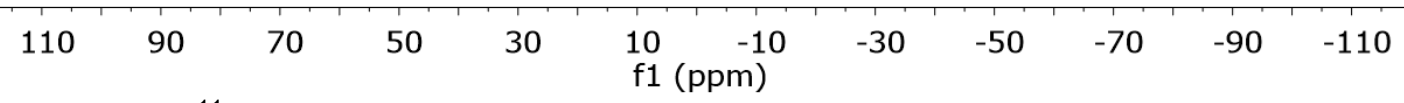

Figure S13. ${ }^{11} \mathrm{~B}$ NMR spectrum of 2- $\mathrm{CH}_{2} \mathrm{SiMe}{ }_{2} \mathrm{Ph}+\mathrm{TEMPO}$ in $\mathrm{C}_{6} \mathrm{D}_{6}$. 


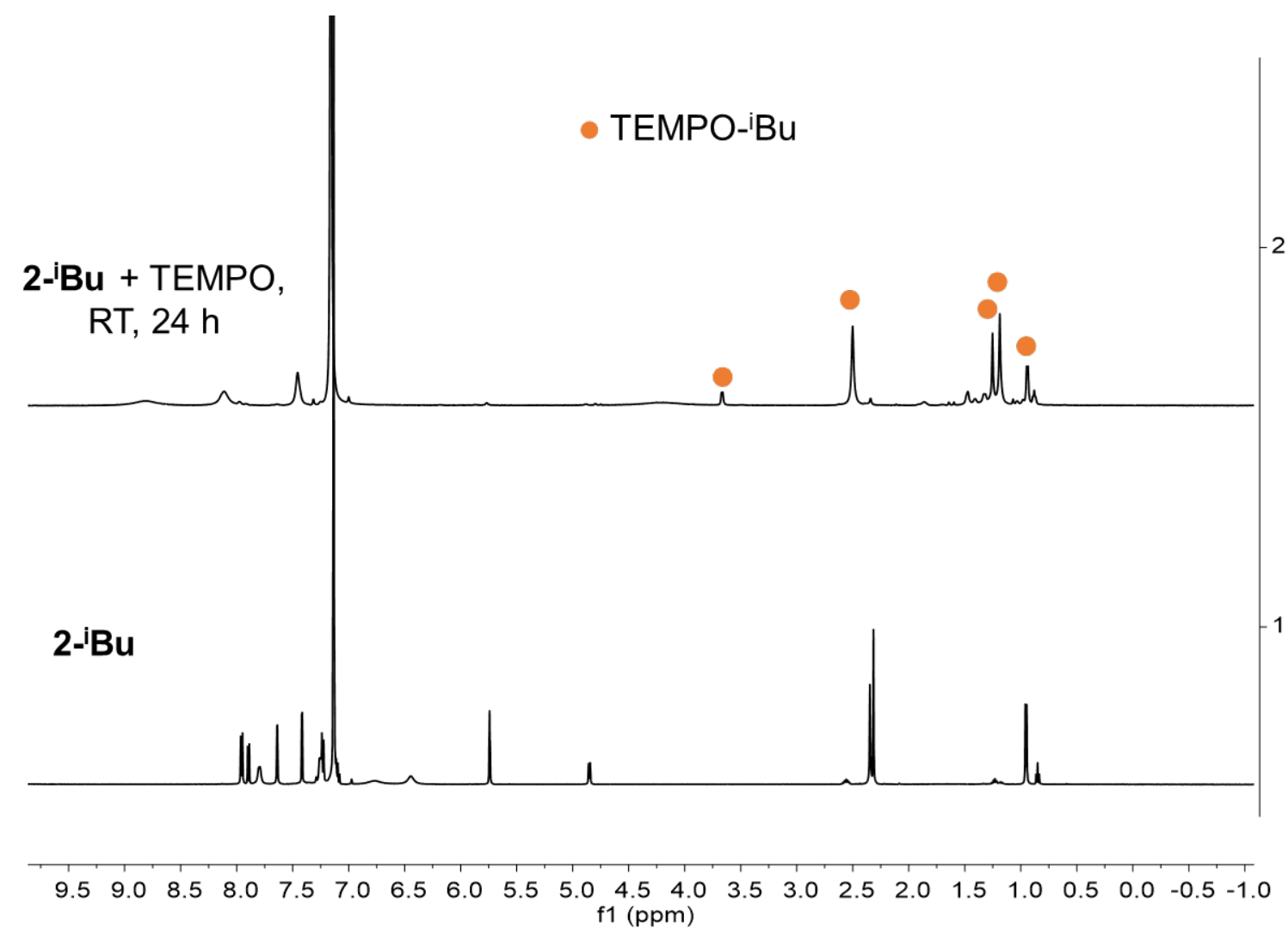

Figure S14. ${ }^{1} \mathrm{H}$ stack NMR spectra of $2-^{\mathrm{i}} \mathrm{Bu}$ (spectrum 1) and 2-'Bu + TEMPO (spectrum 2) in $\mathrm{C}_{6} \mathrm{D}_{6}$.

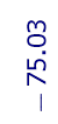

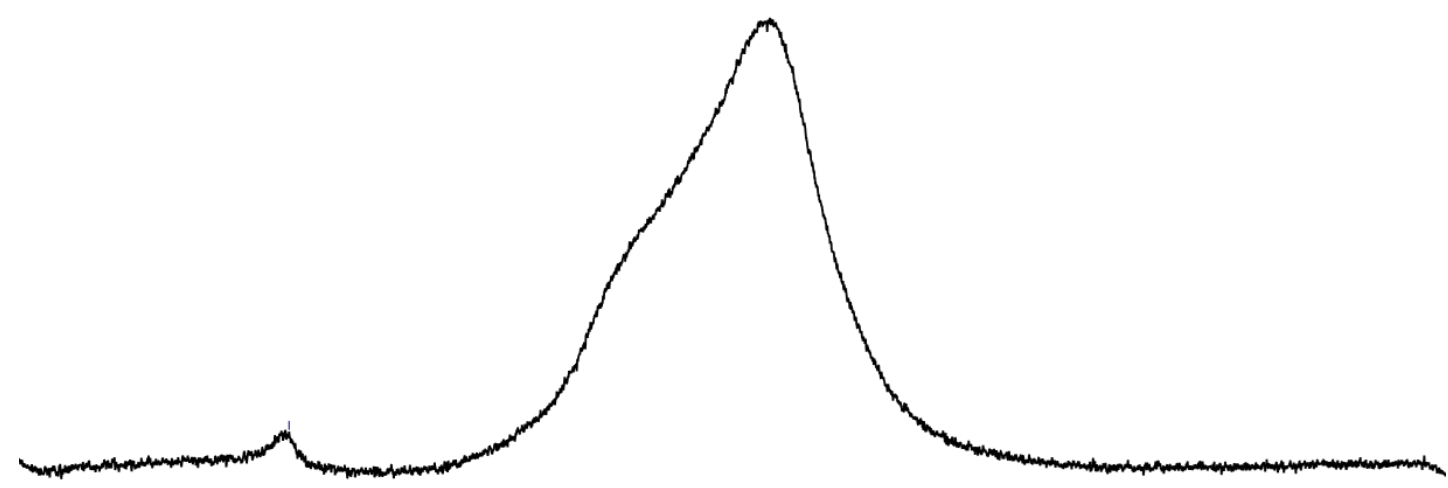

$\begin{array}{lllllllllll}110 & 90 & 70 & 50 & 30 & \begin{array}{c}10 \\ \mathrm{f} 1(\mathrm{ppm})\end{array} & -30 & -50 & -70 & -90 & -110\end{array}$

Figure S15. ${ }^{11} \mathrm{~B}$ NMR spectrum of 2-'Bu + TEMPO in $\mathrm{C}_{6} \mathrm{D}_{6}$. 

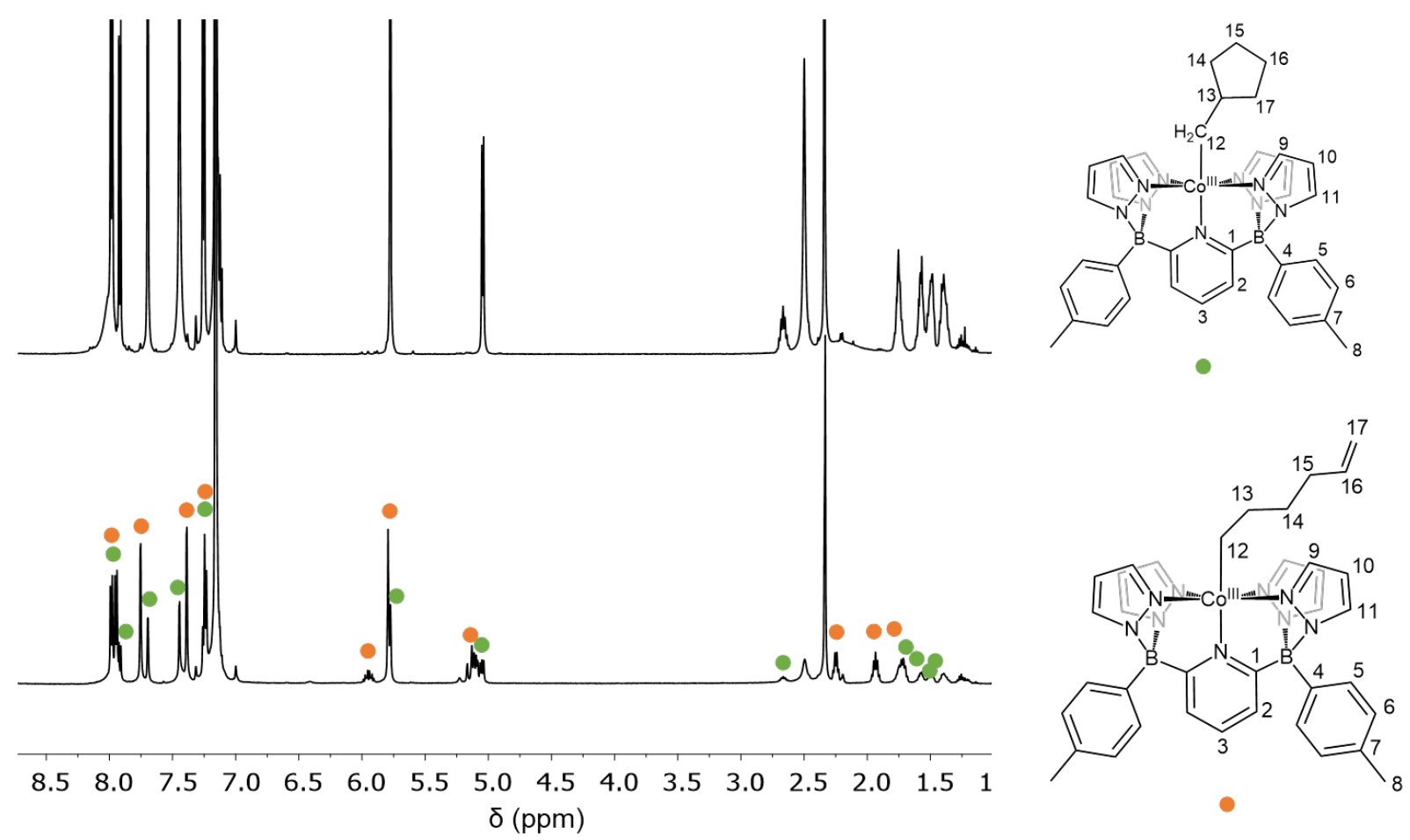

Figure S16. Stack ${ }^{1} \mathrm{H}$ NMR spectra of $2-\mathrm{CH}_{2}\left(\boldsymbol{c}-\mathrm{C}_{5} \mathrm{H}_{9}\right)$ (top) and the mixture of 2$\left(\mathrm{CH}_{2}\right)_{4} \mathrm{CH}=\mathrm{CH}_{2}$ (green dots, bottom) and 2- $\mathrm{CH}_{2}\left(\boldsymbol{c}-\mathrm{C}_{5} \mathrm{H}_{9}\right)$ (orange dots, bottom) in $\mathrm{C}_{6} \mathrm{D}_{6}$. A 2:1 ratio was determined by ${ }^{1} \mathrm{H}$ integration of the peaks corresponding to $2-\left(\mathrm{CH}_{2}\right)_{4} \mathrm{CH}=\mathrm{CH}_{2}$ vs 2$\mathrm{CH}_{2}\left(c-\mathrm{C}_{5} \mathrm{H}_{9}\right)$. 
Procedure for the reactivity of $2-\left(\mathrm{CH}_{2}\right)_{4} \mathrm{CH}=\mathrm{CH}_{2}$ in $\mathrm{C}_{6} \mathrm{D}_{6}$.

A J-Young tube equipped with a Teflon tap was charged with $5.0 \mathrm{mg}$ of $2-\left(\mathrm{CH}_{2}\right)_{4} \mathrm{CH}=\mathrm{CH}_{2}$ in $\mathrm{C}_{6} \mathrm{D}_{6}$. A ${ }^{1} \mathrm{H}$ NMR spectrum was recorded. The J-Young tube was stirred for $16 \mathrm{~h}$ and $24 \mathrm{~h}$ at room temperature and two ${ }^{1} \mathrm{H}$ NMR spectra were recorded. $A{ }^{11} \mathrm{~B}$ NMR spectrum was recorded at room temperature after $24 \mathrm{~h}$ of stirring.

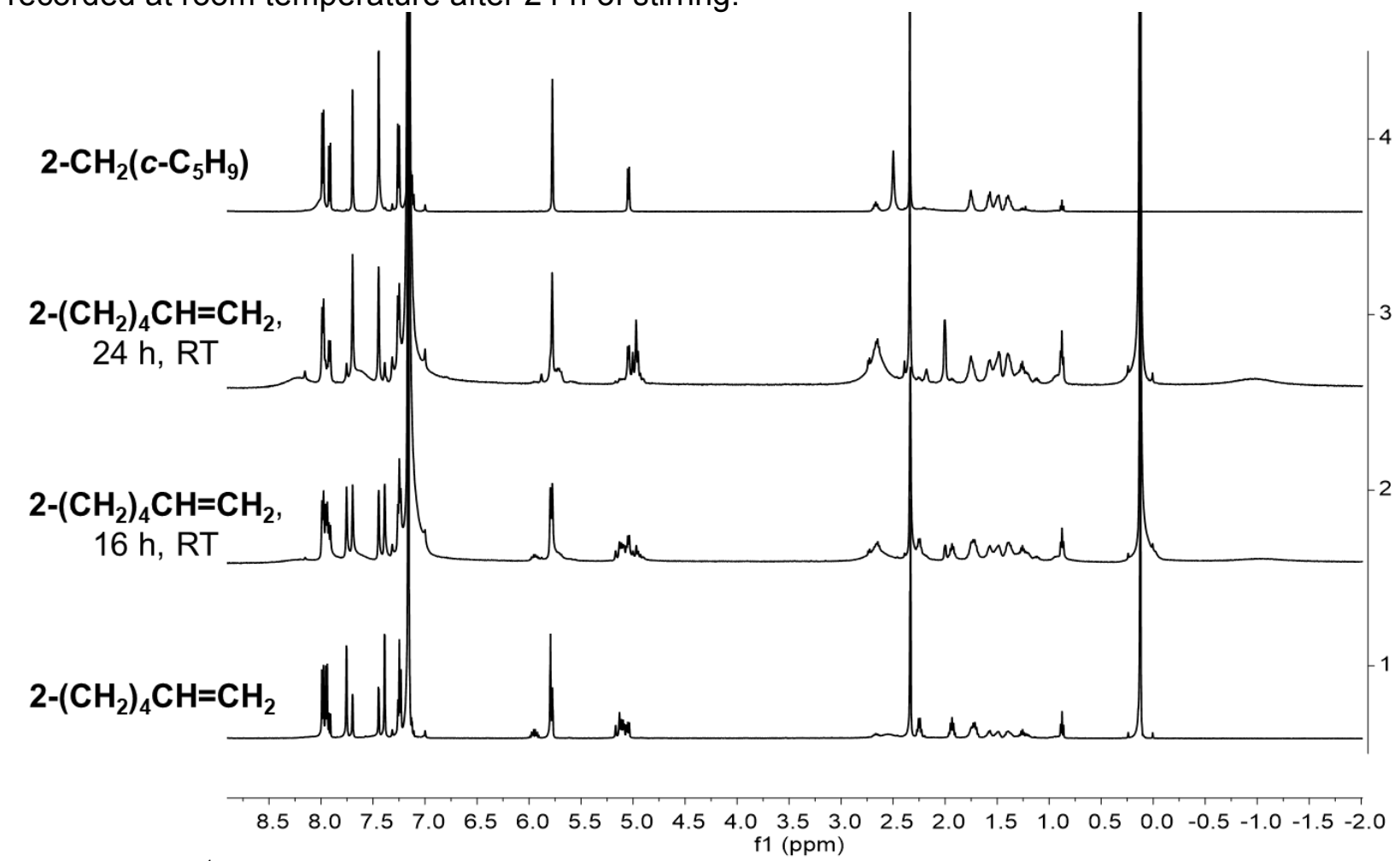

Figure S17. ${ }^{1} \mathrm{H}$ stack NMR spectra of $2-\left(\mathrm{CH}_{2}\right)_{4} \mathrm{CH}=\mathrm{CH}_{2}$ (spectrum 1), $2-\left(\mathrm{CH}_{2}\right)_{4} \mathrm{CH}=\mathrm{CH}_{2}$ at room temperature after $16 \mathrm{~h}$ in solution (spectrum 2), 2-( $\left.\mathbf{C H}_{2}\right)_{4} \mathbf{C H}=\mathbf{C H}_{2}$ at room temperature after $24 \mathrm{~h}$ in solution (spectrum 3 ) and $2-\mathrm{CH}_{2}\left(\boldsymbol{c}-\mathrm{C}_{5} \mathrm{H}_{9}\right)$ synthesized independently (spectrum 4) in $\mathrm{C}_{6} \mathrm{D}_{6}$. 


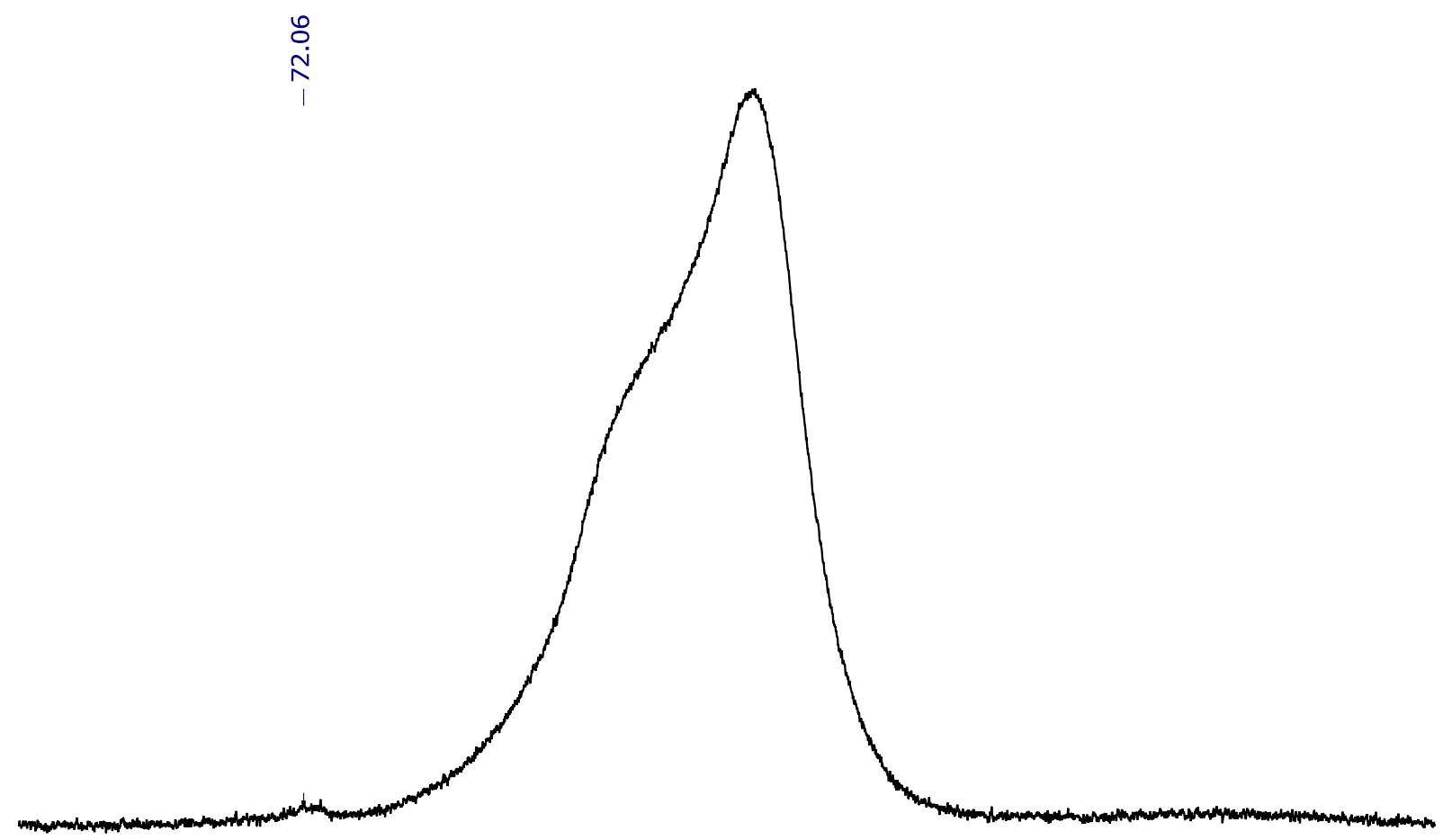

$\begin{array}{llllllllllll}110 & 90 & 70 & 50 & 30 & \begin{array}{c}10 \\ \mathrm{f} 1(\mathrm{ppm})\end{array} & -10 & -30 & -50 & -70 & -90 & -110\end{array}$

Figure S18. ${ }^{11} \mathrm{~B}$ NMR spectra of $2-\left(\mathrm{CH}_{2}\right)_{4} \mathrm{CH}=\mathrm{CH}_{2}$ at room temperature after $24 \mathrm{~h}$ in solution in $\mathrm{C}_{6} \mathrm{D}_{6}$. The broad peak corresponds to complex 3 .

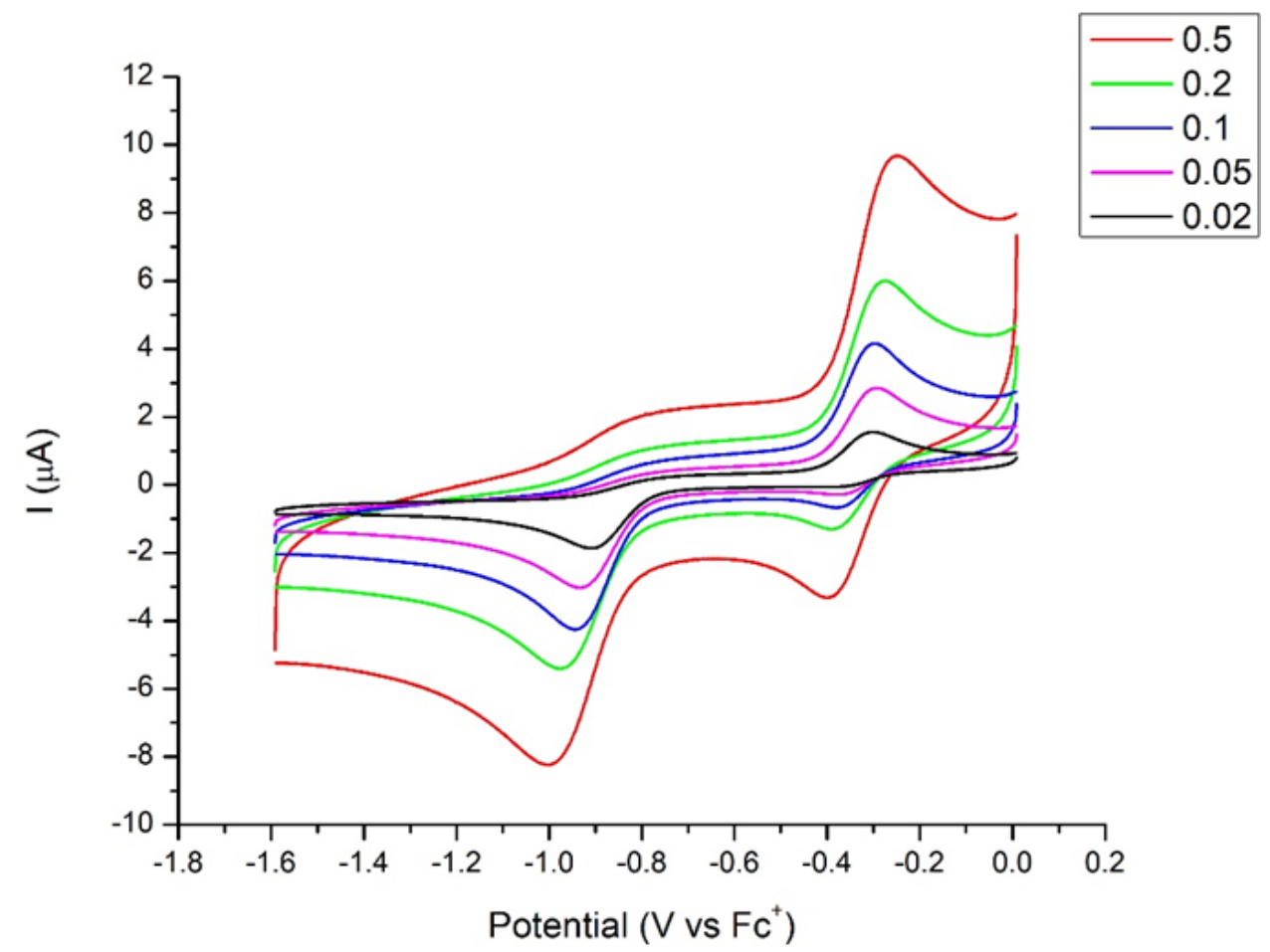

Figure S19. Refer to physical method for general information. Cyclic voltammogram of 2$\mathrm{CH}_{2} \mathrm{SiMe}_{3}$ in THF at different scan rates (from 0.02 to $0.5 \mathrm{~V} . \mathrm{s}^{-1}$ ). Concentration of 2$\mathrm{CH}_{2} \mathrm{SiMe}_{3}: 0.1 \mathrm{mM}$. 


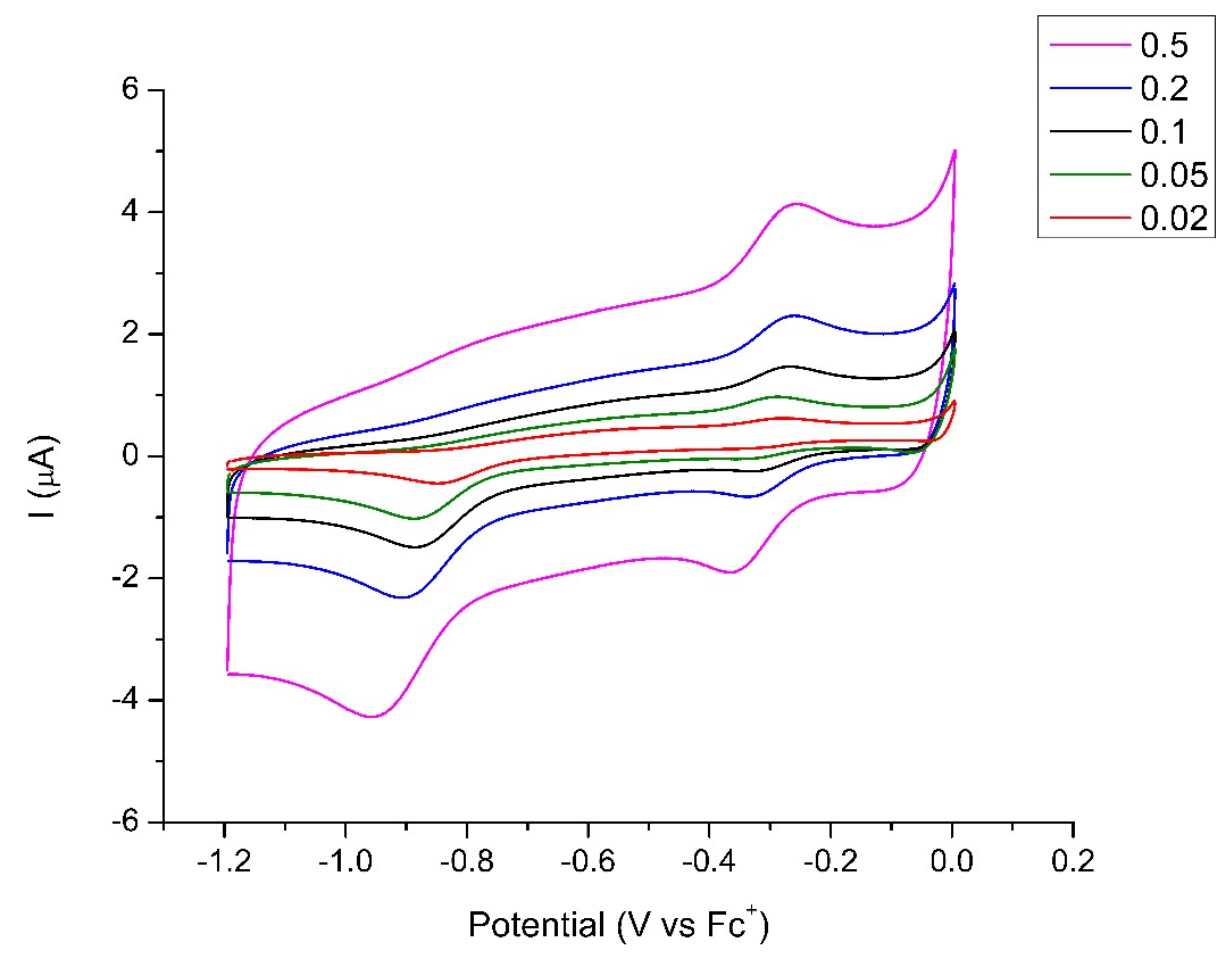

Figure S20. Refer to physical method for general information. Cyclic voltammogram of 2-

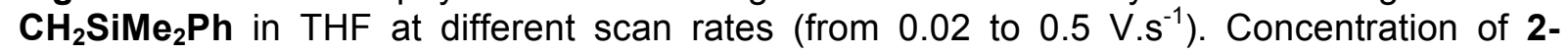
$\mathrm{CH}_{2} \mathrm{SiMe}_{2} \mathrm{Ph}$ : $0.1 \mathrm{mM}$.

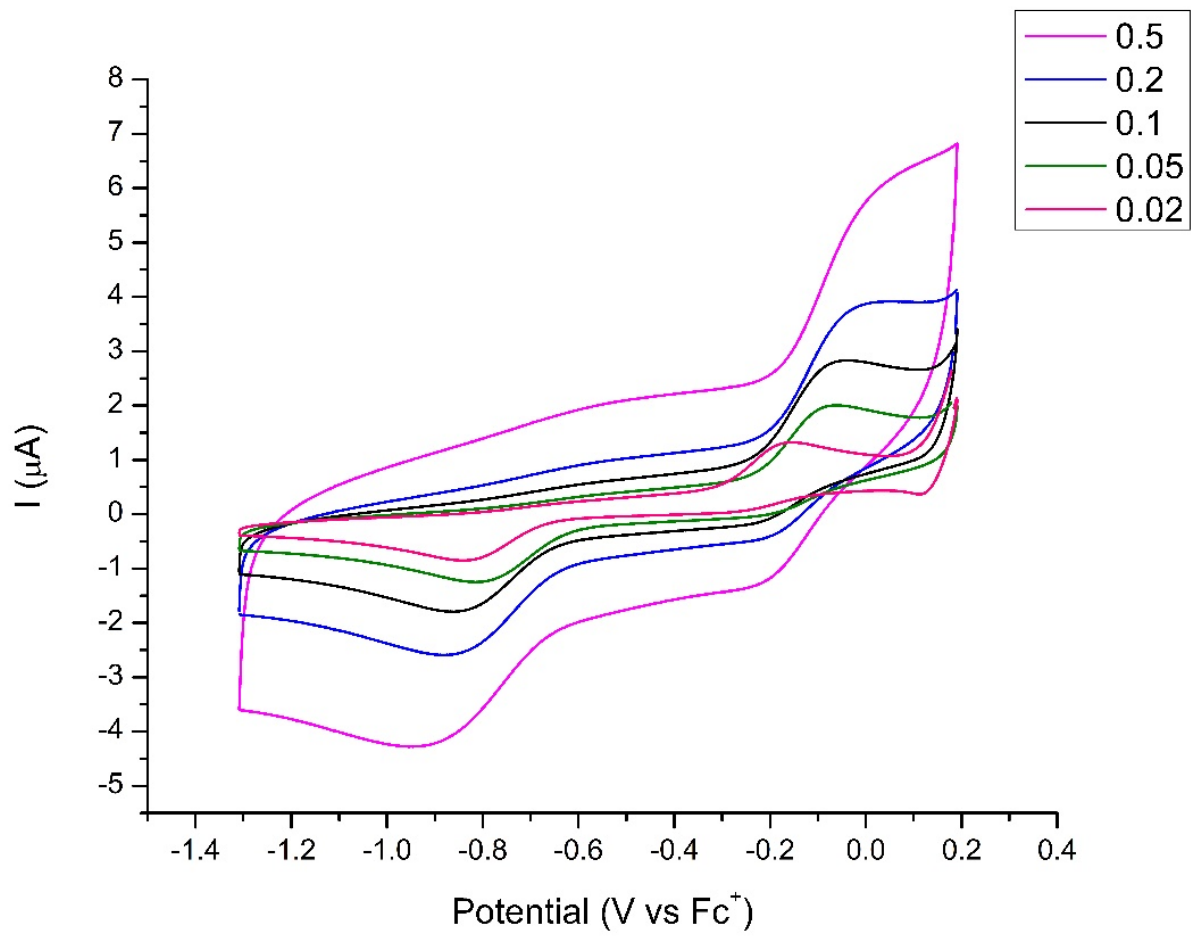

Figure S21. Refer to physical method for general information. Cyclic voltammogram of 2-'Bu in THF at different scan rates (from 0.02 to $0.5 \mathrm{~V} . \mathrm{s}^{-1}$ ). Concentration of 2-'Bu: $0.1 \mathrm{mM}$. 


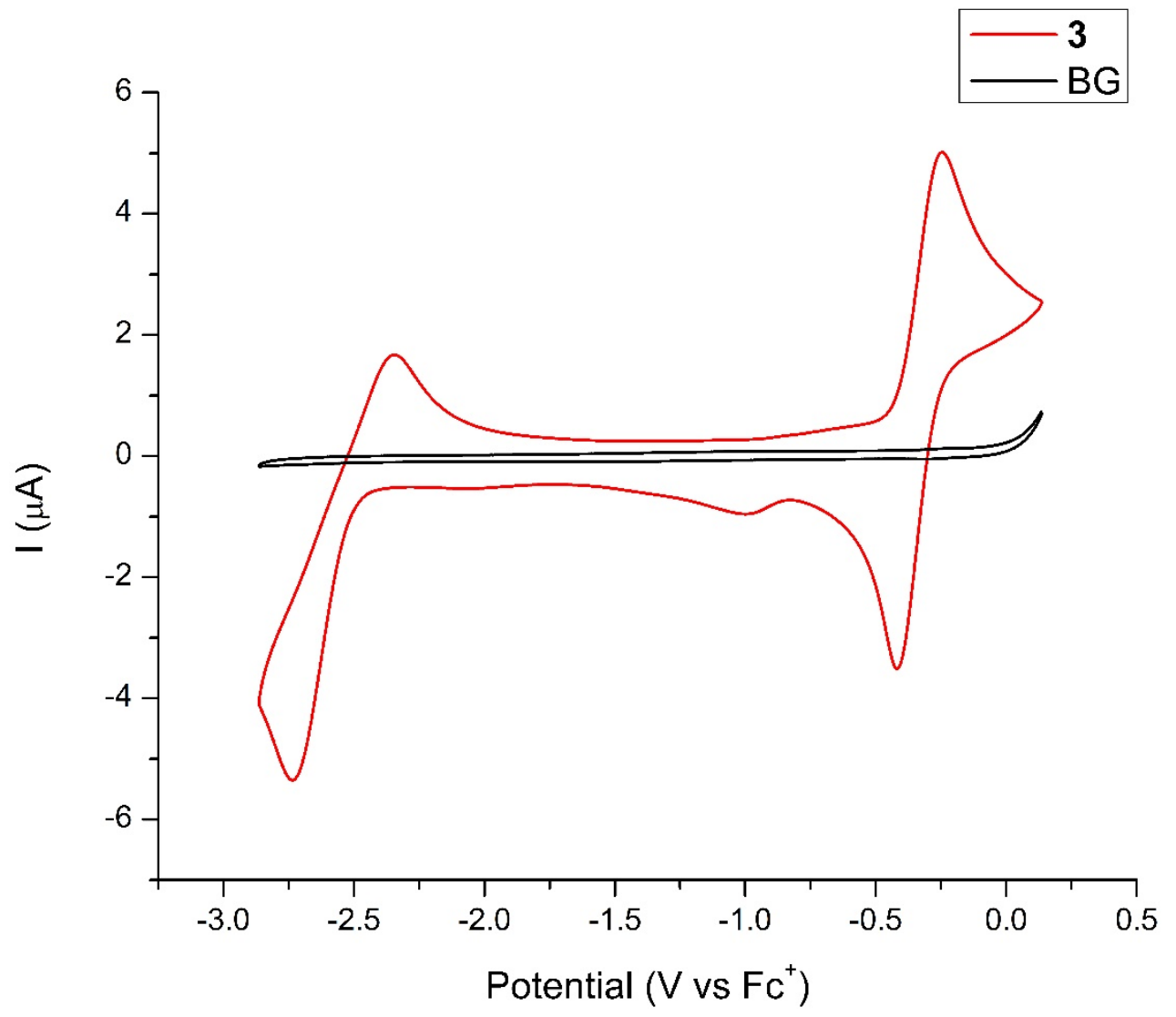

Figure S22. Refer to physical method for general information. Cyclic voltammogram of $\mathbf{3}$ in THF with a scan rate of $0.1 \mathrm{~V} . \mathrm{s}^{-1}$. The background is shown in black. Concentration of 3: 0.1 $\mathrm{mM}$. 
Procedure for the reactivity of 2-R with $\mathrm{CoCp}_{2}$ in $\mathrm{C}_{6} \mathrm{D}_{6}$.

A J-Young tube equipped with a Teflon tap was charged with $5.0 \mathrm{mg}$ of $2-\mathbf{R}$ in $\mathrm{C}_{6} \mathrm{D}_{6} . \mathrm{A}^{1} \mathrm{H}$ NMR spectrum was recorded. $\mathrm{CoCp}_{2}(1.1 \mathrm{eq})$ was added at room temperature and another ${ }^{1} \mathrm{H}$ NMR spectrum was recorded 15 mins after the addition of $\mathrm{CoCp}_{2}$. A ${ }^{11} \mathrm{~B}$ NMR spectrum was recorded to detect complex 3.

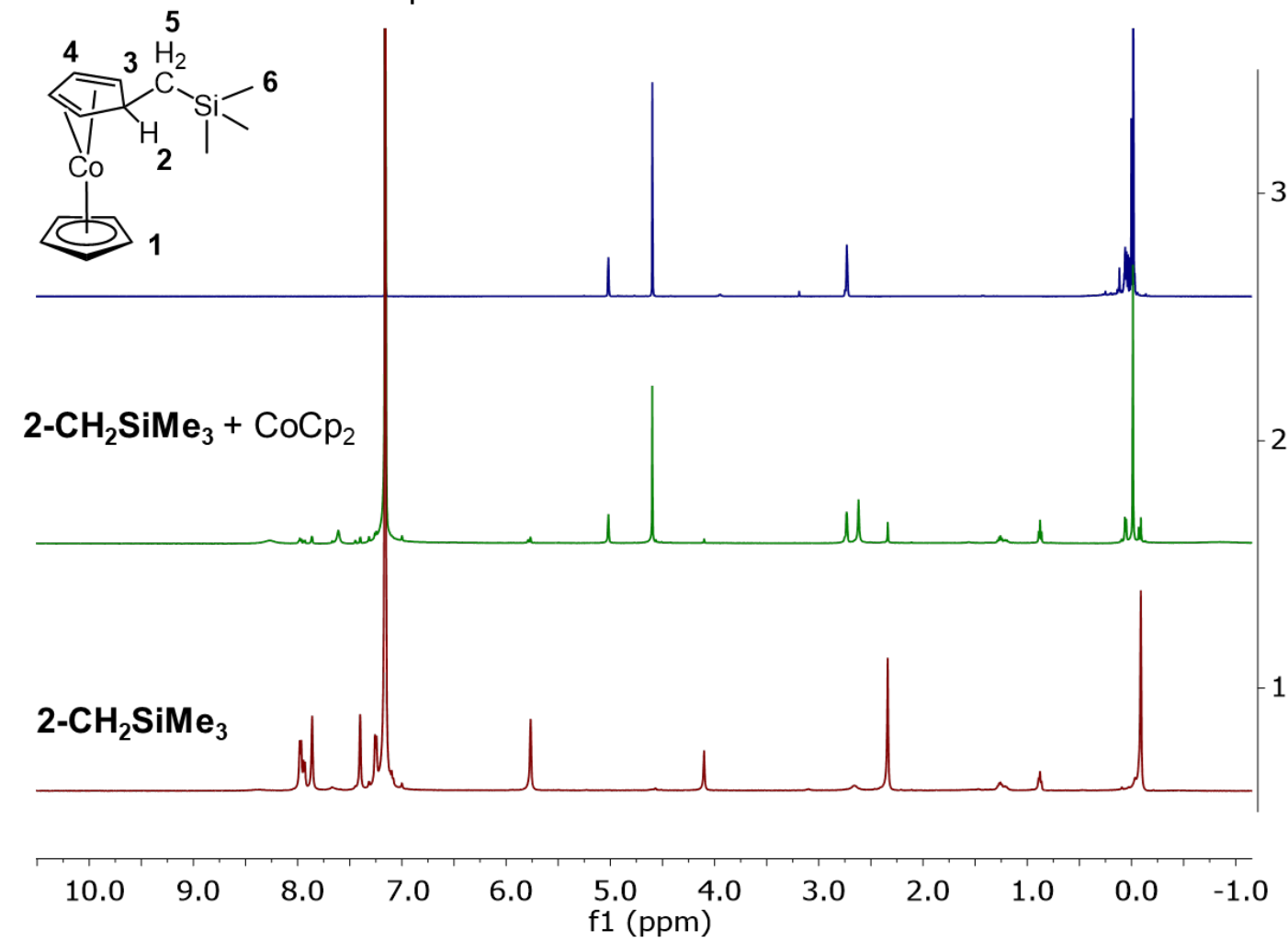

Figure S23. ${ }^{1} \mathrm{H}$ stack NMR spectra of $2-\mathrm{CH}_{2} \mathrm{SiMe}_{3}$ (spectrum 1), $2-\mathrm{CH}_{2} \mathrm{SiMe}_{3}+\mathrm{CoCp}_{2}$ (spectrum 2) and $\mathrm{I}-\mathrm{CH}_{2} \mathrm{SiMe}_{3}$ (spectrum 3) in $\mathrm{C}_{6} \mathrm{D}_{6}$.

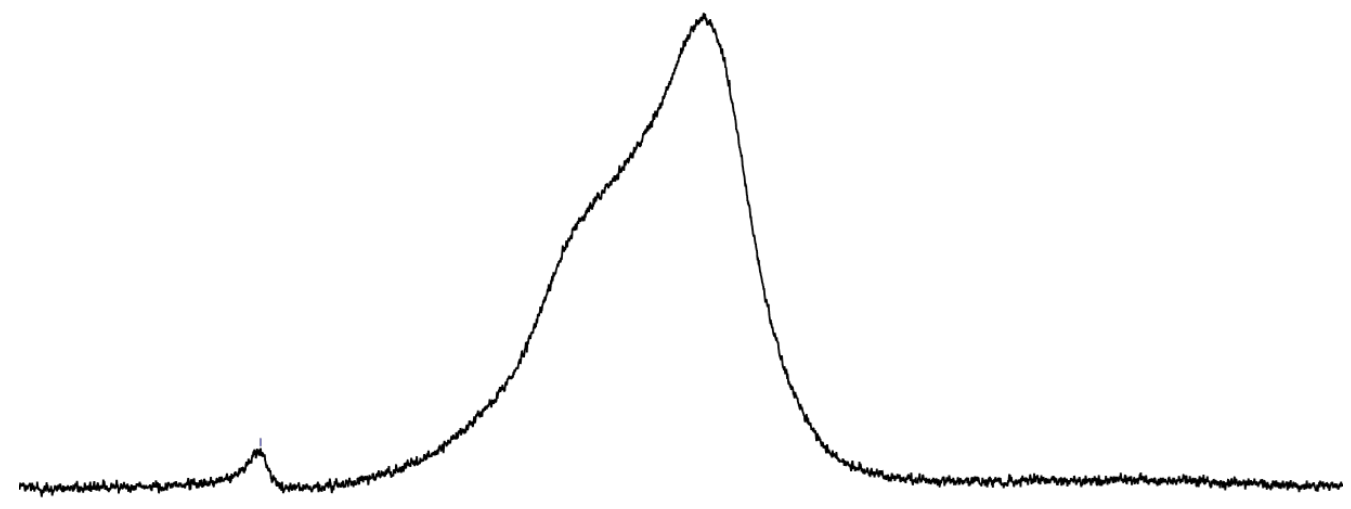

$\begin{array}{llllllllllll}110 & 90 & 70 & 50 & 30 & 10 & -10 & -30 & -50 & -70 & -90 & -110\end{array}$

Figure S24. ${ }^{11} \mathrm{~B}$ NMR spectrum of $2-\mathrm{CH}_{2} \mathrm{SiMe}_{3}+\mathrm{CoCp}_{2}$ in $\mathrm{C}_{6} \mathrm{D}_{6}$. 


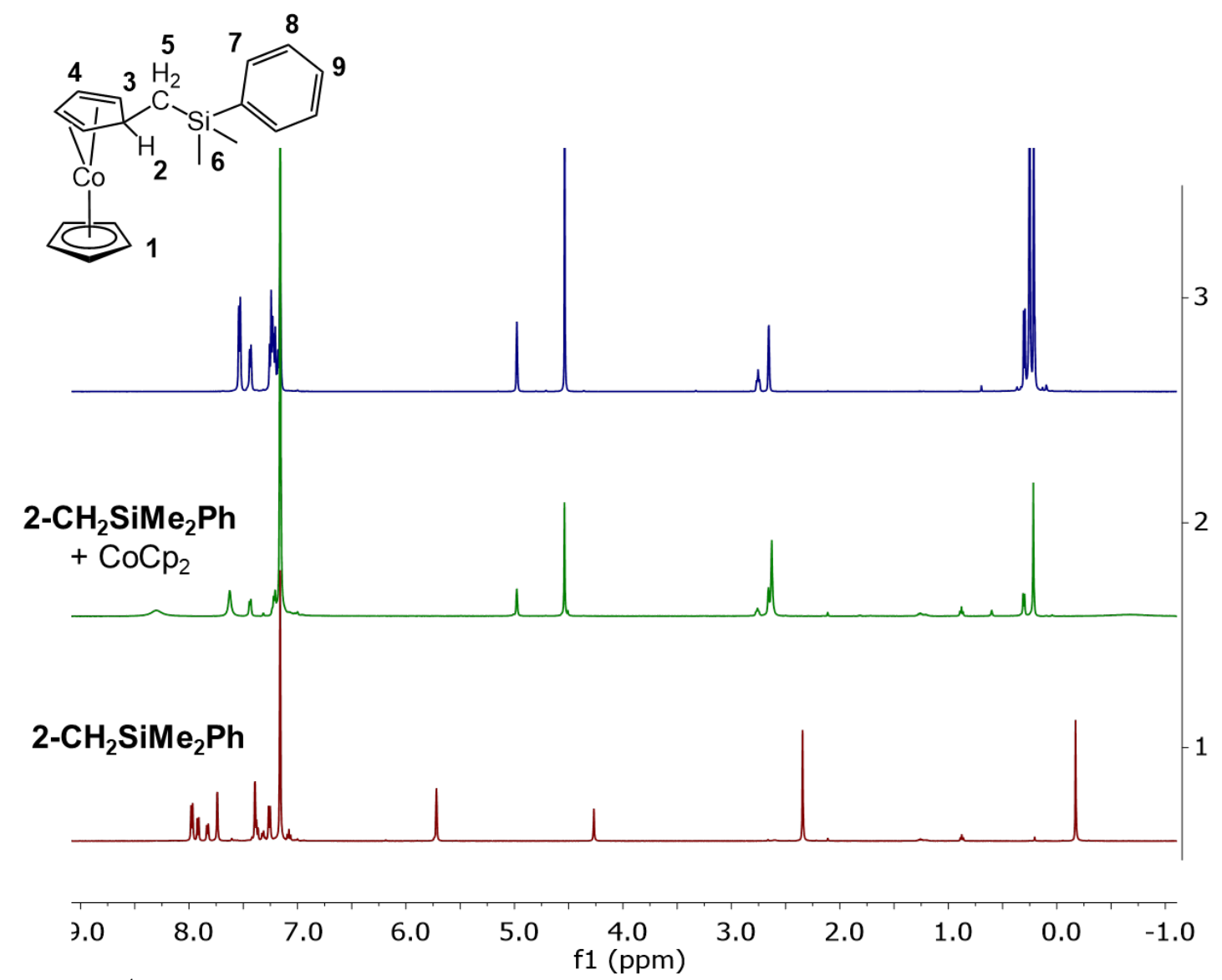

Figure S25. ${ }^{1} \mathrm{H}$ stack NMR spectra of $2-\mathrm{CH}_{2} \mathrm{SiMe}{ }_{2} \mathrm{Ph}$ (spectrum 1), $2-\mathrm{CH}_{2} \mathrm{SiMe}{ }_{2} \mathrm{Ph}+\mathrm{CoCp}$ (spectrum 2) and I- $\mathrm{CH}_{2} \mathrm{SiMe}_{2} \mathrm{Ph}$ (spectrum 3 ) in $\mathrm{C}_{6} \mathrm{D}_{6}$.

Ñ

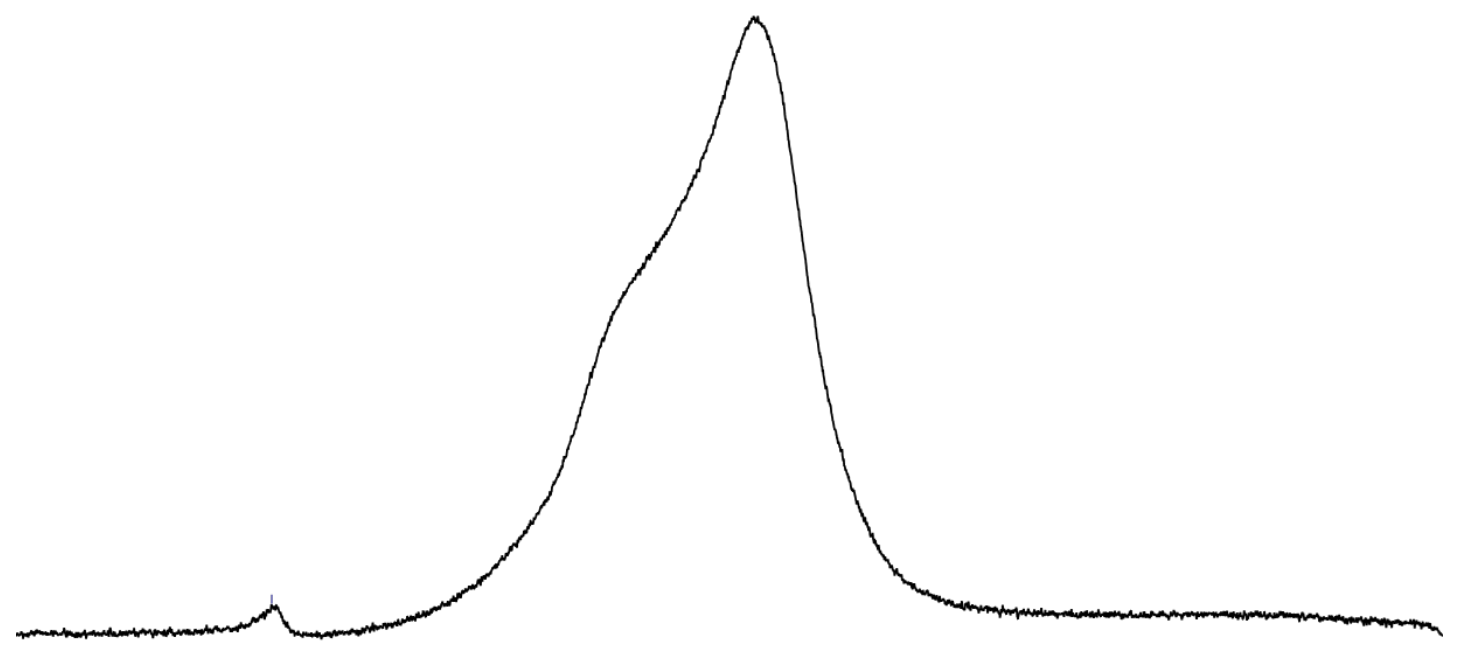

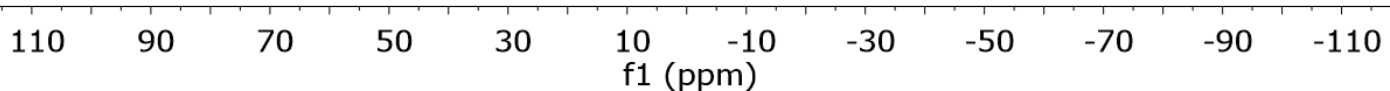

Figure S26. ${ }^{11} \mathrm{~B}$ NMR spectrum of $2-\mathrm{CH}_{2} \mathrm{SiMe}_{2} \mathrm{Ph}+\mathrm{CoCp}_{2}$ in $\mathrm{C}_{6} \mathrm{D}_{6}$. 


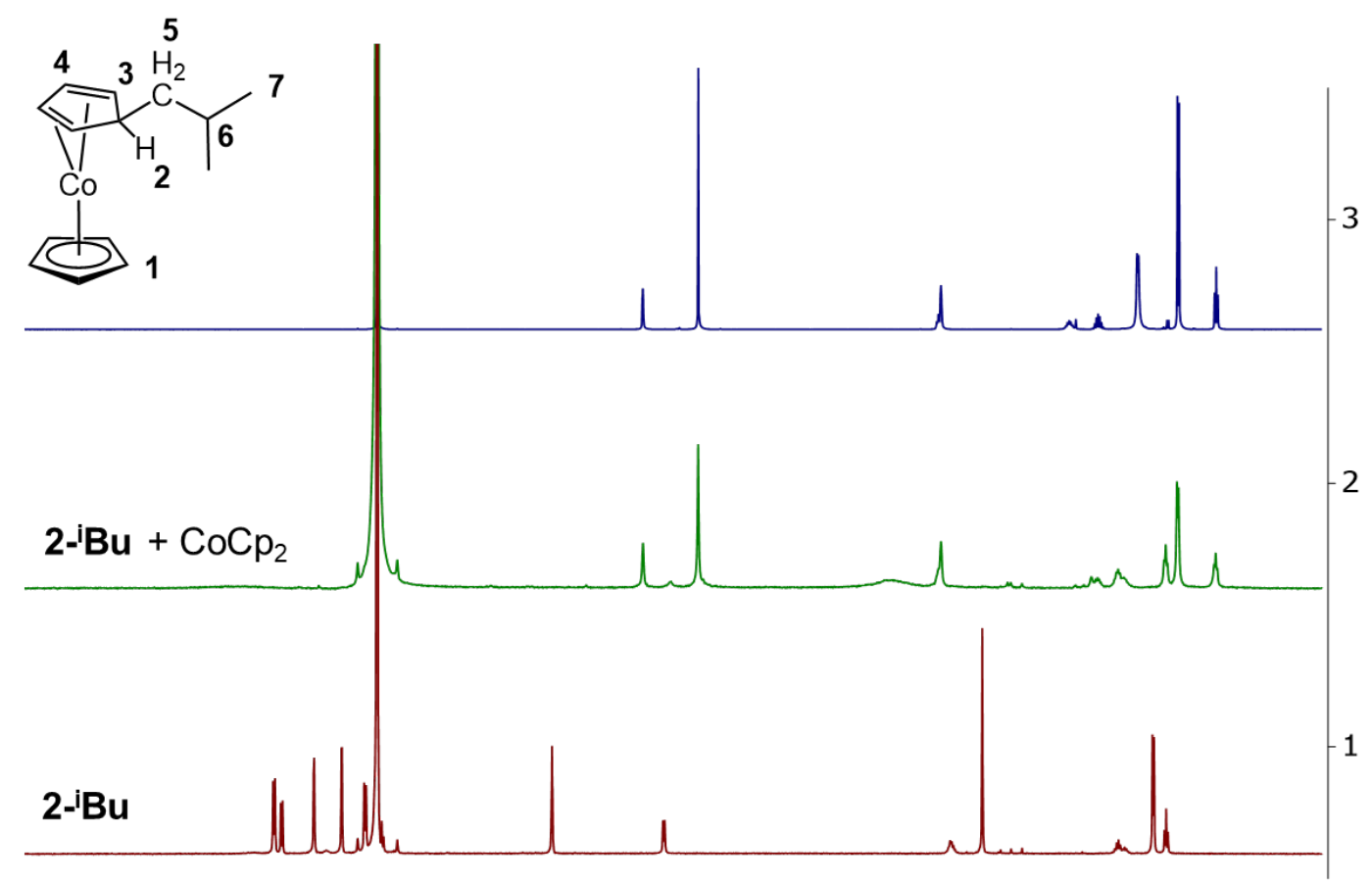

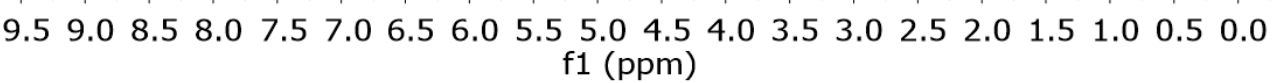

Figure S27. ${ }^{1} \mathrm{H}$ stack NMR spectra of $2-{ }^{\mathrm{i}} \mathbf{B u}$ (spectrum 1), 2-'Bu + $\mathrm{CoCp}$ (spectrum 2) and I$\mathrm{CH}_{2} \mathrm{CH}\left(\mathrm{CH}_{3}\right)_{2}$ (spectrum 3) in $\mathrm{C}_{6} \mathrm{D}_{6}$.

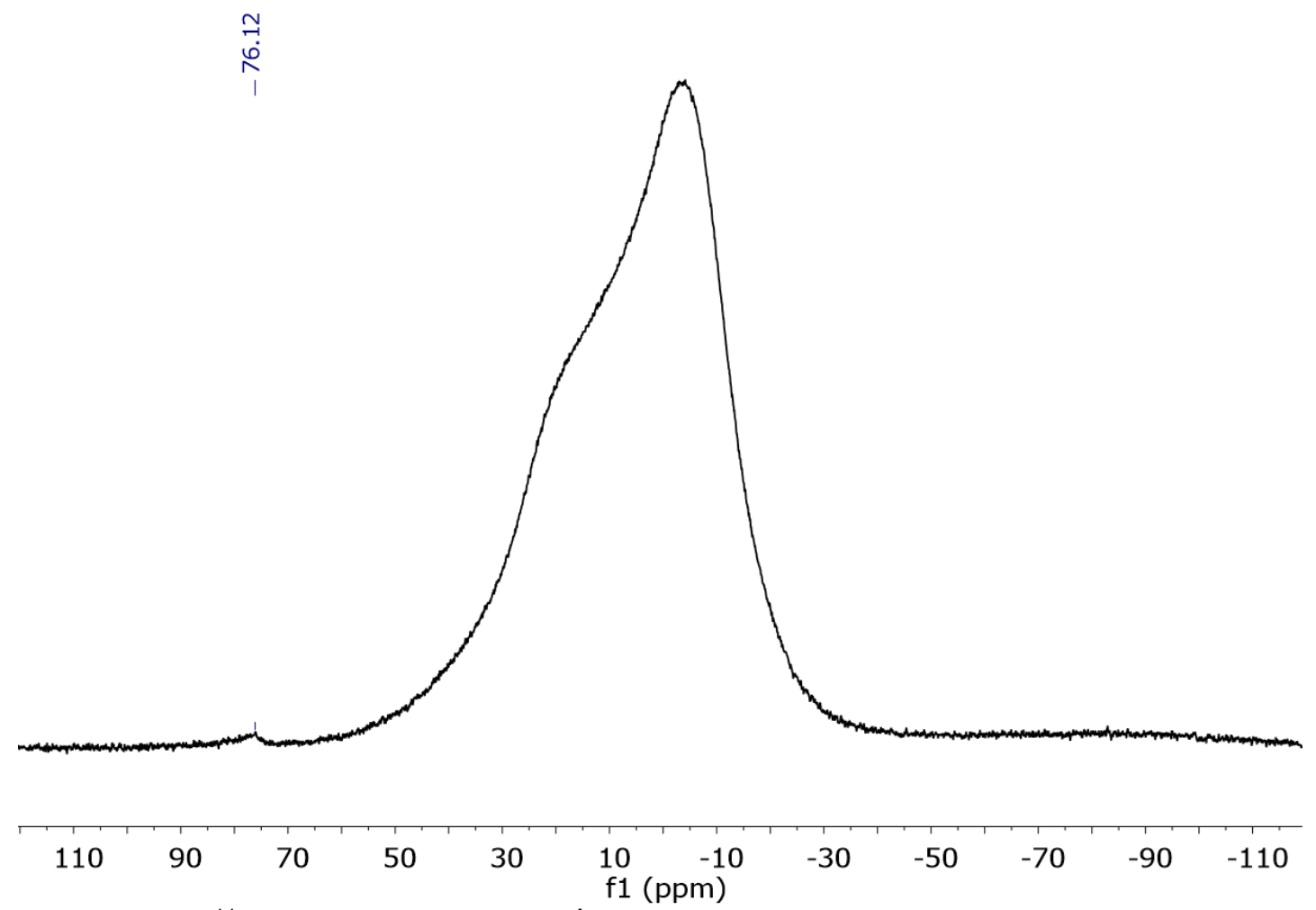

Figure S28. ${ }^{11} \mathrm{~B}$ NMR spectrum of 2-'Bu + $\mathrm{CoCp}_{2}$ in $\mathrm{C}_{6} \mathrm{D}_{6}$. 


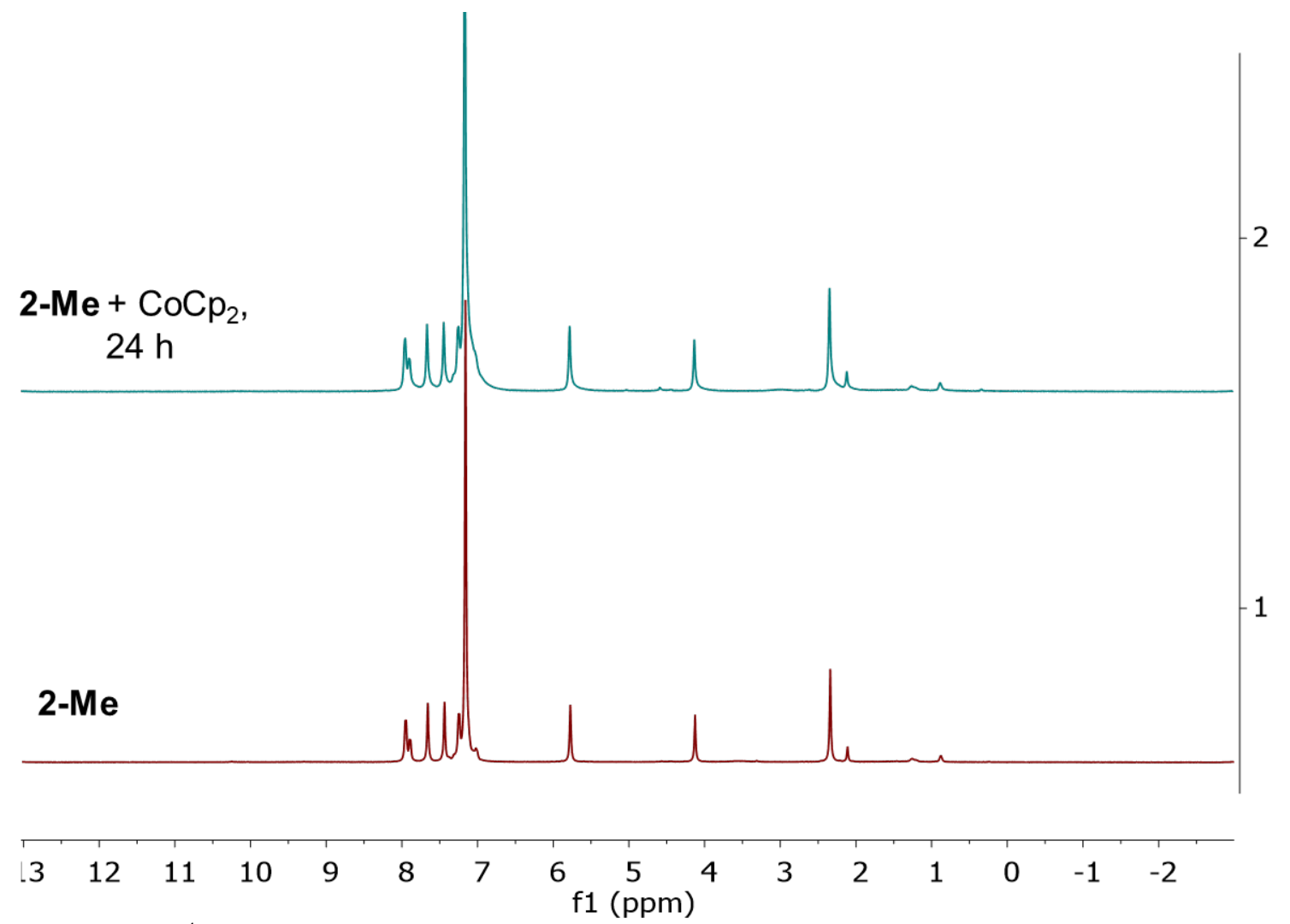

Figure S29. ${ }^{1} \mathrm{H}$ stack NMR spectra of 2-Me (spectrum 1) and $2-\mathbf{M e}+\mathrm{CoCp}_{2}\left(\mathrm{spectrum}_{2}\right)$ in $\mathrm{C}_{6} \mathrm{D}_{6}$. 


$$
{ }^{*} \mathrm{C}_{2} \mathrm{H}_{6} \quad{ }^{*} \mathrm{CH}_{4}
$$
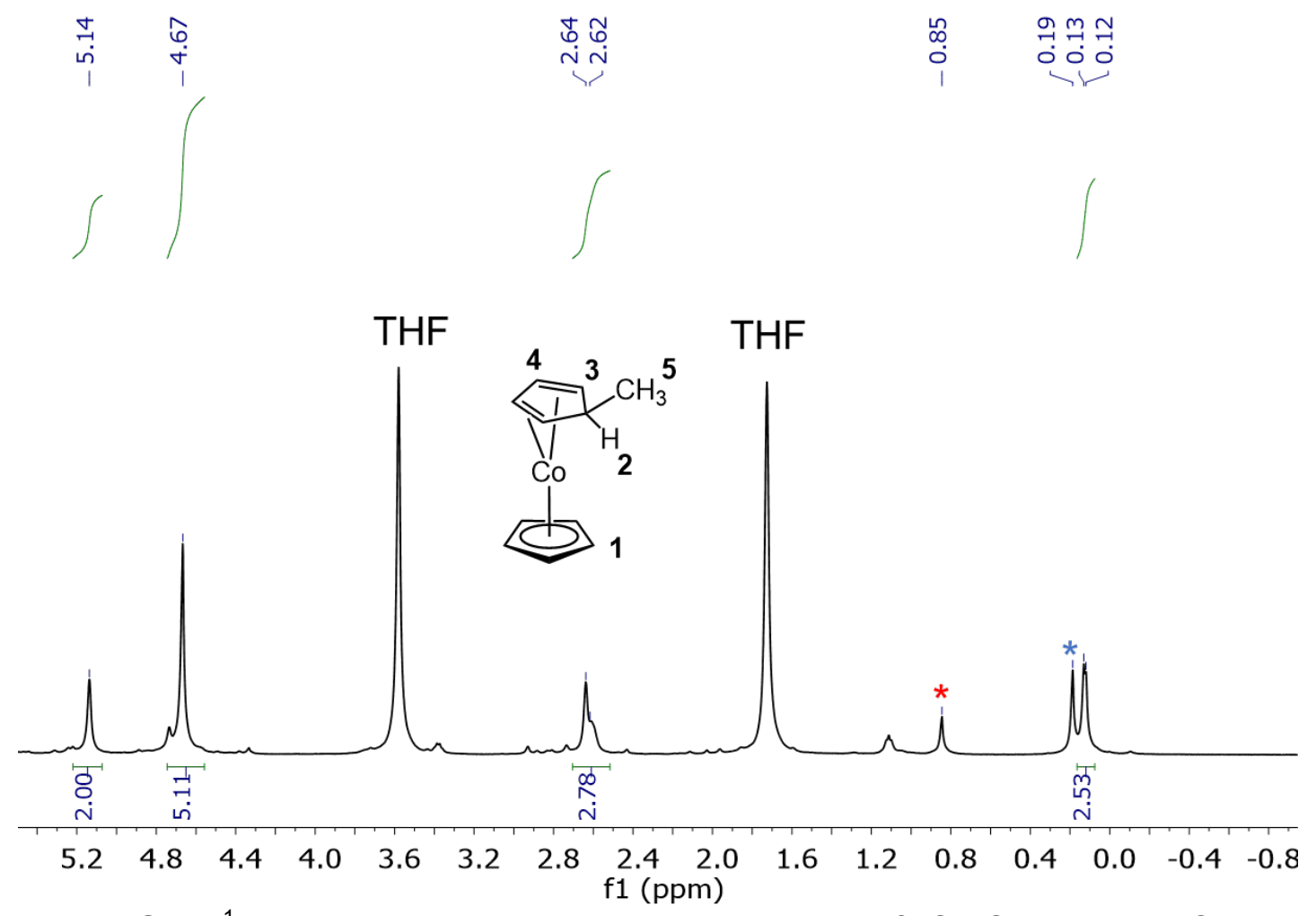

Figure S30. ${ }^{1} \mathrm{H}$ NMR spectrum (diamagnetic window) of $\left[\mathrm{Cp}_{2} \mathrm{Co}\right]\left[\mathrm{PF}_{6}\right]+\mathrm{LiCH}_{3}$ in THF-d8. The $\mathrm{CH}_{4}$ peak is overlapping with the $-\mathrm{CH}_{3}$ protons $(\mathrm{H} 5)$.

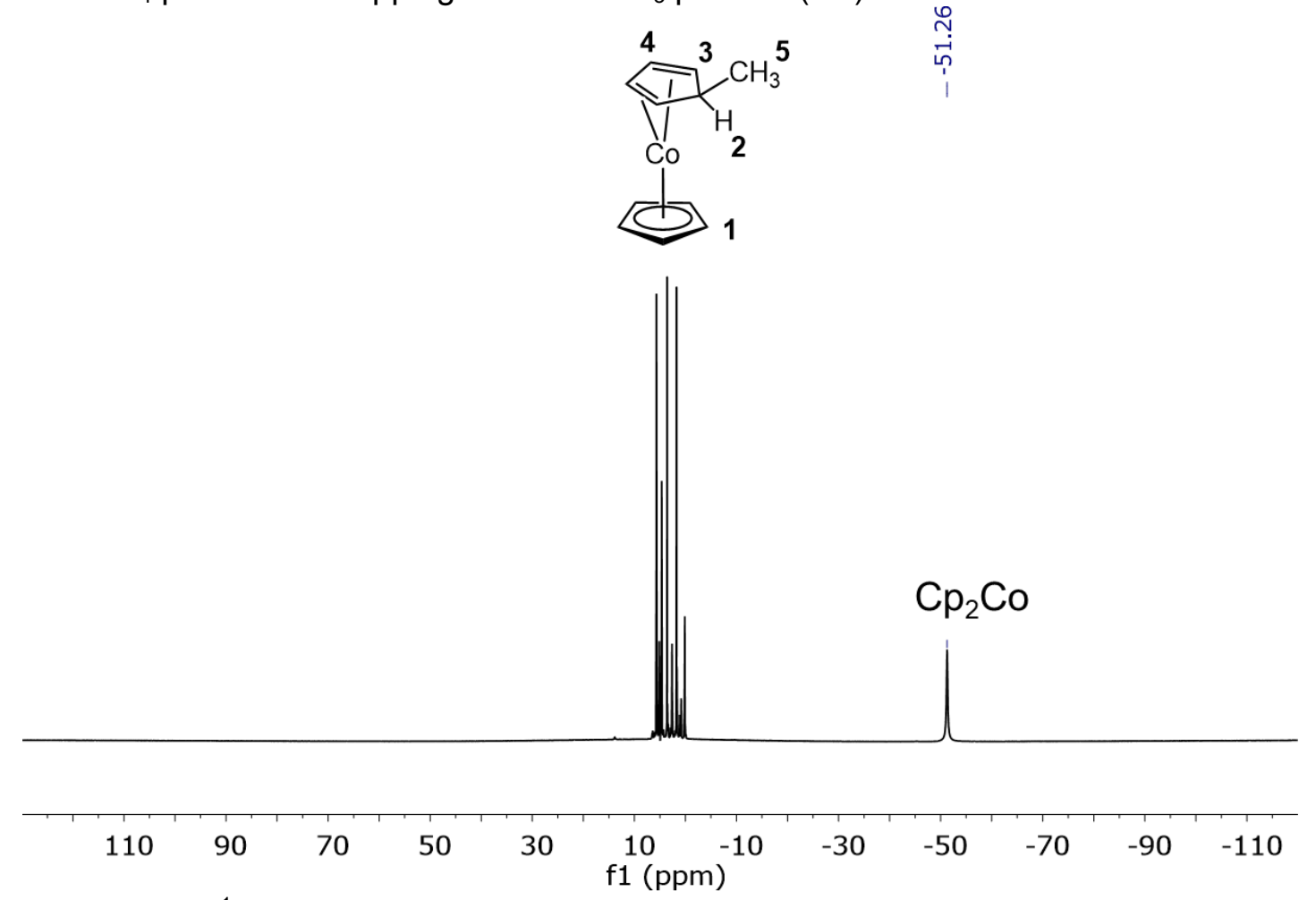

Figure S31. ${ }^{1} \mathrm{H}$ NMR spectrum (large window) of $\left[\mathrm{Cp}_{2} \mathrm{Co}\right]\left[\mathrm{PF}_{6}\right]+\mathrm{LiCH}_{3}$ in THF-d8. Cp $\mathrm{C}_{2} \mathrm{Co}$ is detected in this reaction at $-51.3 \mathrm{ppm}$. 


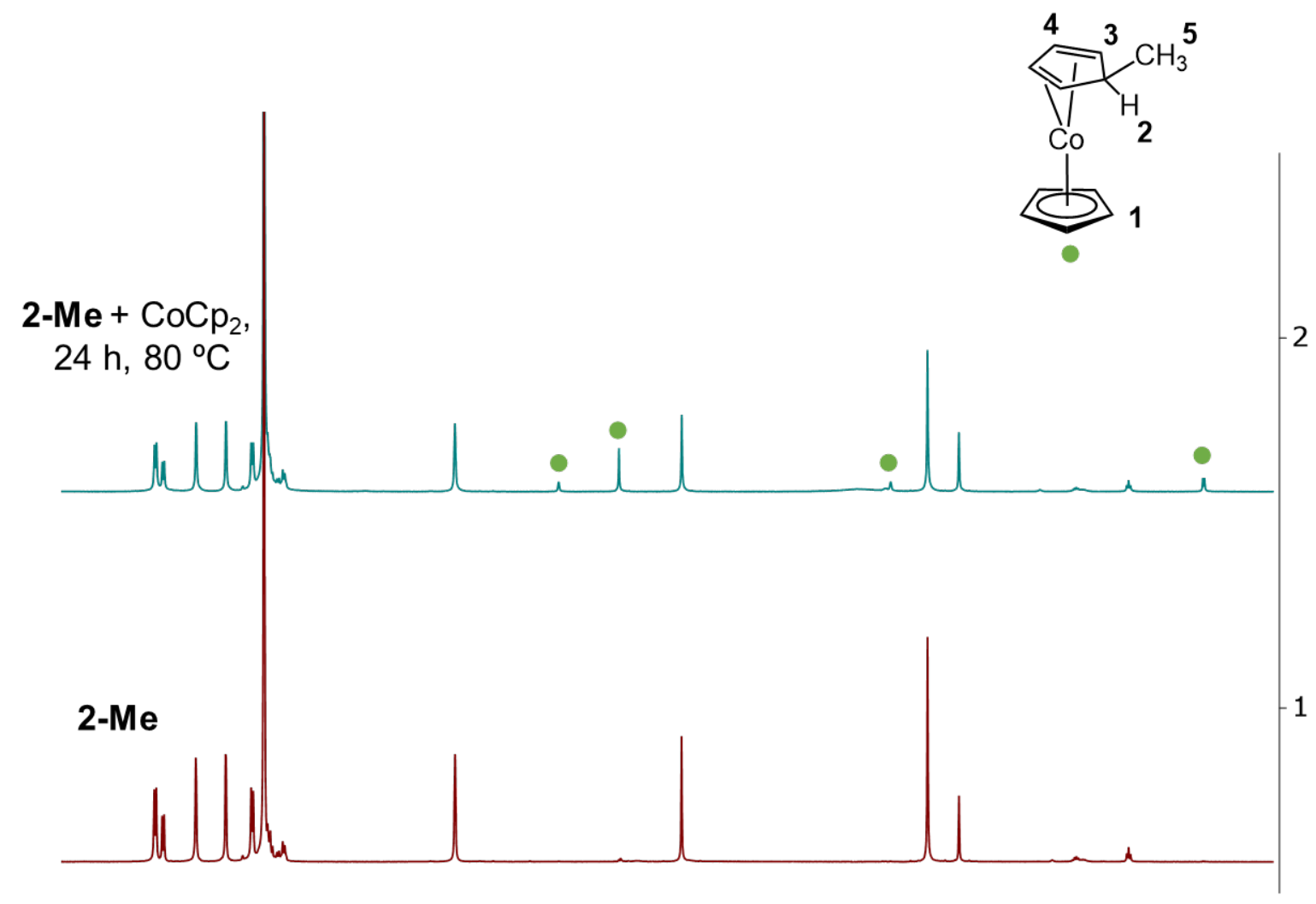

$\begin{array}{llllllllllllllllll}8.5 & 8.0 & 7.5 & 7.0 & 6.5 & 6.0 & 5.5 & 5.0 & 4.5 & 4.0 & 3.5 & 3.0 & 2.5 & 2.0 & 1.5 & 1.0 & 0.5 & 0.0\end{array}$ f1 (ppm)

Figure S32. ${ }^{1} \mathrm{H}$ stack NMR spectra of 2-Me (spectrum 1) and 2-Me $+\mathrm{CoCp}_{2}$ at $80{ }^{\circ} \mathrm{C}$ (spectrum 2) in $\mathrm{C}_{6} \mathrm{D}_{6}$. 
Procedure for the reactivity of 2-R with $\mathrm{HNTf}_{2}$ in $\mathrm{C}_{6} \mathrm{D}_{6}$.

A J-Young tube equipped with a Teflon tap was charged with $5.0 \mathrm{mg}$ of $\mathbf{2}-\mathbf{R}$ in $\mathrm{C}_{6} \mathrm{D}_{6} . \mathrm{A}^{1} \mathrm{H}$ NMR spectrum was recorded. A stock solution of $\mathrm{HNTf}_{2}$ in $\mathrm{C}_{6} \mathrm{D}_{6}$ was prepared and 1 eq of $\mathrm{HNTf}_{2}$ was added to the J-Young tube. A ${ }^{1} \mathrm{H}$ NMR spectrum was recorded at room temperature.

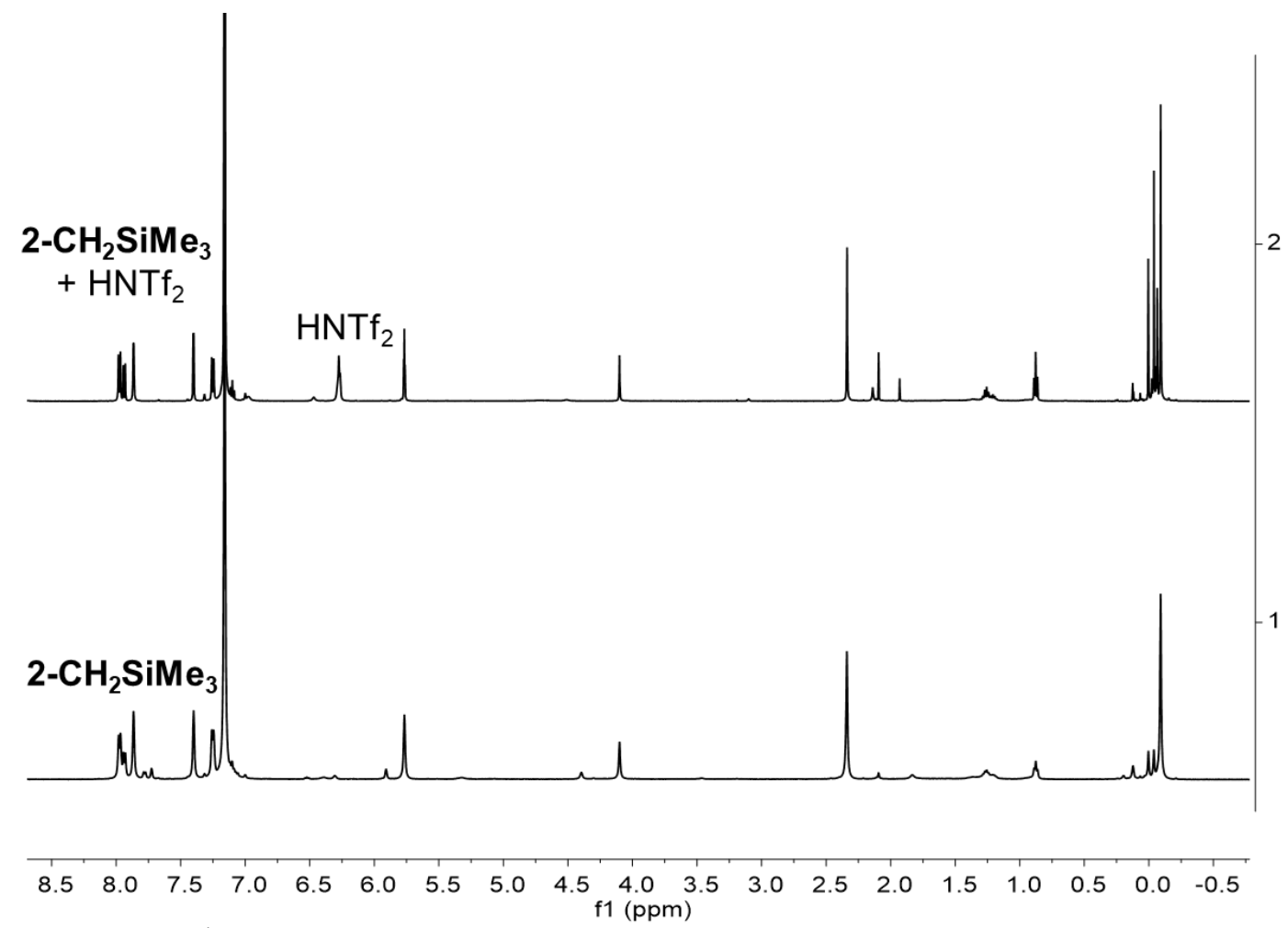

Figure S33. ${ }^{1} \mathrm{H}$ stack NMR spectra of $2-\mathrm{CH}_{2} \mathrm{SiMe}_{3}$ (spectrum 1) and $2-\mathrm{CH}_{2} \mathrm{SiMe}_{3}+\mathrm{HNTf}_{2}$ at room temperature (spectrum 2) in $\mathrm{C}_{6} \mathrm{D}_{6}$.

Figure S34. Picture of the purple precipitate formed by the reaction of 2-Me with $\mathrm{HNTf}_{2}$ in a J-Young tube. 


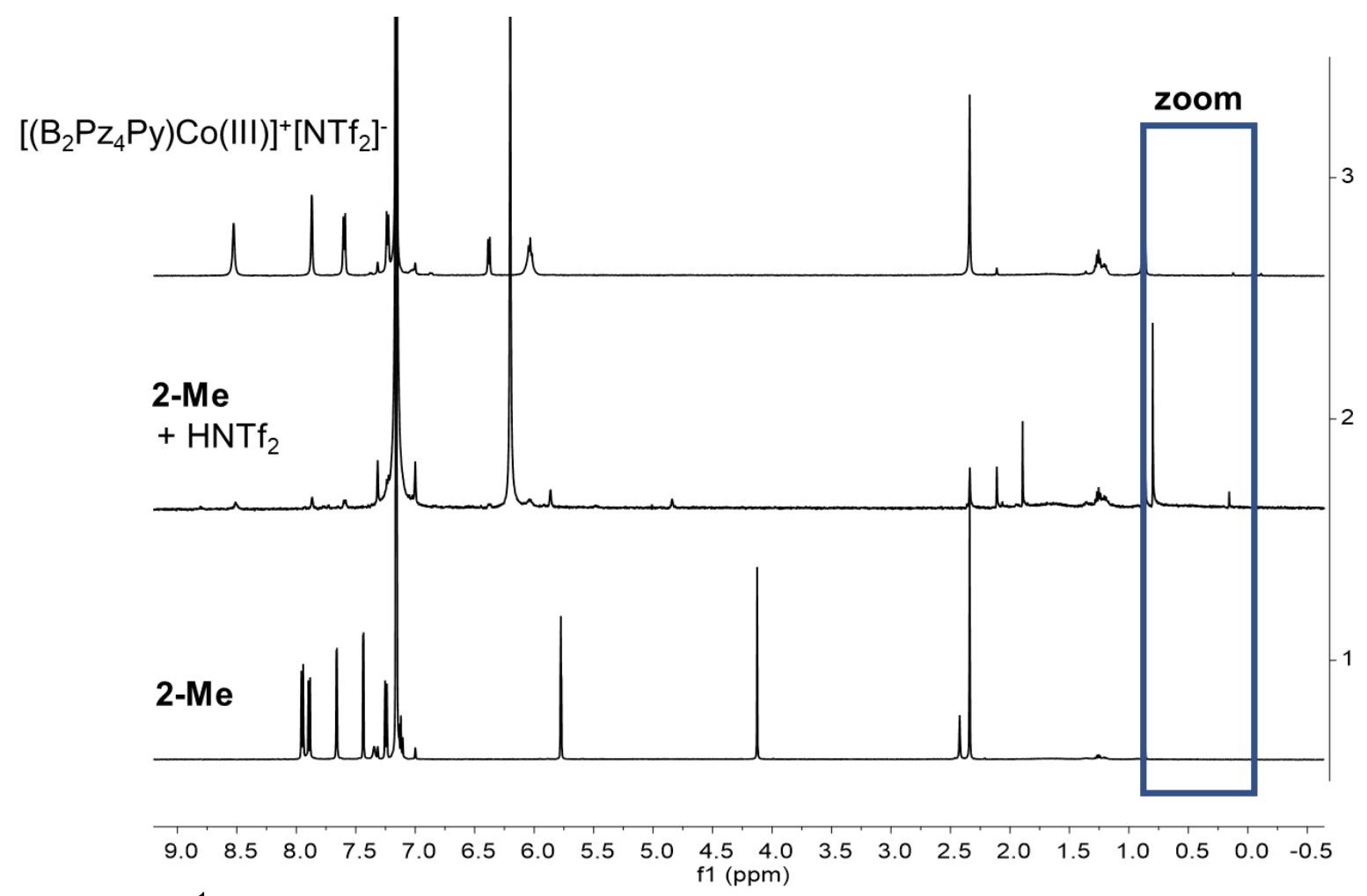

Figure S35. ${ }^{1} \mathrm{H}$ stack NMR spectra of 2-Me (spectrum 1), 2-Me $+\mathrm{HNTf}_{2}$ (spectrum 2) and $\left[\left(\mathrm{B}_{2} \mathrm{Pz}_{4} \mathrm{Py}\right) \mathrm{Co}(\mathrm{III})\right]^{+}\left[\mathrm{NTf}_{2}\right]^{-}$(spectrum 3$)$ in $\mathrm{C}_{6} \mathrm{D}_{6}$. The zoomed section is shown in Figure $\mathrm{S} 34$.

$\left[\left(\mathrm{B}_{2} \mathrm{Pz}_{4} \mathrm{Py}\right) \mathrm{Co}(\mathrm{III})\right]^{+}\left[\mathrm{NTf}_{2}\right]^{-}$

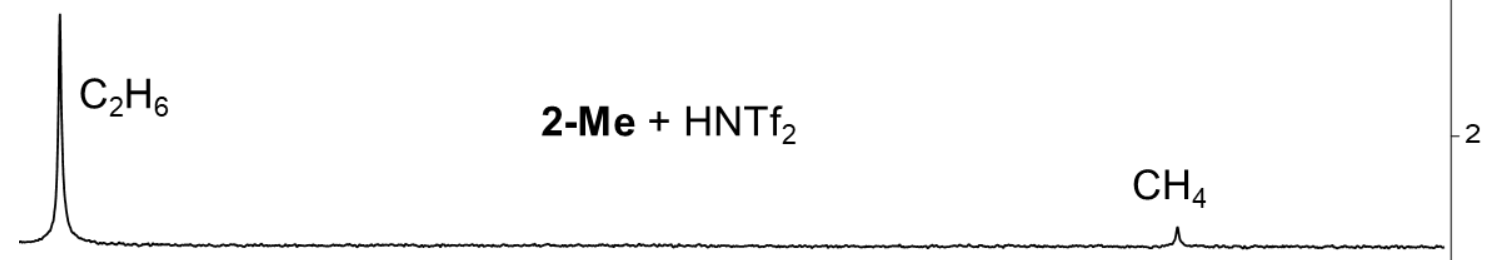

\section{2-Me}

$\begin{array}{llllllllllllllll}0.80 & 0.75 & 0.70 & 0.65 & 0.60 & 0.55 & 0.50 & 0.45 & 0.40 & 0.35 & 0.30 & 0.25 & 0.20 & 0.15 & 0.10 & 0.05\end{array}$

Figure S36. Zoomed section from ${ }^{1} \mathrm{H}$ stack NMR spectra of 2-Me (spectrum 1), 2-Me + $\mathrm{HNTf}_{2}$ (spectrum 2) and $\left[\left(\mathrm{B}_{2} \mathrm{Pz}_{4} \mathrm{Py}\right) \mathrm{Co}(\mathrm{III})\right]^{+}\left[\mathrm{NTf}_{2}\right]^{-}$(spectrum 3) in $\mathrm{C}_{6} \mathrm{D}_{6}$. The zoomed section shows the formation of ethane and methane. 


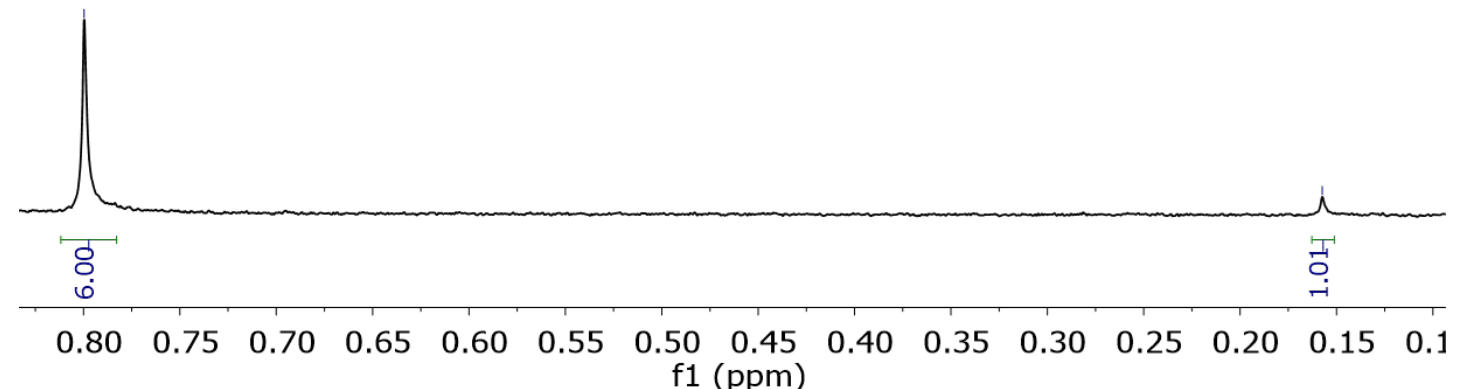

Figure S37. Zoomed section from ${ }^{1} \mathrm{H}$ NMR spectrum of 2-Me $+\mathrm{HNTf}_{2}$ with integrations.
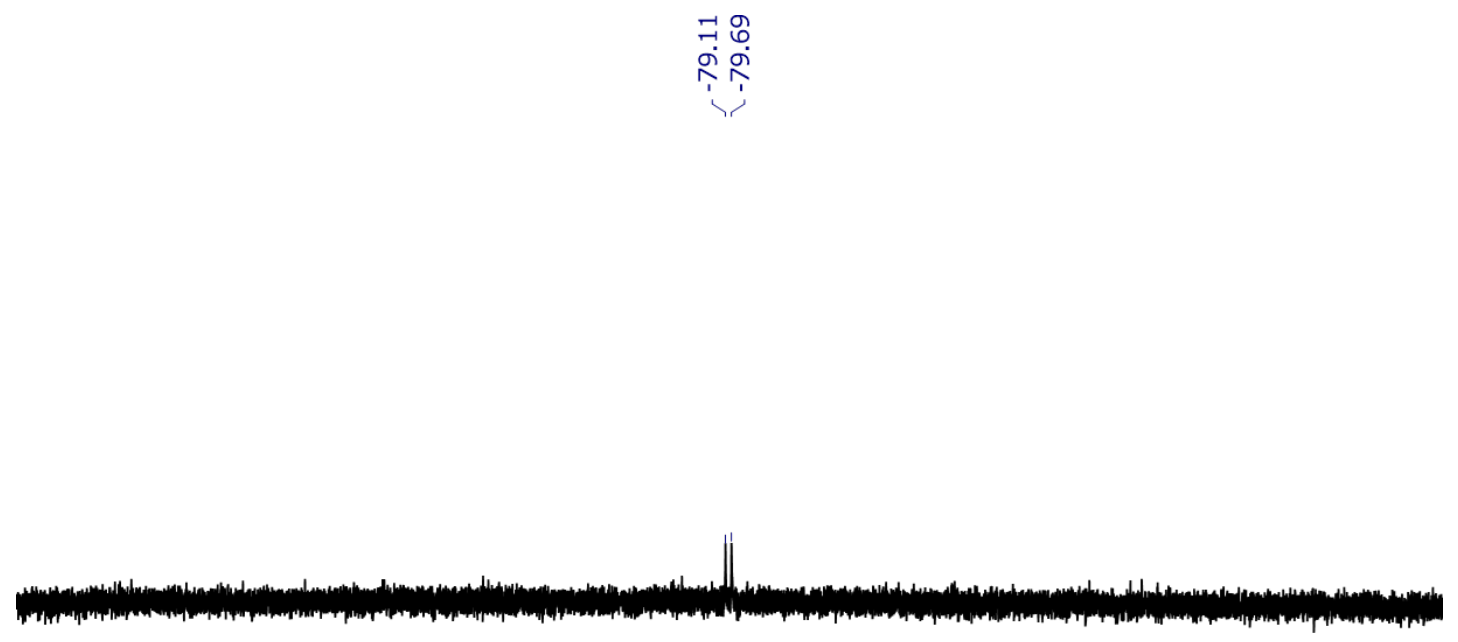

\begin{tabular}{|c|c|c|c|c|c|c|c|c|c|c|c|c|c|}
\hline-15 & -25 & -35 & -45 & -55 & -65 & $\begin{array}{l}-75 \\
\mathrm{f} 1(\mathrm{p}\end{array}$ & $m)^{-85}$ & -95 & -105 & -115 & -125 & -135 & $-14 E$ \\
\hline
\end{tabular}

Figure S38. ${ }^{19} \mathrm{~F}$ NMR spectrum of 2-Me $+\mathrm{HNTf}_{2}$ in $\mathrm{C}_{6} \mathrm{D}_{6}$. 


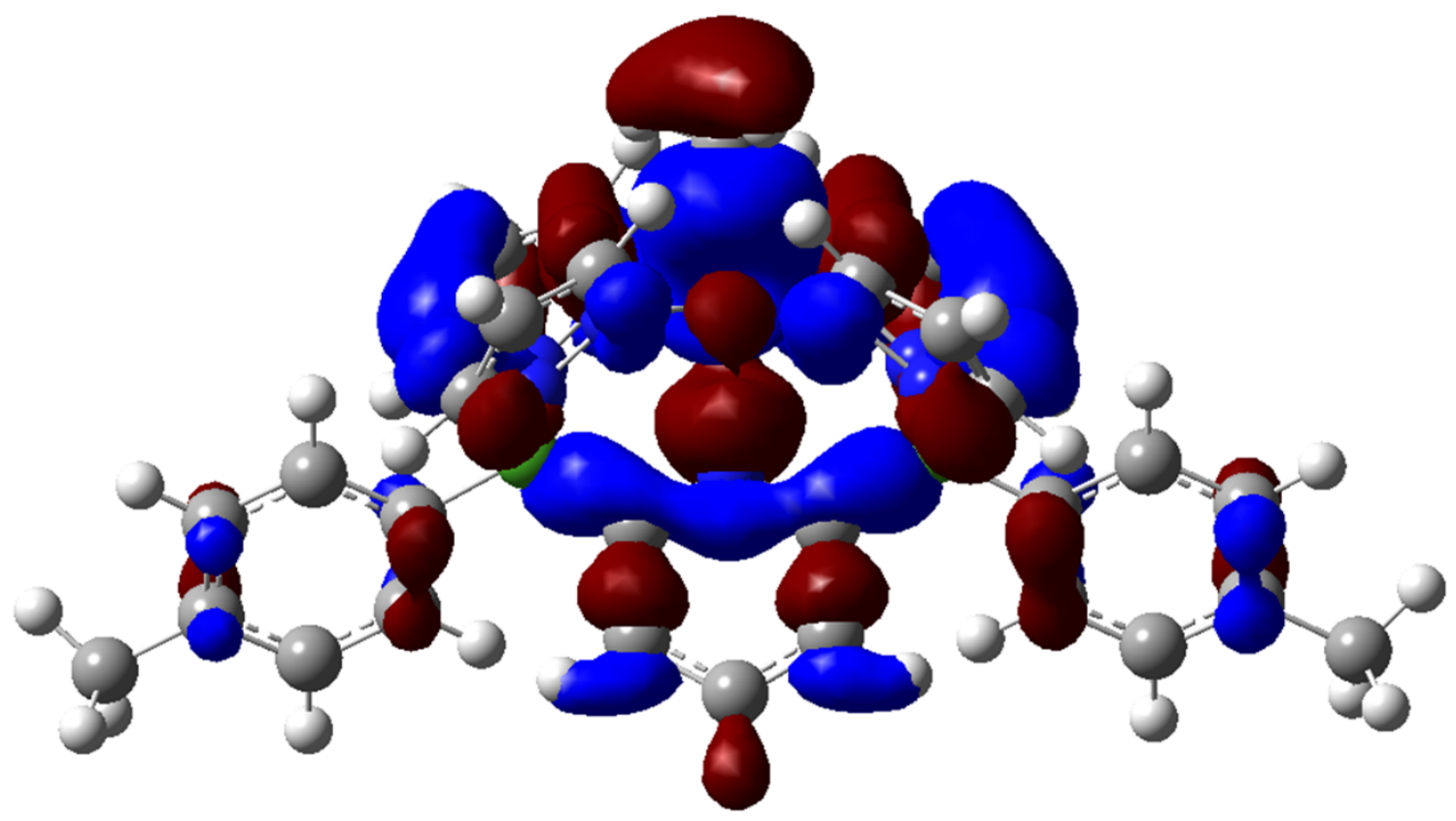

Figure S39. Depiction of the HOMO of 2-Me. 
NMR spectra of characterized compounds

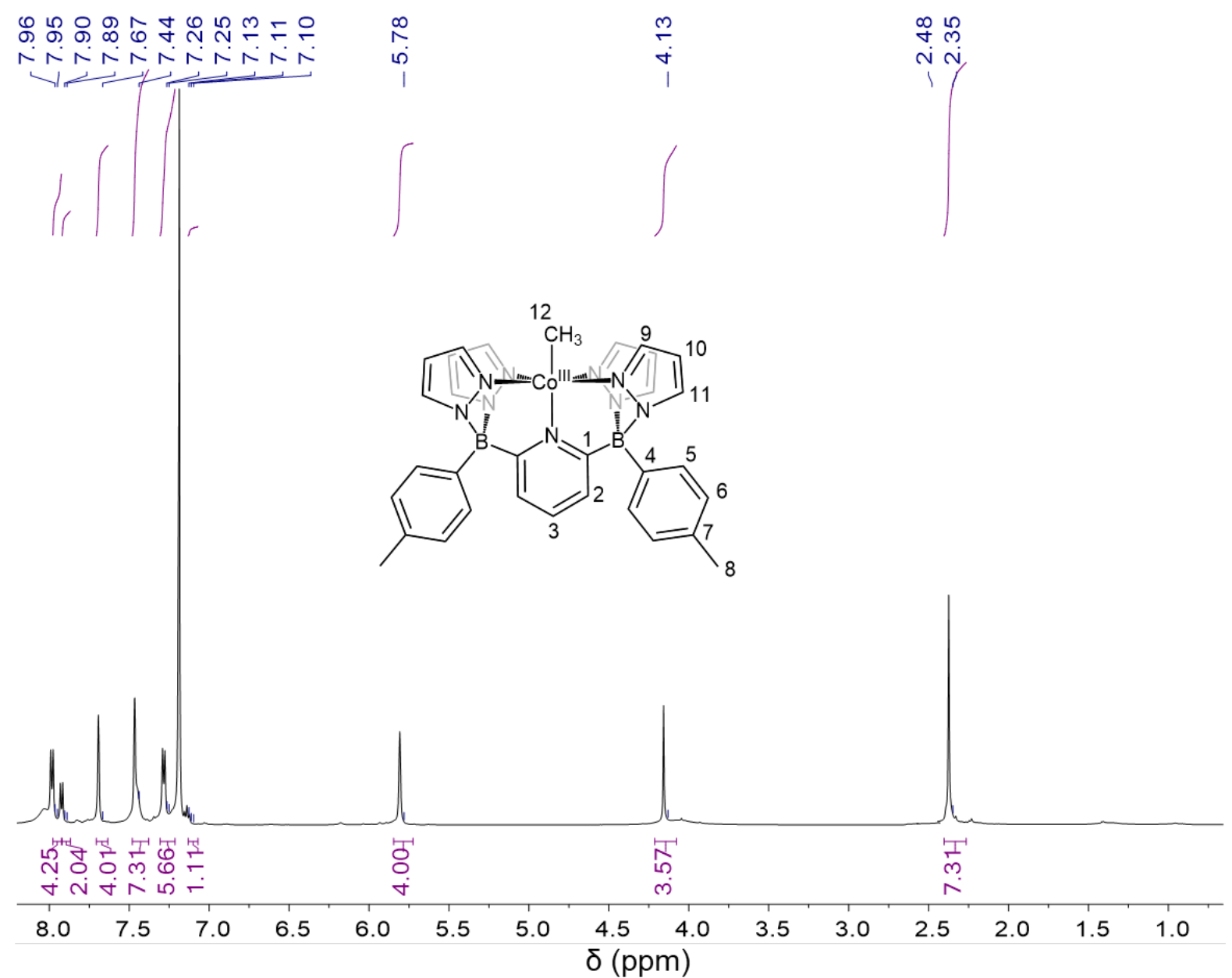

Figure S40. ${ }^{1} \mathrm{H}$ NMR spectrum of 2-Me in $\mathrm{C}_{6} \mathrm{D}_{6}$. 


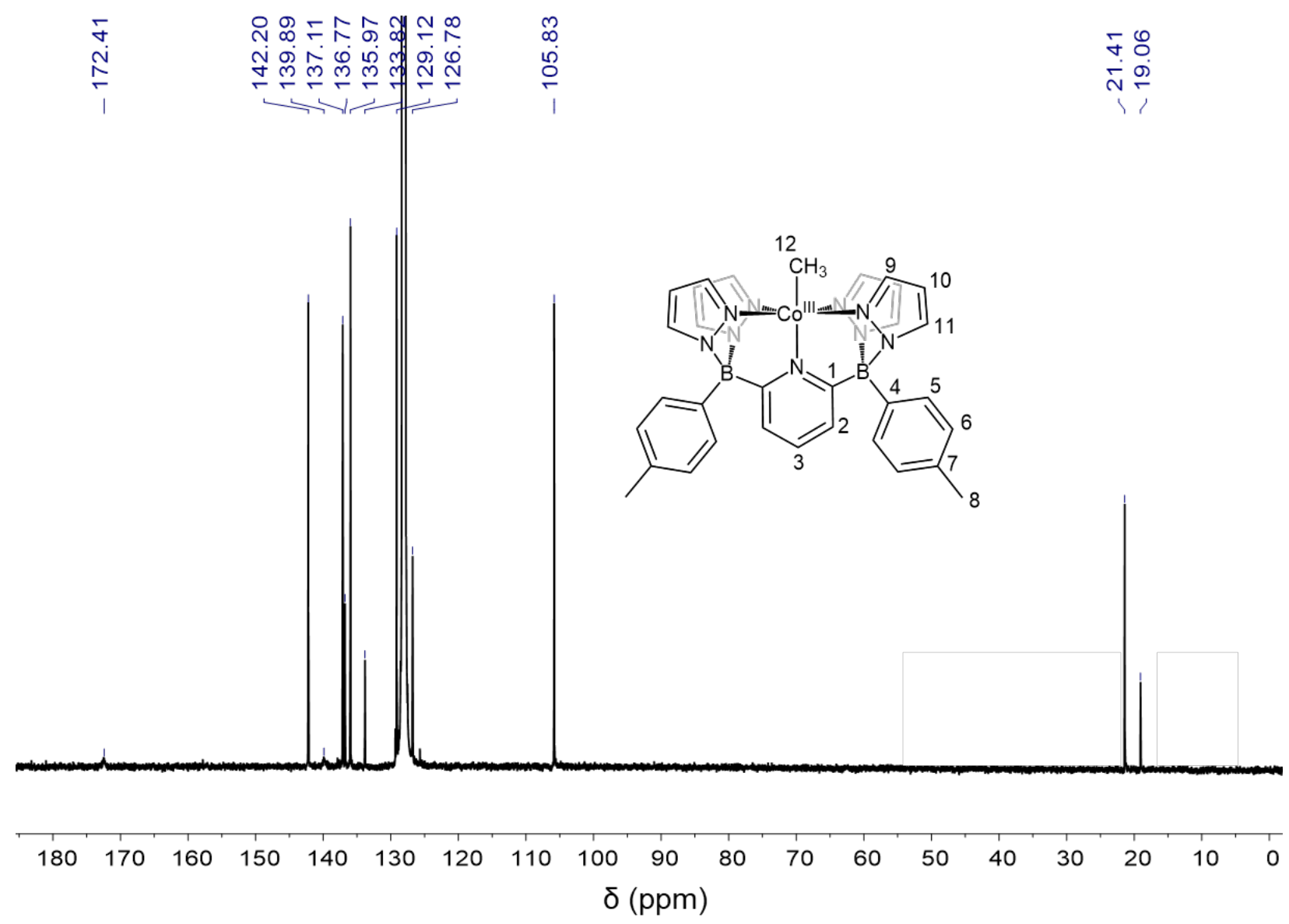

Figure S41. ${ }^{13} \mathrm{C}\left\{{ }^{1} \mathrm{H}\right\}$ NMR spectrum of 2-Me in $\mathrm{C}_{6} \mathrm{D}_{6}$. 


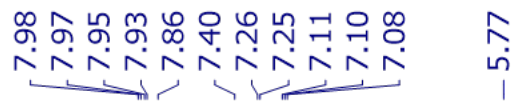

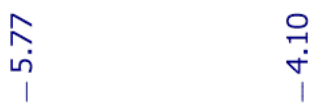

$\stackrel{\stackrel{m}{m}}{i}$

:

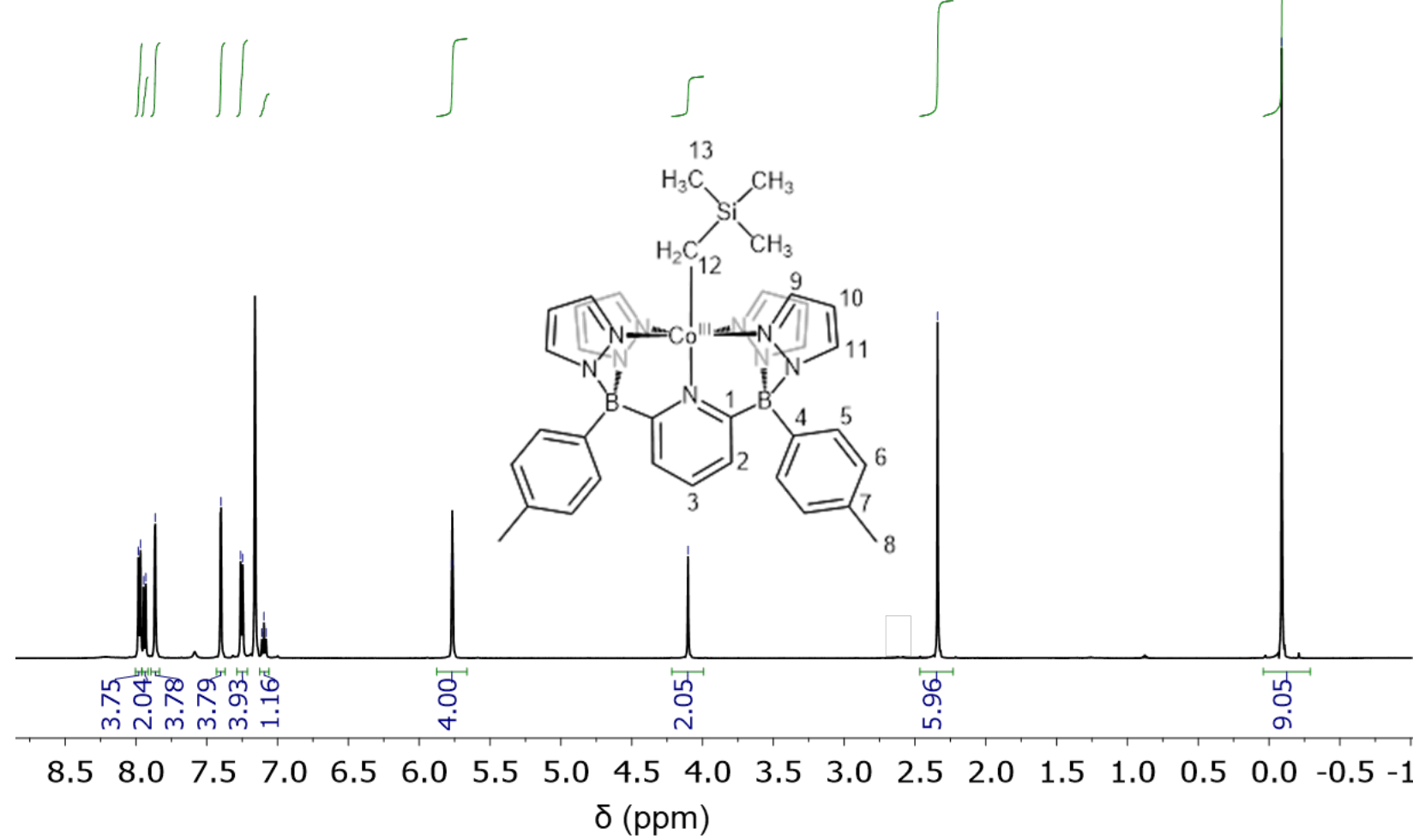

Figure S42. ${ }^{1} \mathrm{H}$ NMR spectrum of $2-\mathrm{CH}_{2} \mathrm{SiMe}_{3}$ in $\mathrm{C}_{6} \mathrm{D}_{6}$. 


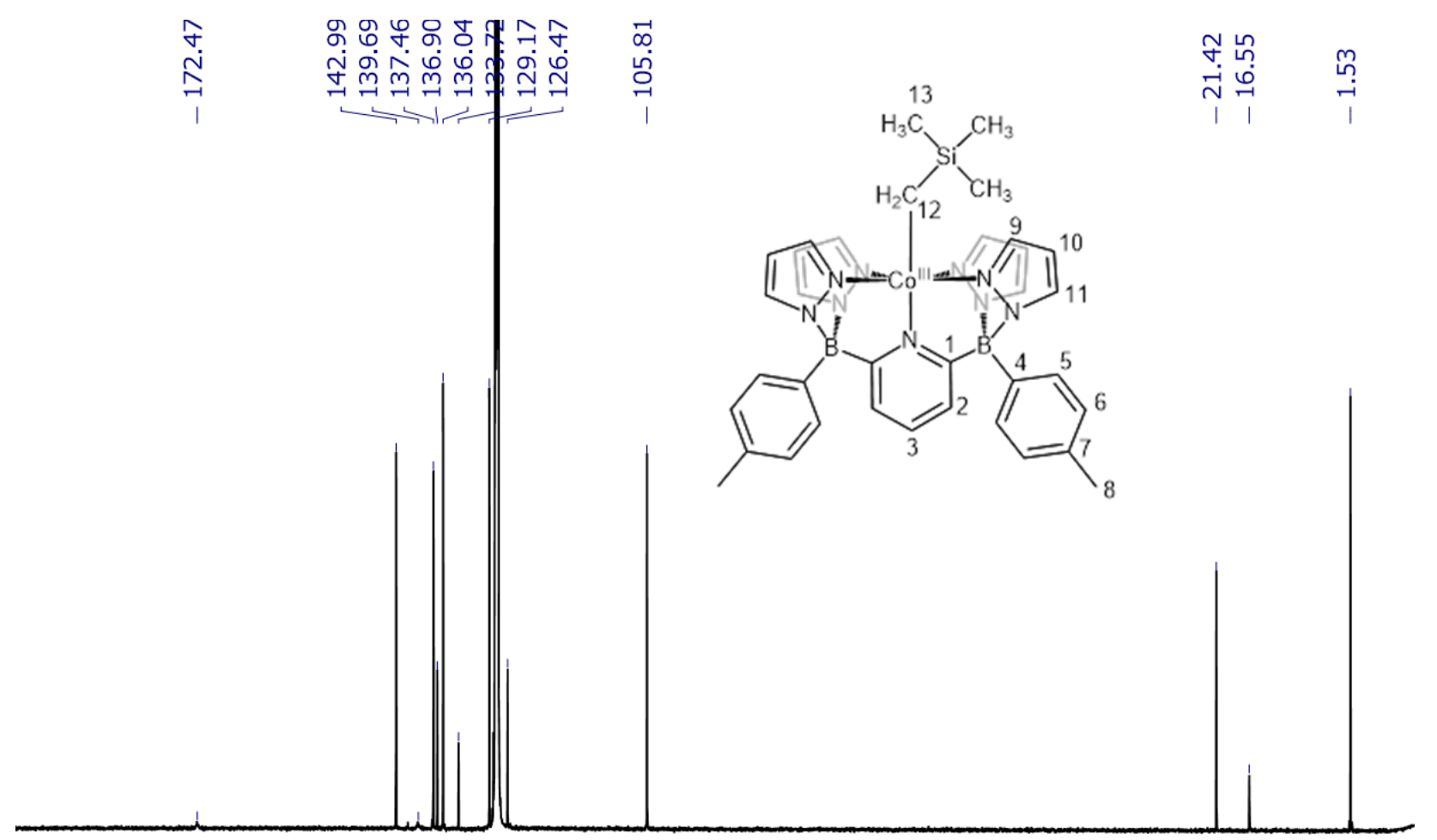

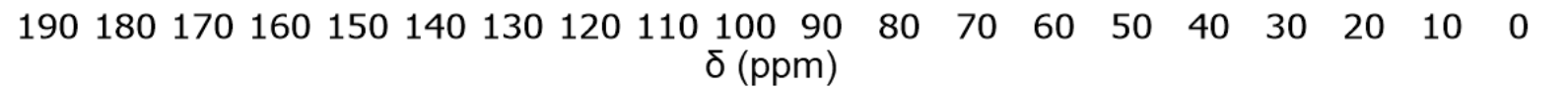

Figure S43. ${ }^{13} \mathrm{C}\left\{{ }^{1} \mathrm{H}\right\}$ NMR spectrum of $2-\mathrm{CH}_{2} \mathrm{SiMe}_{3}$ in $\mathrm{C}_{6} \mathrm{D}_{6}$. 


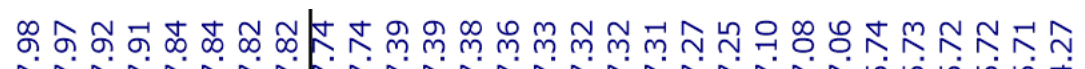

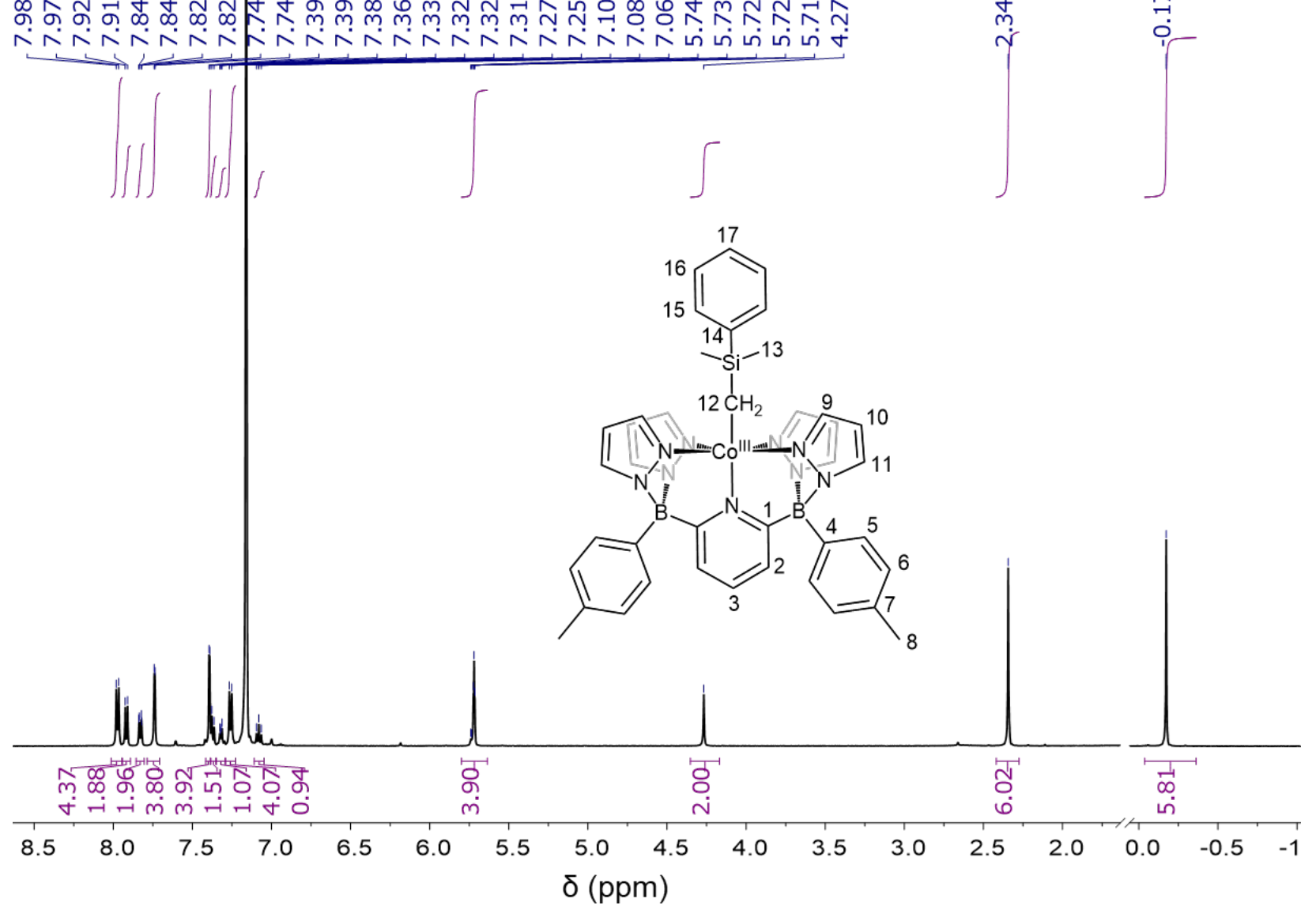

Figure S44. ${ }^{1} \mathrm{H}$ NMR spectrum of $2-\mathrm{CH}_{2} \mathrm{SiMe}_{2} \mathrm{Ph}$ in $\mathrm{C}_{6} \mathrm{D}_{6}$. 


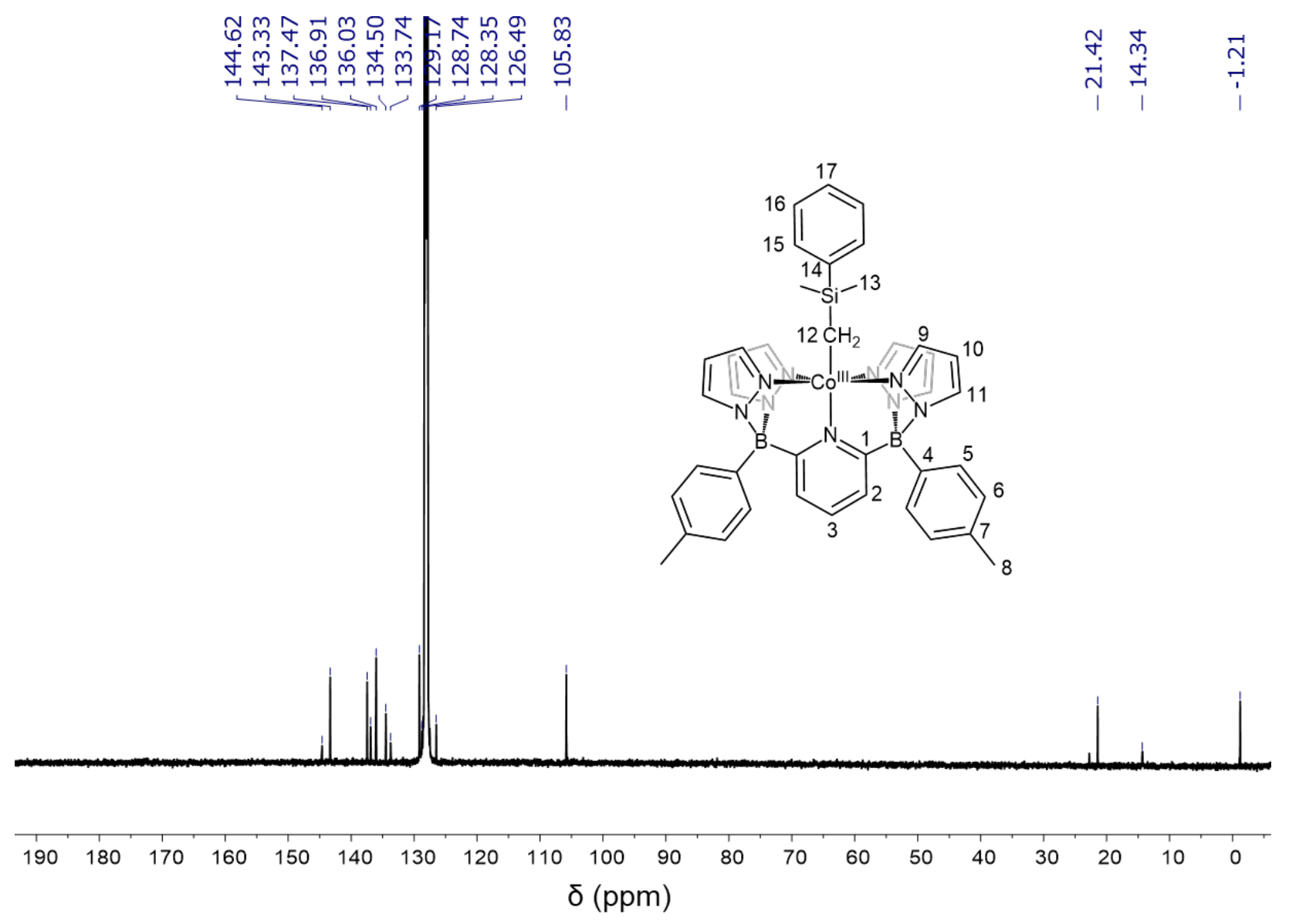

Figure S45. ${ }^{13} \mathrm{C}\left\{{ }^{1} \mathrm{H}\right\}$ NMR spectrum of $2-\mathrm{CH}_{2} \mathrm{SiMe}_{2} \mathrm{Ph}$ in $\mathrm{C}_{6} \mathrm{D}_{6}$. 


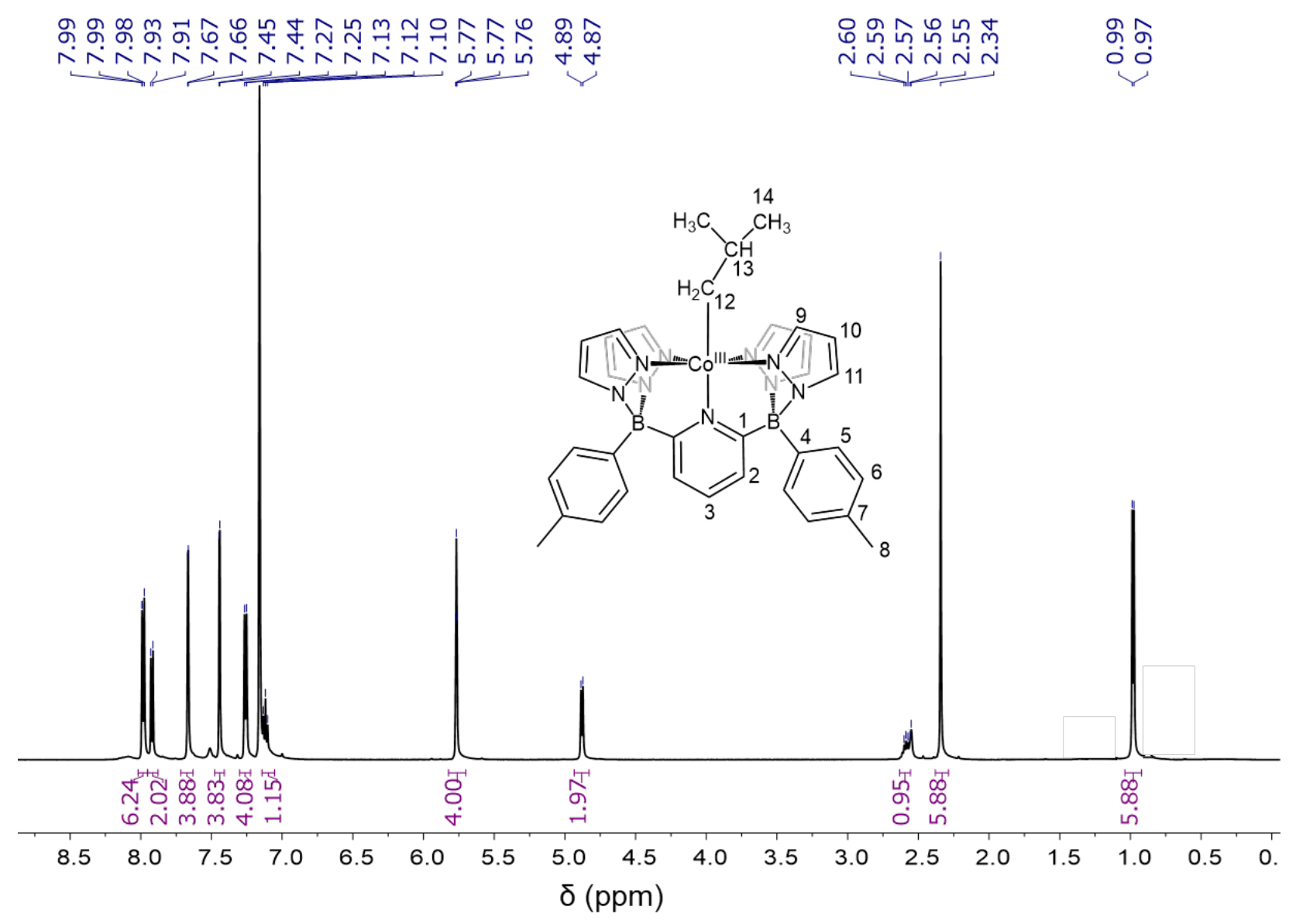

Figure S46. ${ }^{1} \mathrm{H}$ NMR spectrum of 2-'Bu in $\mathrm{C}_{6} \mathrm{D}_{6}$. 


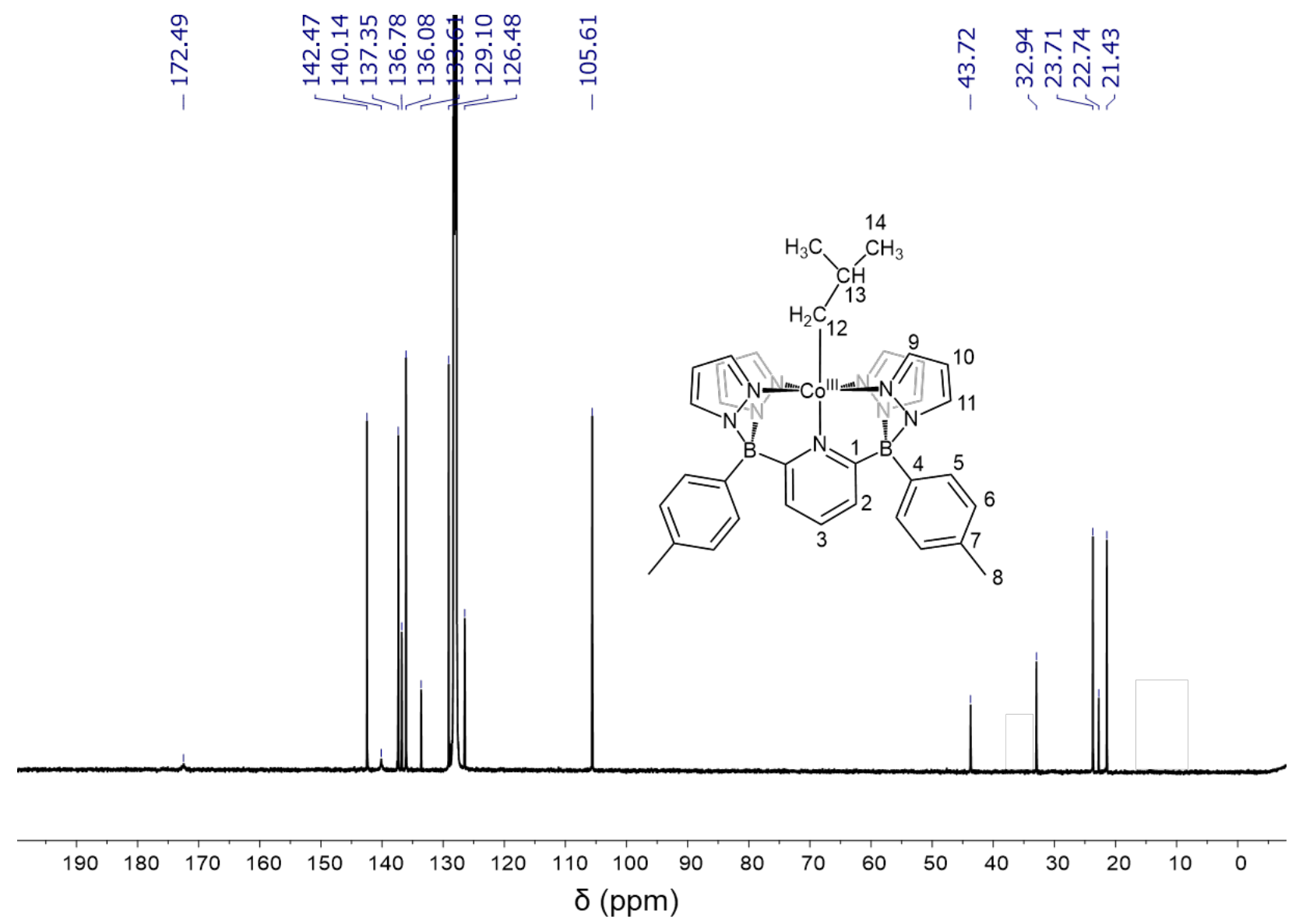

Figure S47. ${ }^{13} \mathrm{C}\left\{{ }^{1} \mathrm{H}\right\}$ NMR spectrum of 2-'Bu in $\mathrm{C}_{6} \mathrm{D}_{6}$. 


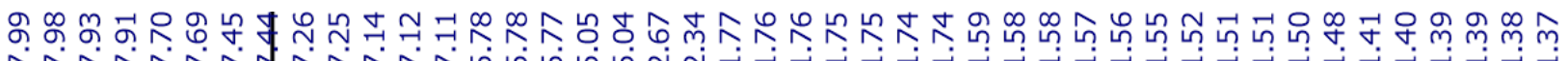

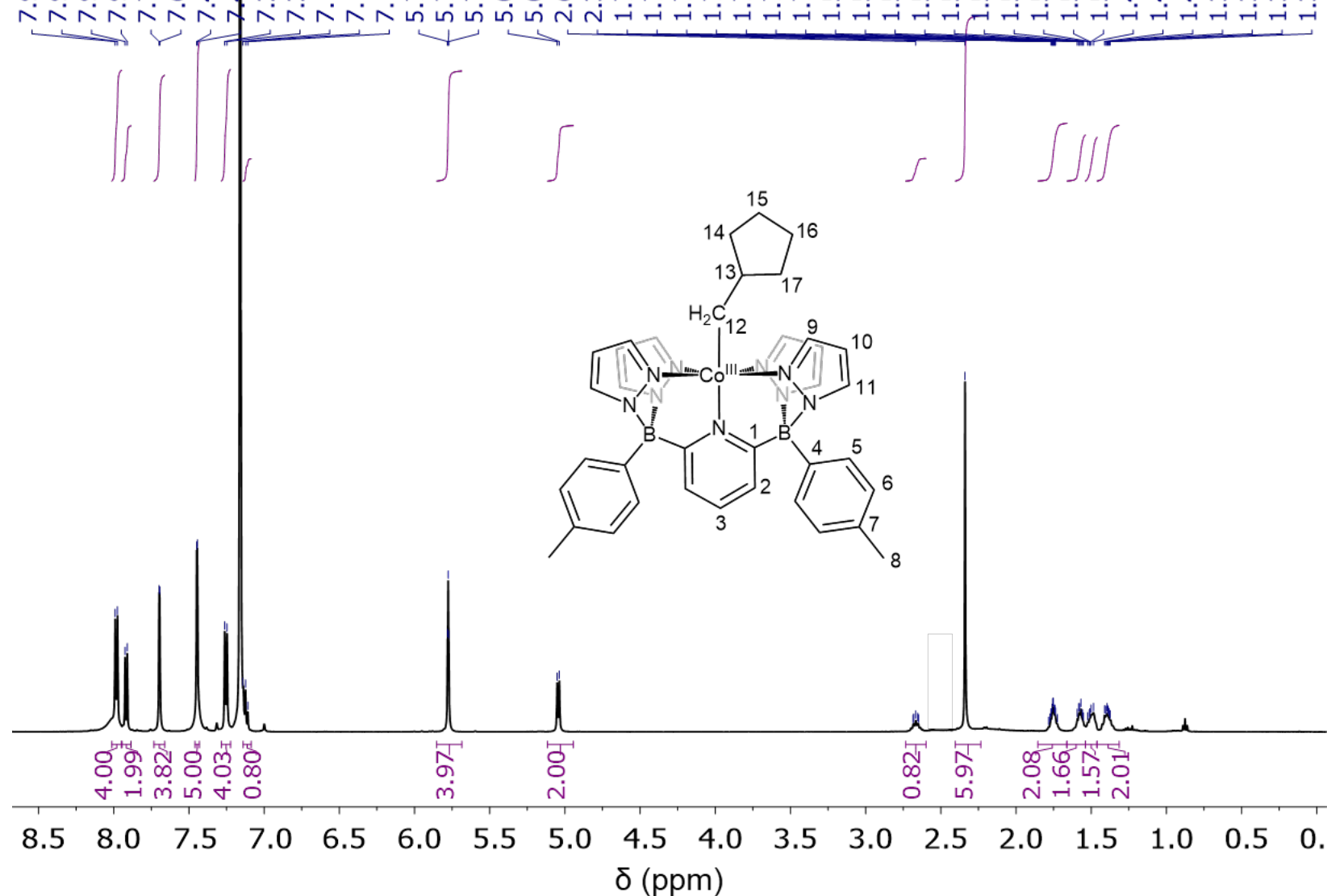

Figure S48. ${ }^{1} \mathrm{H}$ NMR spectrum of $2-\mathrm{CH}_{\mathbf{2}}\left(\boldsymbol{c}-\mathrm{C}_{5} \mathrm{H}_{9}\right)$ in $\mathrm{C}_{6} \mathrm{D}_{6}$. 


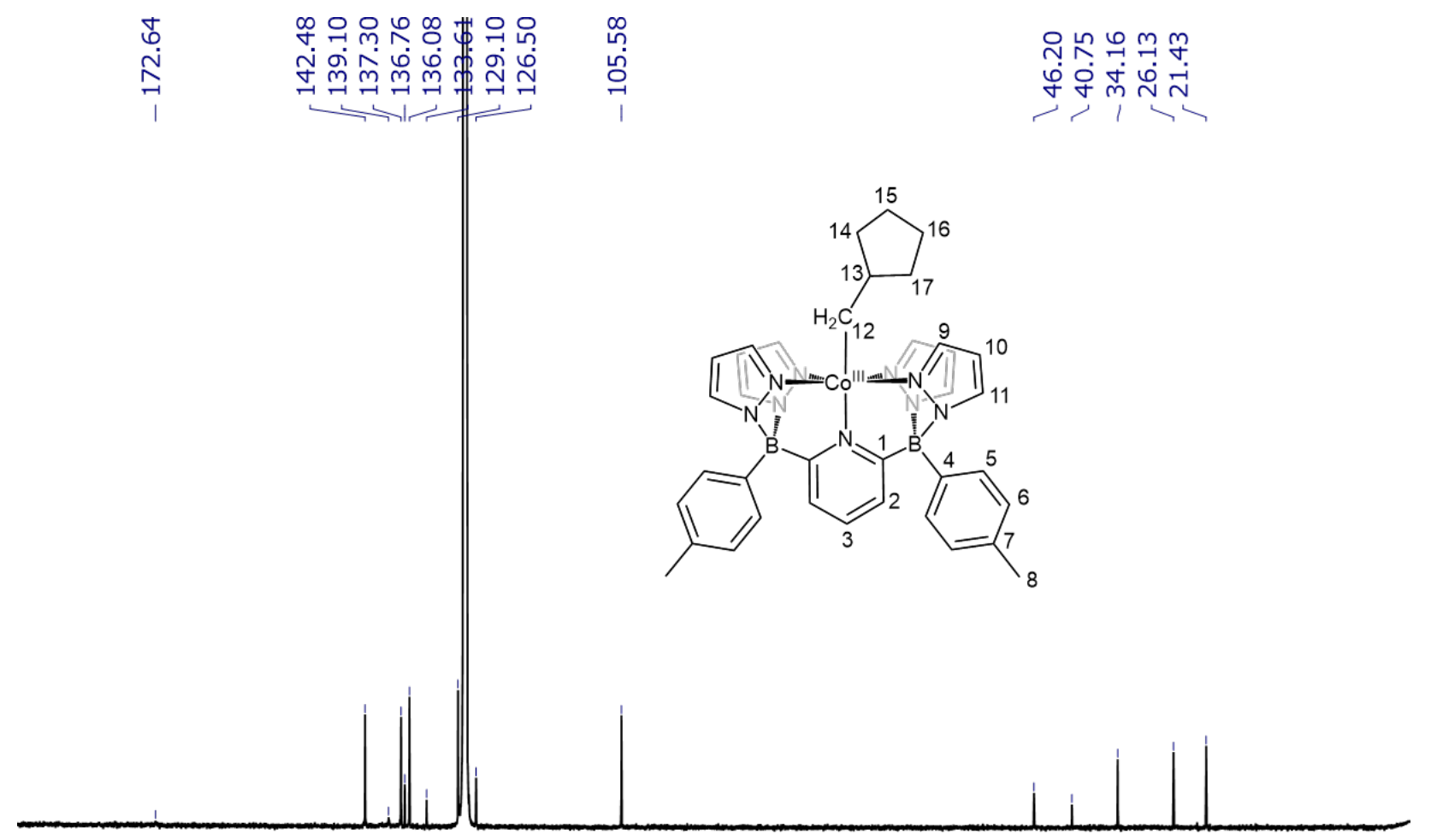

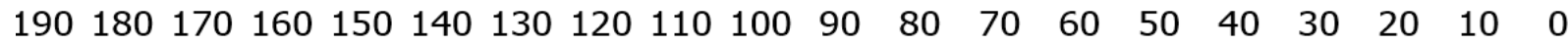
$\delta(\mathrm{ppm})$

Figure S49. ${ }^{13} \mathrm{C}\left\{{ }^{1} \mathrm{H}\right\}$ NMR spectrum of $2-\mathrm{CH}_{2}\left(\boldsymbol{c}-\mathrm{C}_{5} \mathrm{H}_{9}\right)$ in $\mathrm{C}_{6} \mathrm{D}_{6}$. 


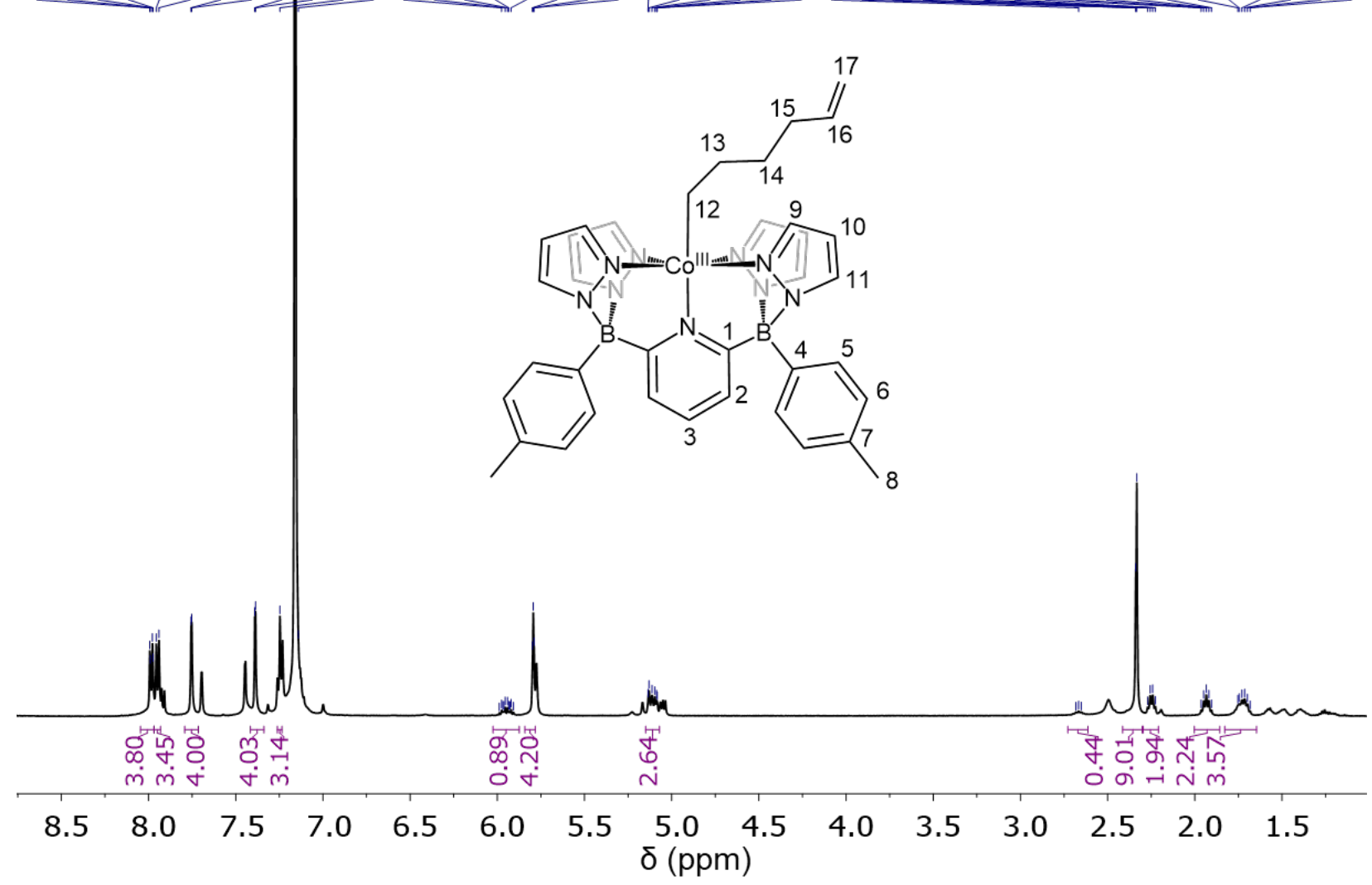

Figure S50. ${ }^{1} \mathrm{H}$ NMR spectrum of $2-\left(\mathrm{CH}_{2}\right)_{4} \mathrm{CH}=\mathrm{CH}_{2}$ in $\mathrm{C}_{6} \mathrm{D}_{6}$. 


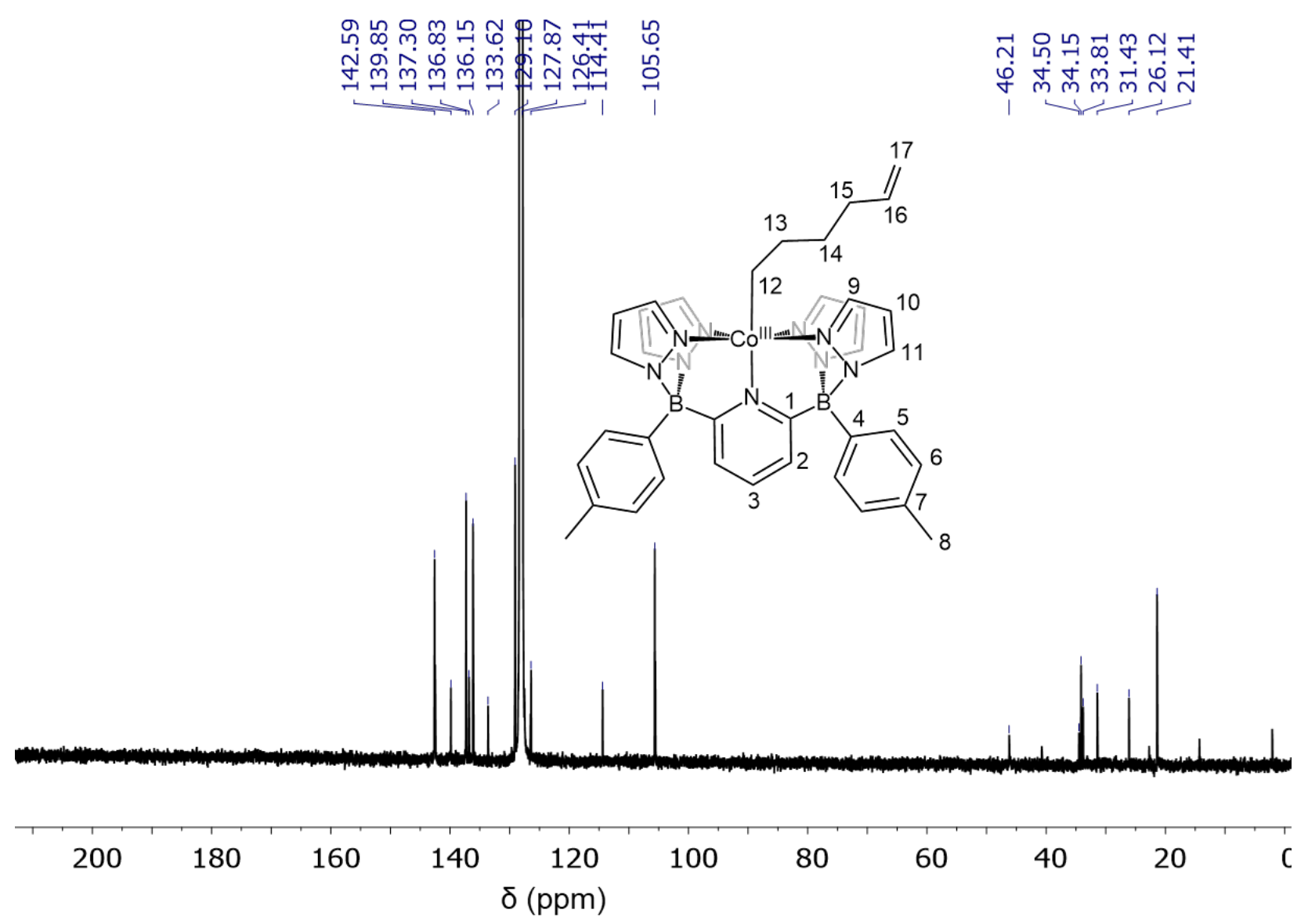

Figure S51. ${ }^{13} \mathrm{C}\left\{{ }^{1} \mathrm{H}\right\}$ NMR spectrum of 2-( $\left.\mathrm{CH}_{2}\right)_{4} \mathrm{CH}=\mathrm{CH}_{2}$ in $\mathrm{C}_{6} \mathrm{D}_{6}$. 


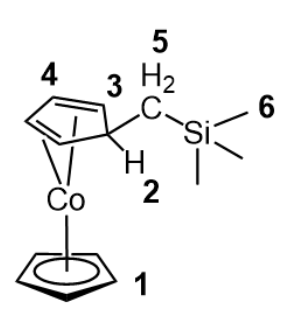

$$
\begin{array}{cc}
\text { กิ } & 0 \\
\text { ก่ } & \text { ชं }
\end{array}
$$

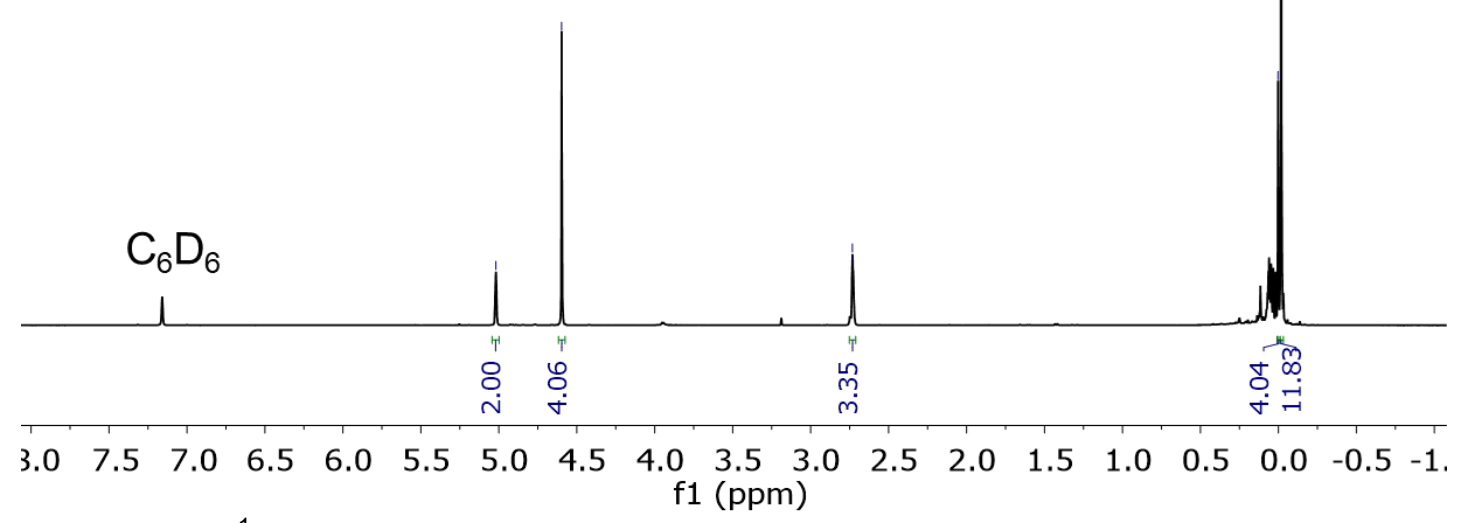

Figure S52. ${ }^{1} \mathrm{H}$ NMR spectrum (diamagnetic window) of $\mathrm{I}-\mathrm{CH}_{2} \mathrm{SiMe}_{3}$ in $\mathrm{C}_{6} \mathrm{D}_{6}$.

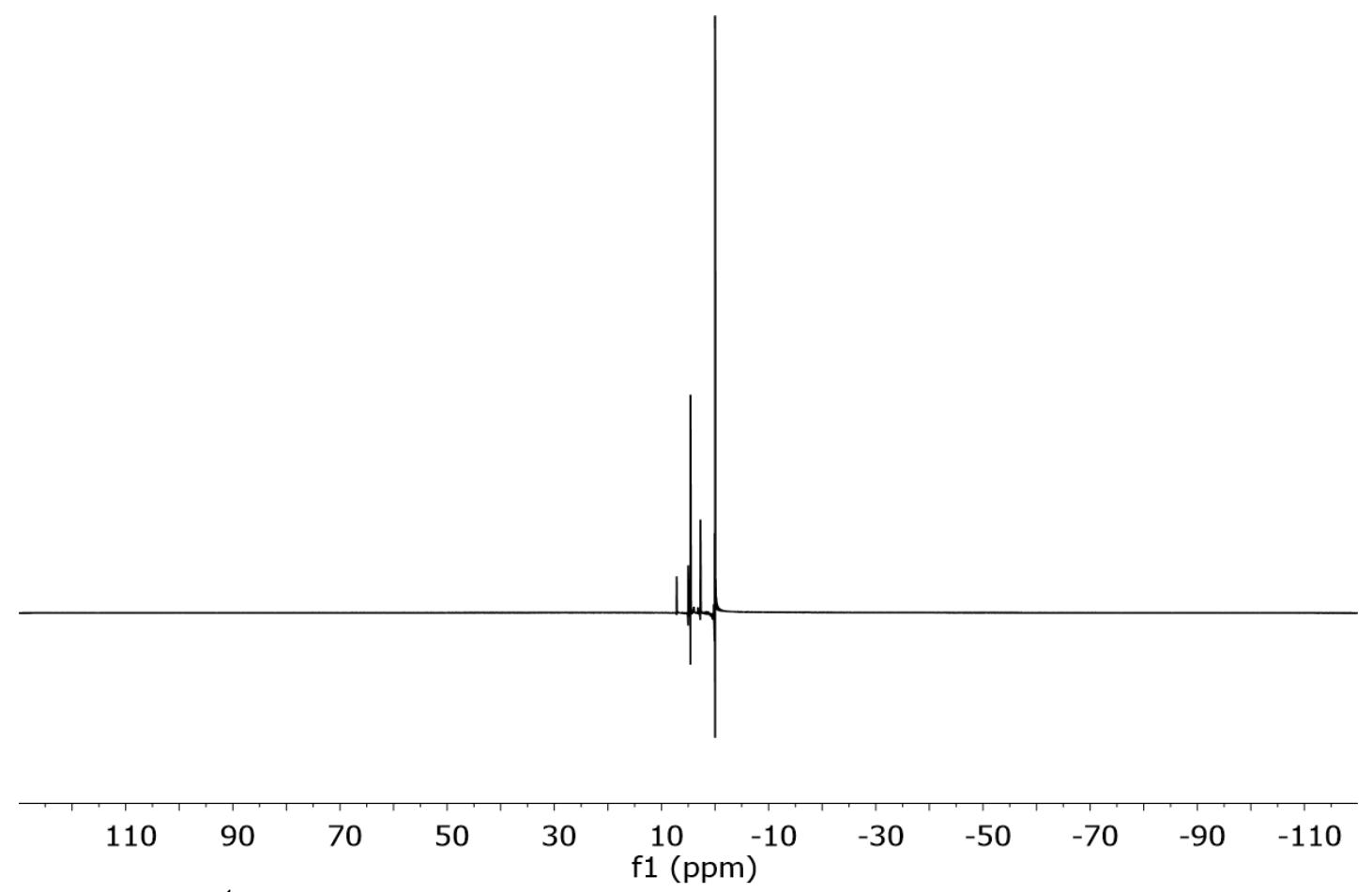

Figure S53. ${ }^{1} \mathrm{H}$ NMR spectrum (large window) of $\mathrm{I}-\mathrm{CH}_{2} \mathrm{SiMe}_{3}$ in $\mathrm{C}_{6} \mathrm{D}_{6}$. No $\mathrm{Cp}_{2} \mathrm{Co}$ is detected. 


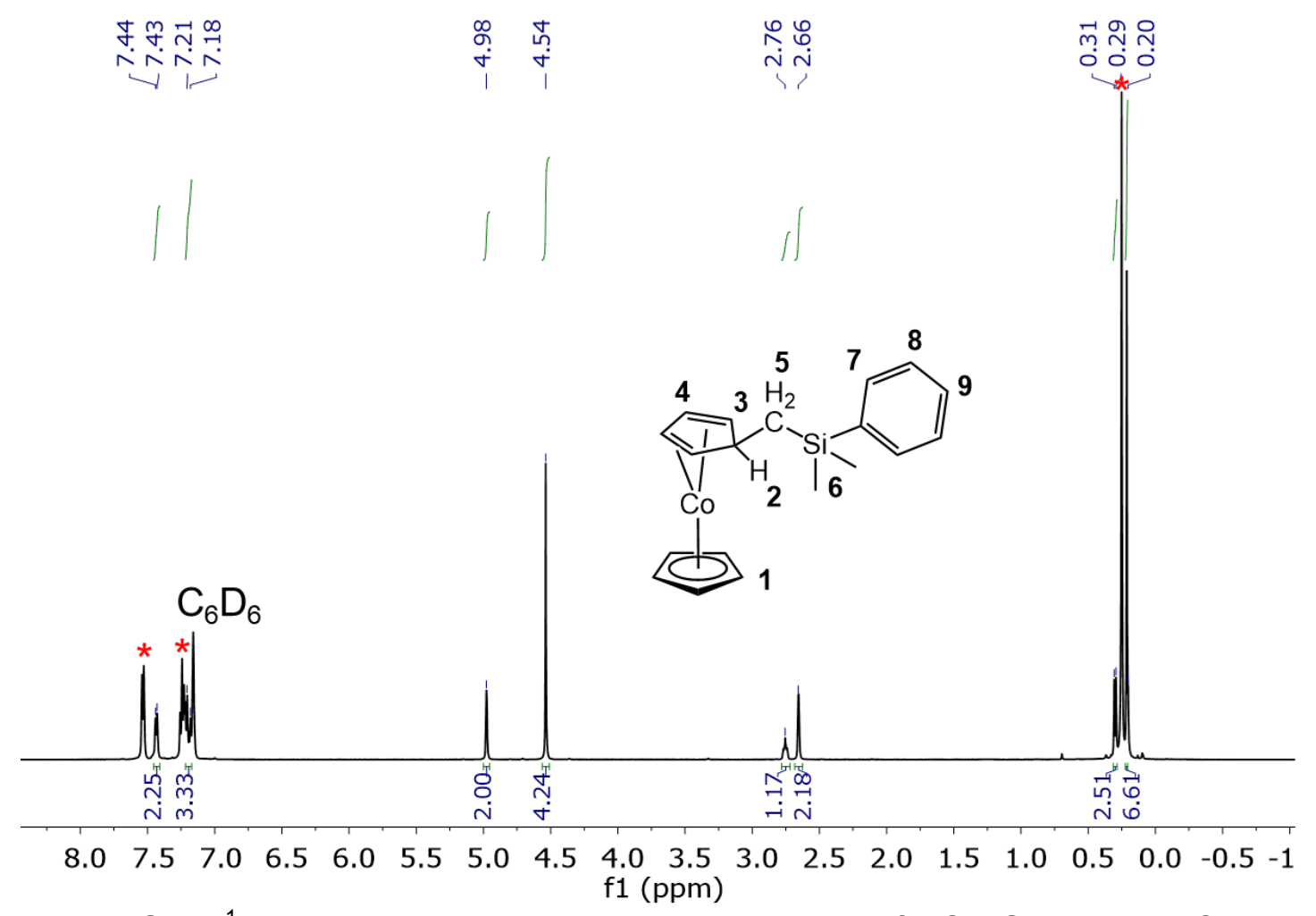

Figure S54. ${ }^{1} \mathrm{H}$ NMR spectrum (diamagnetic window) of $\mathrm{I}_{-} \mathrm{CH}_{2} \mathrm{SiMe}_{2} \mathrm{Ph}$ in $\mathrm{C}_{6} \mathrm{D}_{6}$. ${ }^{*}$ Leftover $\mathrm{LiCH}_{2} \mathrm{SiMe}_{2} \mathrm{Ph}$ due to the poor solubility of $\left[\mathrm{Cp}_{2} \mathrm{Co}\right]\left[\mathrm{PF}_{6}\right]$ in $\mathrm{C}_{6} \mathrm{D}_{6}$.

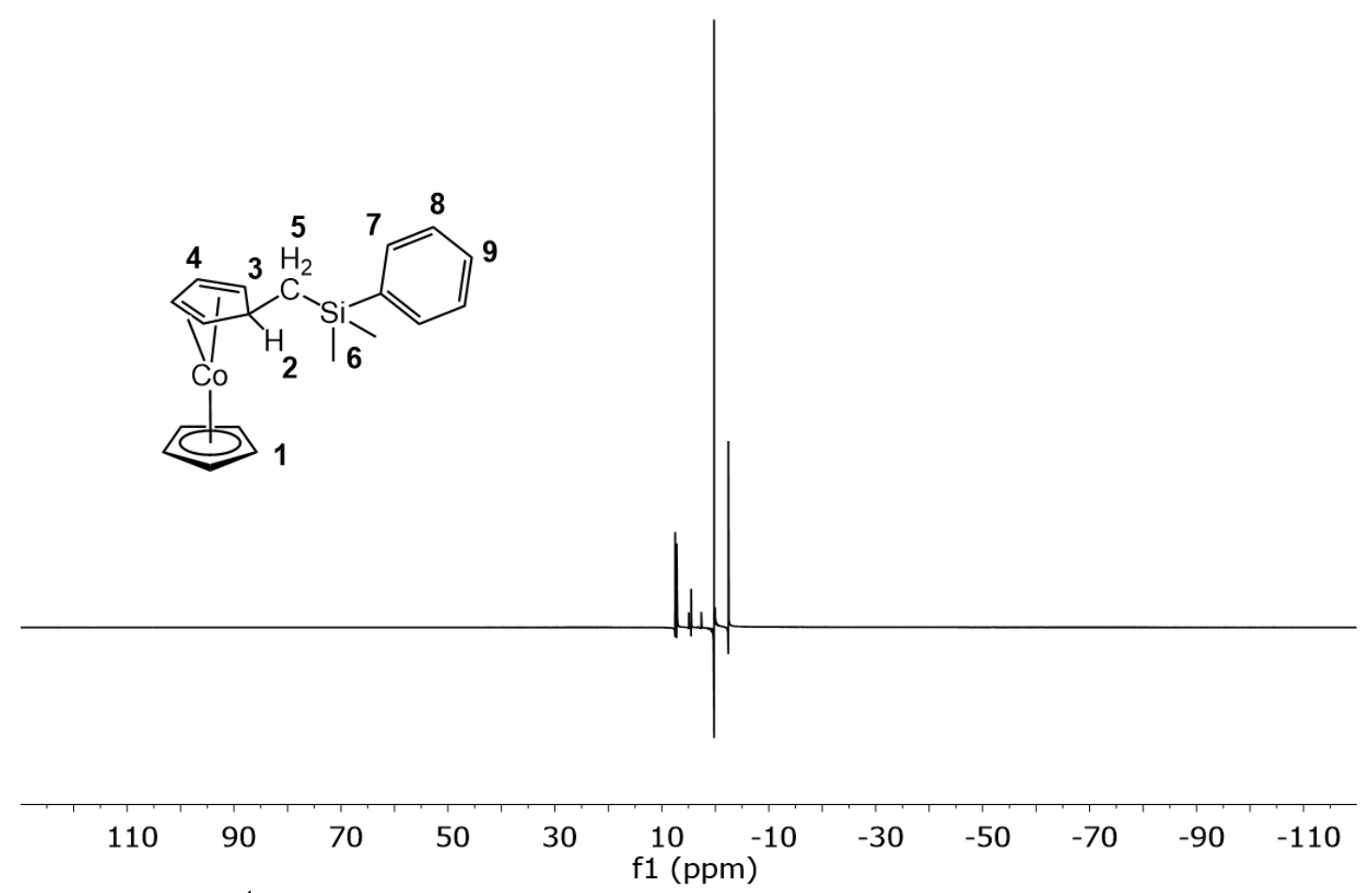

Figure S55. ${ }^{1} \mathrm{H}$ NMR spectrum (large window) of $\mathrm{I}_{-} \mathrm{CH}_{2} \mathrm{SiMe} \mathbf{e}_{2} \mathrm{Ph}$ in $\mathrm{C}_{6} \mathrm{D}_{6}$. No $\mathrm{Cp}_{2} \mathrm{Co}$ is detected. 


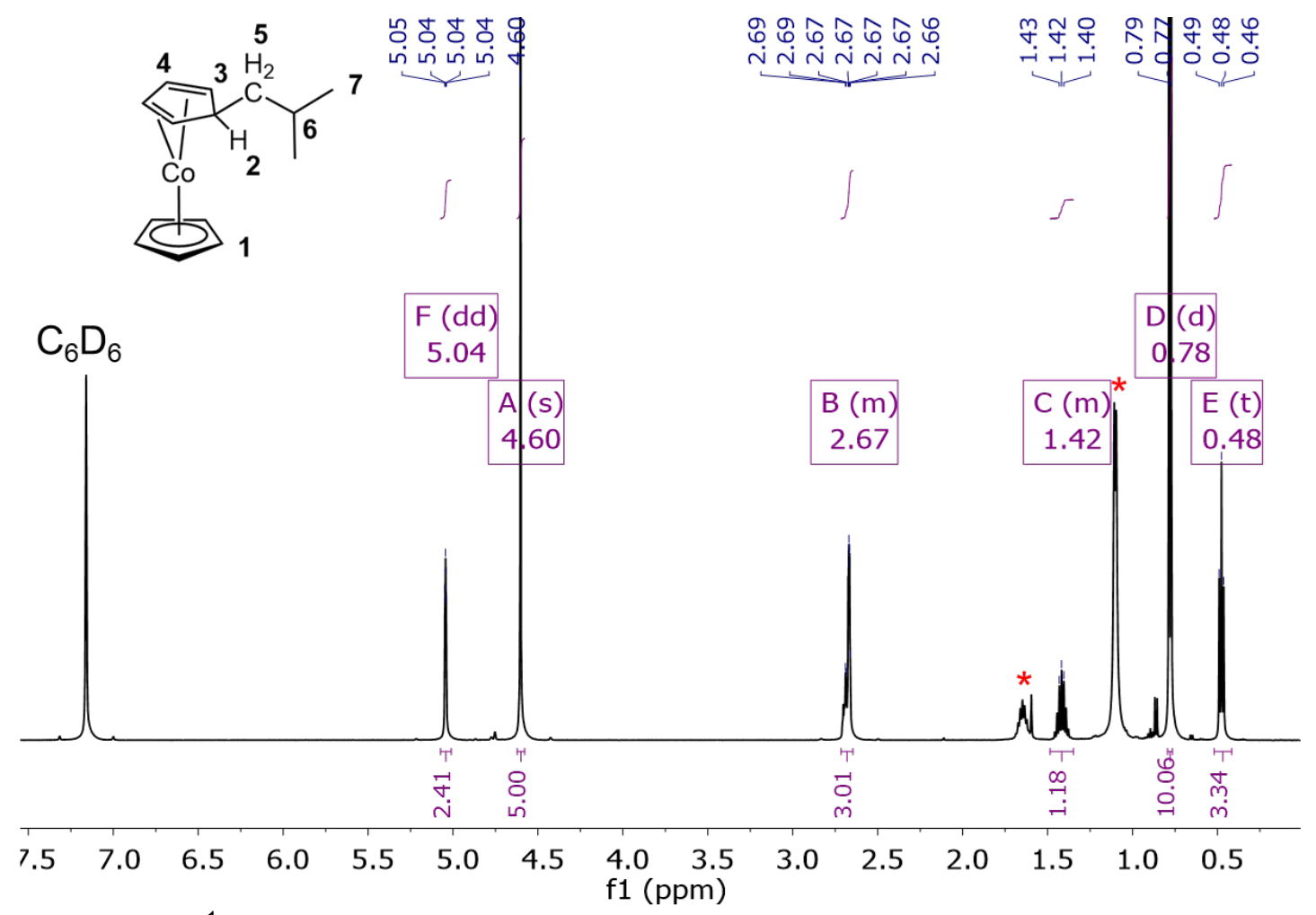

Figure S56. ${ }^{1} \mathrm{H}$ NMR spectrum (diamagnetic window) of $\mathrm{I}-\mathrm{CH}_{2} \mathrm{CH}\left(\mathrm{CH}_{3}\right)_{2}$ in $\mathrm{C}_{6} \mathrm{D}_{6}$. ${ }^{*}$ Leftover $\mathrm{LiCH} \mathrm{H}_{2} \mathrm{CH}\left(\mathrm{CH}_{3}\right)_{2}$ due to the poor solubility of $\left[\mathrm{Cp}_{2} \mathrm{Co}\right]\left[\mathrm{PF}_{6}\right]$ in $\mathrm{C}_{6} \mathrm{D}_{6}$.

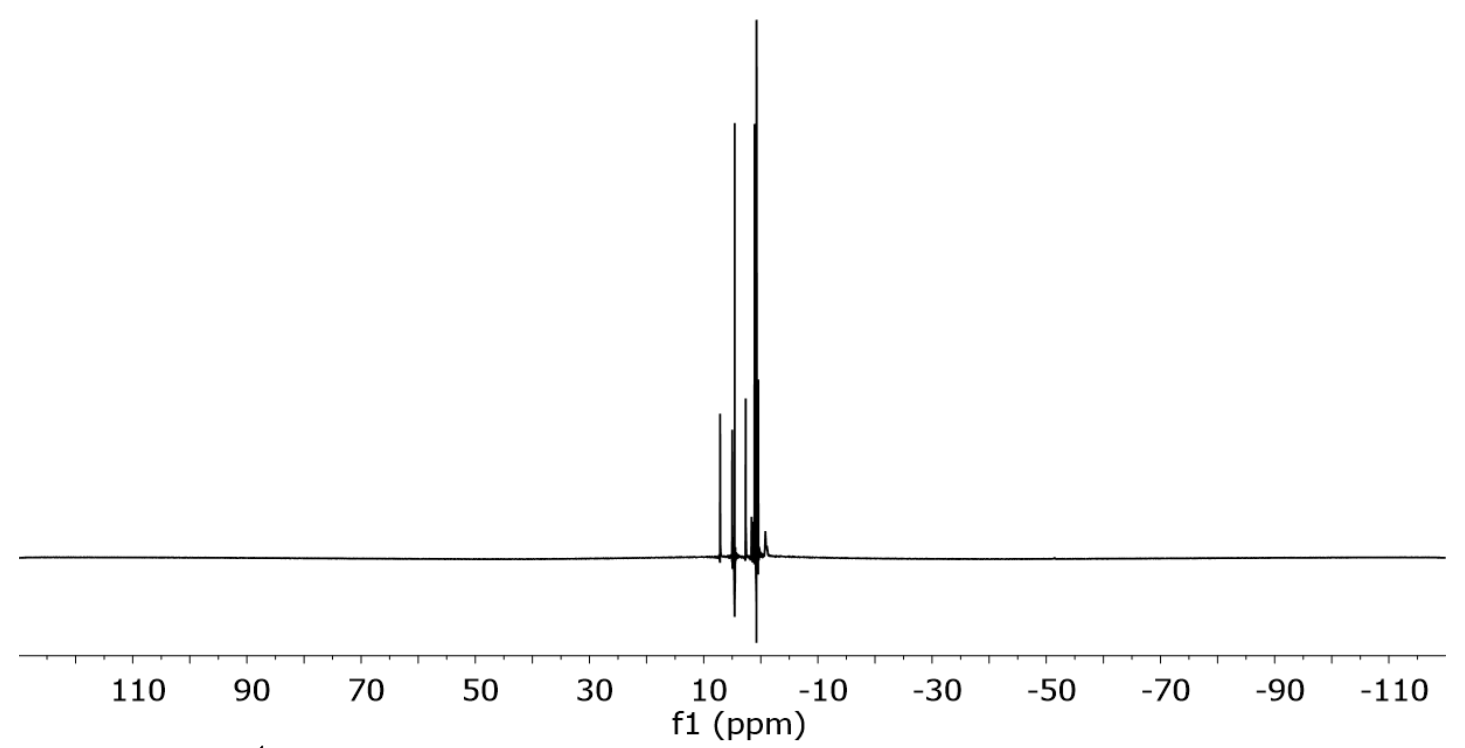

Figure S57. ${ }^{1} \mathrm{H}$ NMR spectrum (large window) of $\mathrm{I}-\mathrm{CH}_{2} \mathbf{C H}\left(\mathrm{CH}_{3}\right)_{2}$ in $\mathrm{C}_{6} \mathrm{D}_{6}$. No $\mathrm{Cp}_{2} \mathrm{Co}$ is detected. 


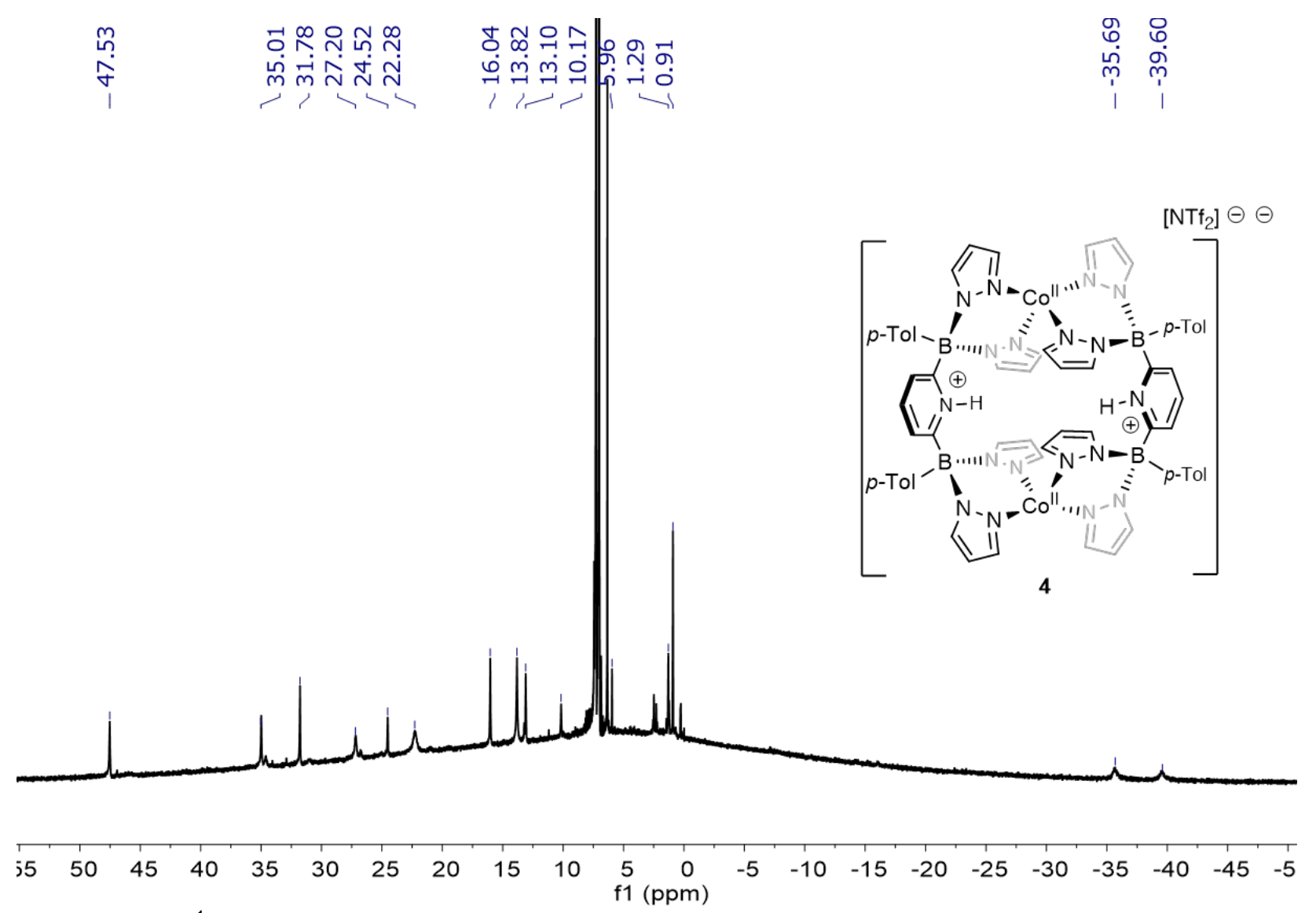

Figure S58. ${ }^{1} \mathrm{H}$ NMR spectrum of 4 in $\mathrm{o}-\mathrm{Cl}_{2}-\mathrm{C}_{6} \mathrm{D}_{4}$. 


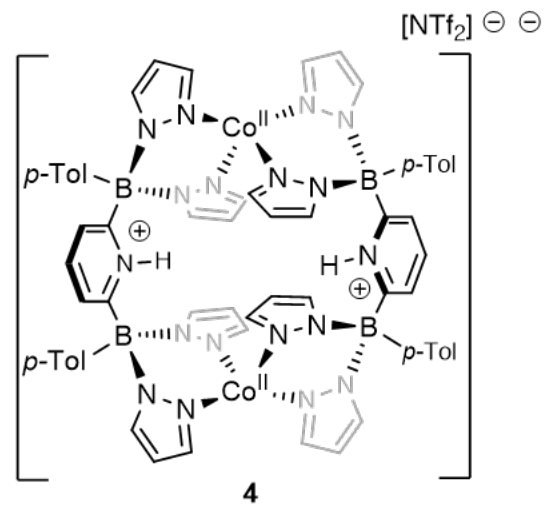

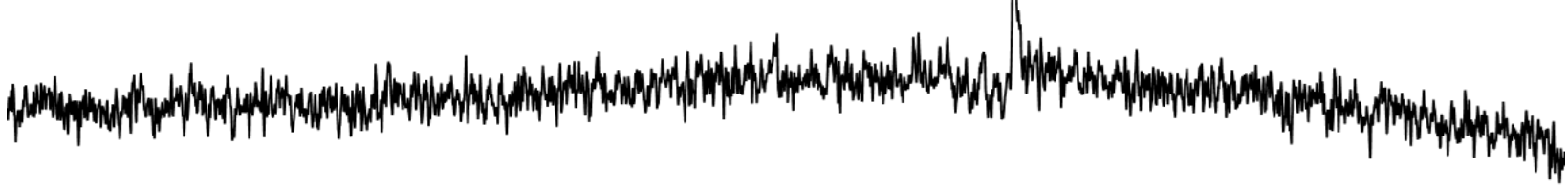

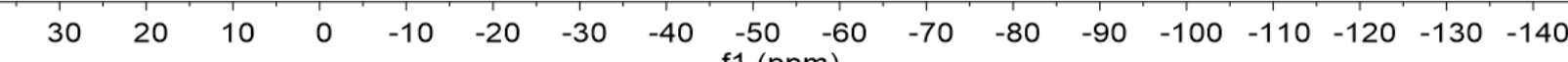
Figure S59. ${ }^{19} \mathrm{~F}$ NMR spectrum of 4 in $0-\mathrm{Cl}_{2}-\mathrm{C}_{6} \mathrm{D}_{4}$. 


\section{Additional crystallographic data of 4:}

During the refinement, it was noticed that there was a large amount of residual electron density located between the Co atoms and the ellipsoids for Co were elongated in the same direction. Given the synthetic and recrystallization conditions used, the residual electron density was attributed to bromine from bromobenzene, the recrystallization solvent. Based on the bromine present and the elongated Co ellipsoids, the Co atom was split in to Co1A and Co1B. Initially, the occupancies of bromine and Co atoms were refined independently with the occupancy refining to $0.1326(19)$ and $0.12(3)$ for $\mathrm{Br}$ and $\mathrm{Co} 1 \mathrm{~B}$, respectively. Based on their similar occupancies, Co1B is assumed be the oxidized to $\mathrm{Co}(\mathrm{III})$ and charge balanced by $\mathrm{Br}(-\mathrm{I})$. For consistency, the occupancy of $\mathrm{Br}$ and $\mathrm{Co} 1 \mathrm{~B}$ were set equal and allowed to refine, reaching occupancies of $0.1325(19)$.

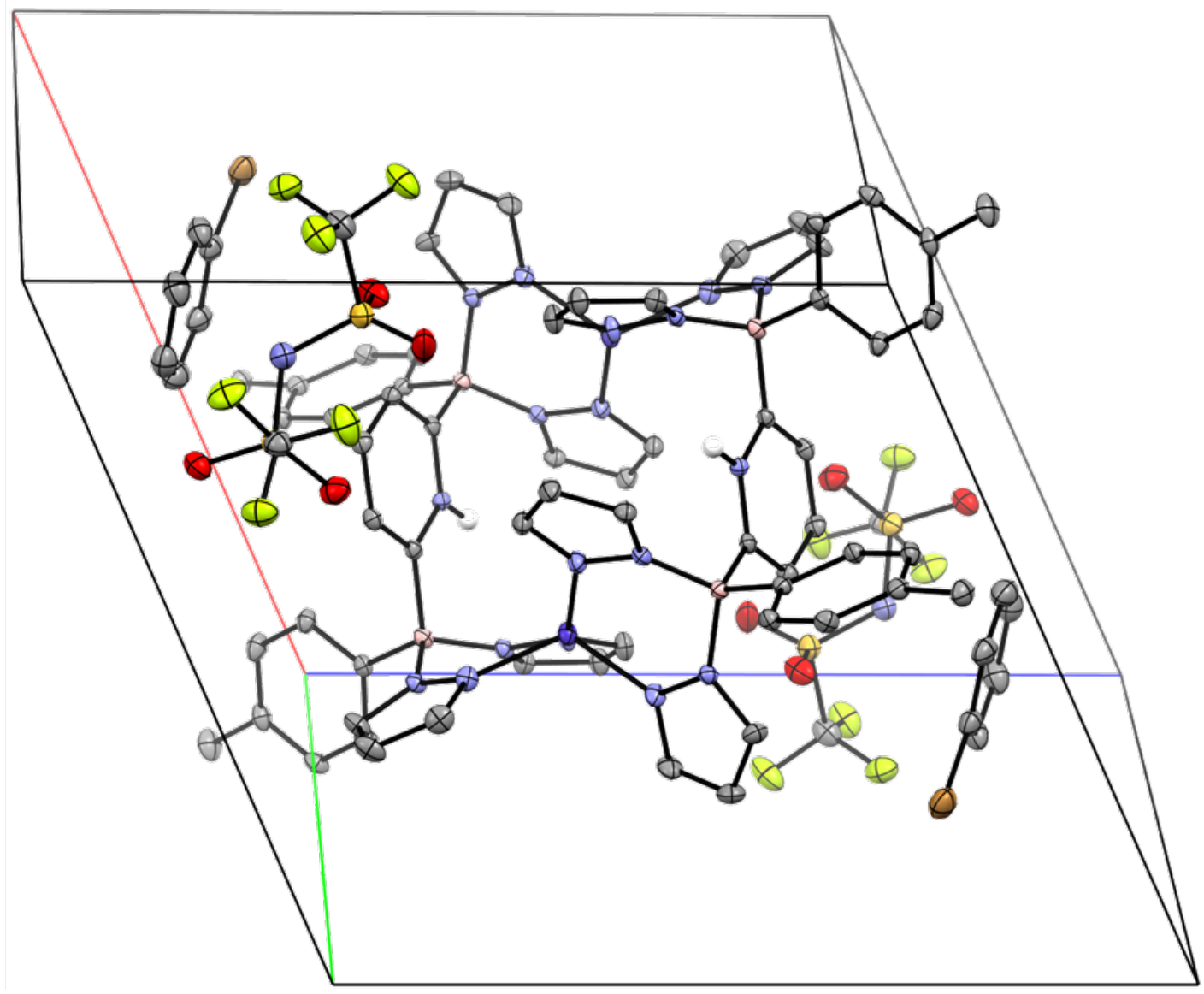

Figure S60. Unit cell contents of 4. Hydrogen, boron, carbon, nitrogen, oxygen, fluorine, sulfur, cobalt, and bromine atoms are white, pink, grey, light blue, red, green, yellow, dark blue, and gold, respectively. Ellipsoid probability set at $20 \%$. Organic hydrogen atoms and the minor components of disorder have been omitted for clarity. 


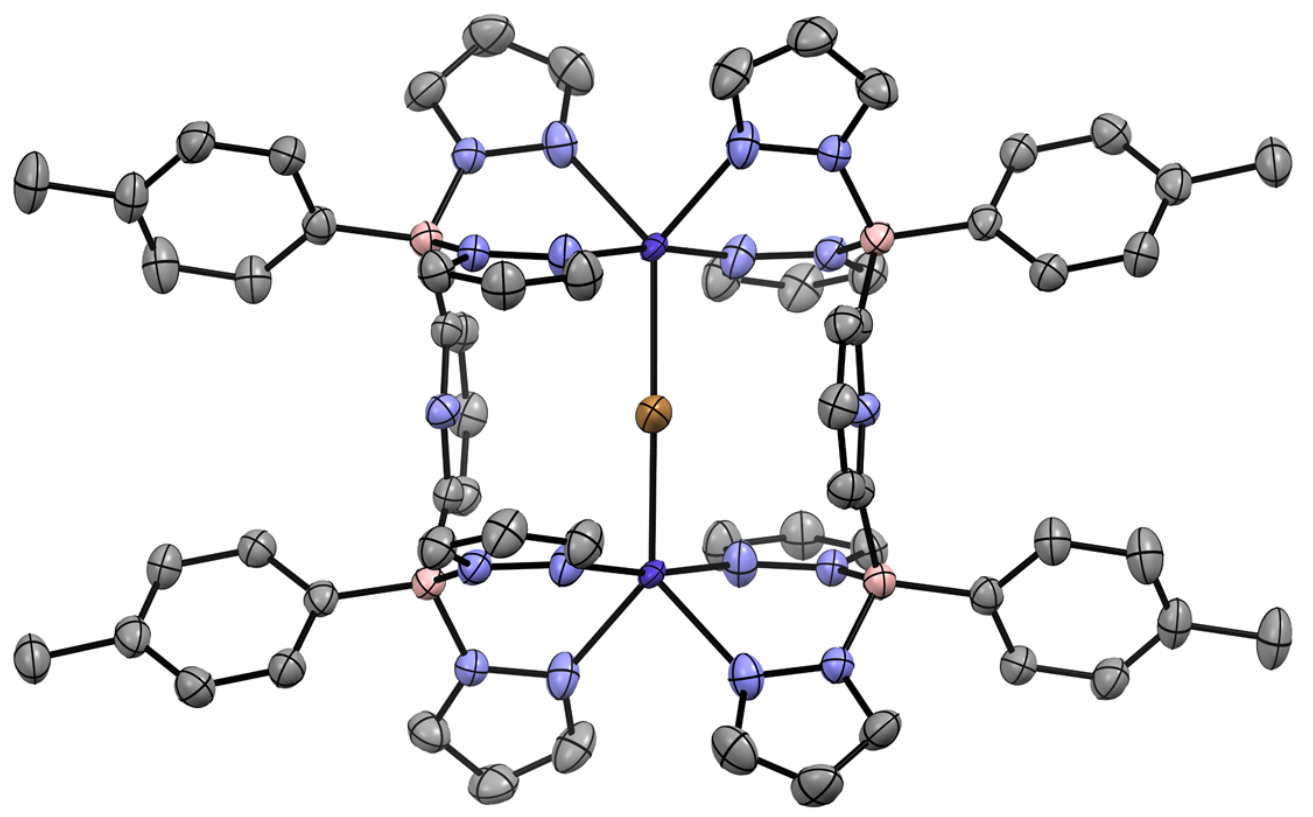

Figure S61. Structure of the Co(III) dimer present in the crystal structure of 4 due to the use of bromobenzene as the recrystallization solvent. Boron, carbon, nitrogen, cobalt, and bromine atoms are pink, grey, light blue, dark blue, and gold, respectively. Ellipsoid probability set at $50 \%$. Hydrogen atoms, one of the disordered bromine atoms, solvent molecules, and $\mathrm{NTf}_{2}$ anions have been omitted for clarity. 
Table S1

\begin{tabular}{|c|c|c|c|}
\hline & 2-Me & $2-\mathrm{CH}_{2} \mathrm{SiMe}_{3}$ & $2-\mathrm{CH}_{2} \mathrm{SiMe}_{2} \mathrm{Ph}$ \\
\hline chemical formula & $\mathrm{C}_{32} \mathrm{H}_{32} \mathrm{~B}_{2} \mathrm{CoN}_{9}$ & $\mathrm{C}_{35} \mathrm{H}_{40} \mathrm{~B}_{2} \mathrm{CoN}_{9} \mathrm{Si}$ & $\begin{array}{c}2\left(\mathrm{C}_{40} \mathrm{H}_{43} \mathrm{~B}_{2} \mathrm{CoN}_{9} \mathrm{Si}\right), \\
\mathrm{C}_{6} \mathrm{H}_{6}\end{array}$ \\
\hline crystal colour & Orange & Orange & Orange \\
\hline$F w ; F(000)$ & $623.21 ; 1296$ & $695.40 ; 2912.0$ & $1595.05,1672.0$ \\
\hline$T(\mathrm{~K})$ & $173(2)$ & $173(2)$ & $173(2)$ \\
\hline wavelength $(\AA)$ & 0.71073 & 0.71073 & 1.54178 \\
\hline space group & $P 21 / c$ & $\mathrm{C} 2 / \mathrm{c}$ & $P-1$ \\
\hline$a(\AA)$ & 20.0211)17) & $39.0465(14)$ & $15.0959(5)$ \\
\hline$b(\AA)$ & $17.4573(11)$ & $8.3423(2)$ & $17.0864(5)$ \\
\hline$c(\AA)$ & $8.3874(6)$ & $26.7224(10)$ & $17.5752(5)$ \\
\hline$\alpha(\operatorname{deg})$ & 90 & 90 & $107.220(2)$ \\
\hline$\beta$ (deg) & $97.808(5)$ & $126.921(2)$ & $94.154(2)$ \\
\hline Y (deg) & 90 & 90 & $109.589(2)$ \\
\hline$Z$ & 4 & 8 & 2 \\
\hline$V\left(\AA^{3}\right)$ & $2904.3(4)$ & $6958.9(4)$ & $4004.5(2)$ \\
\hline$\rho_{\text {calcd }}\left(\mathbf{g} \cdot \mathrm{cm}^{-3}\right)$ & 1.425 & 1.327 & 1.323 \\
\hline$\mu\left(\mathrm{mm}^{-1}\right)$ & 0.632 & 0.568 & 3.986 \\
\hline $\begin{array}{l}\theta \text { range }(\mathrm{deg}) ; \\
\text { completeness }\end{array}$ & $\begin{array}{c}2.549-25.999 \\
0.993\end{array}$ & $\begin{array}{c}2.991-27.512 \\
0.996\end{array}$ & $\begin{array}{c}2.681-72.392 \\
0.957\end{array}$ \\
\hline $\begin{array}{c}\text { collected reflections; } \\
\mathbf{R}_{\sigma}\end{array}$ & $5679 ; 0.0548$ & $7969 ; 0.1003$ & $15179 ; 0.0287$ \\
\hline \begin{tabular}{|c|} 
unique reflections; \\
$\mathbf{R}_{\text {int }}$
\end{tabular} & $3259 ; 0.1146$ & $4761 ; 0.1278$ & $7546 ; 0.0512$ \\
\hline$R 1^{a} ; w R 2^{b}[I>2 \sigma(I)]$ & $0.0548 ; 0.0990$ & $0.0579 ; 0.1210$ & $0.2429 ; 0.3112$ \\
\hline R1; wR2 [all data] & $0.1223 ; 0.1220$ & $0.1204 ; 0.1471$ & $0.1314 ; 0.4058$ \\
\hline GOF & 1.005 & 1.012 & 1.001 \\
\hline $\begin{array}{c}\text { largest diff peak and } \\
\text { hole }\end{array}$ & 0.320 and -0.491 & 0.456 and -0.522 & 0.889 and -0.707 \\
\hline
\end{tabular}

${ }^{\mathrm{a}} \boldsymbol{R}_{\mathbf{1}}=\boldsymbol{\Sigma}\left(|| \mathrm{F}_{\mathrm{o}}|-| \mathrm{F}_{\mathrm{c}}||\right) / \boldsymbol{\Sigma}\left|\mathrm{F}_{\mathrm{o}}\right|$

${ }^{b} w R_{2}=\left\{\Sigma\left[w\left(F_{o}^{2}-F_{c}{ }^{2}\right)^{2}\right] / \Sigma\left[w\left(F_{o}{ }^{2}\right)^{2}\right]\right\}^{1 / 2}$ 
Table S2

\begin{tabular}{|c|c|}
\hline & 4 \\
\hline $\begin{array}{l}\text { chemical } \\
\text { formula }\end{array}$ & $\mathrm{C}_{78} \mathrm{H}_{70} \mathrm{~B}_{4} \mathrm{Br}_{2.27} \mathrm{Co}_{2} \mathrm{~F}_{12} \mathrm{~N}_{20} \mathrm{O}_{8} \mathrm{~S}_{4}$ \\
\hline crystal colour & Pink \\
\hline$F w ; F(000)$ & $2114.27 ; 1067.0$ \\
\hline$T(\mathrm{~K})$ & 173.0 \\
\hline $\begin{array}{c}\text { wavelength } \\
(\AA)\end{array}$ & 1.54178 \\
\hline space group & $P-1$ \\
\hline$a(\AA)$ & $13.3026(2)$ \\
\hline$b(\AA)$ & $13.3777(2)$ \\
\hline$c(\AA)$ & $15.0410(3)$ \\
\hline$\alpha($ deg) & $90.2900(10)$ \\
\hline$\beta$ (deg) & $113.8510(10)$ \\
\hline Y (deg) & $113.6130(10)$ \\
\hline $\mathbf{Z}$ & 1 \\
\hline$V\left(\AA^{3}\right)$ & $2198.10(7)$ \\
\hline$\rho_{\text {calcd }}\left(\mathrm{g} \cdot \mathrm{cm}^{-3}\right)$ & 1.597 \\
\hline$\mu\left(\mathrm{mm}^{-1}\right)$ & 5.849 \\
\hline \begin{tabular}{|l|}
$\theta$ range $(\mathrm{deg}) ;$ \\
completeness
\end{tabular} & $6.556-130.454 ; 0.994$ \\
\hline \begin{tabular}{c|} 
collected \\
reflections; $\mathbf{R}_{\sigma}$
\end{tabular} & $7496 ; 0.0311$ \\
\hline \begin{tabular}{|c|} 
unique \\
reflections; \\
$\mathbf{R}_{\text {int }}$ \\
\end{tabular} & $7496 ; 0.0440$ \\
\hline $\begin{array}{c}\mathrm{R}^{\mathrm{a}} ; \mathrm{wR2}^{\mathrm{b}}[\mathrm{I}> \\
2 \sigma(\mathrm{I})]\end{array}$ & $0.0598 ; 0.1718$ \\
\hline $\begin{array}{c}\mathrm{R} 1 ; \text { wR2 [all } \\
\text { data] }\end{array}$ & $0.0698 ; 0.1810$ \\
\hline GOF & 1.042 \\
\hline \begin{tabular}{|c|} 
largest diff \\
peak and hole
\end{tabular} & 0.82 and -0.75 \\
\hline
\end{tabular}

${ }^{\text {a }} R_{1}=\Sigma\left(|| F_{\mathrm{o}}|-| F_{c}||\right) / \Sigma\left|F_{o}\right|$

${ }^{b} w R_{2}=\left\{\Sigma\left[w\left(F_{o}^{2}-F_{c}{ }^{2}\right)^{2}\right] / \Sigma\left[w\left(F_{o}{ }^{2}\right)^{2}\right]\right\}^{1 / 2}$ 
3

72

scf done: -1886.819075

$\begin{array}{llll}\mathrm{H} & -2.143641 & 2.318587 & -1.858290\end{array}$

$\begin{array}{llll}\mathrm{H} & 0.002175 & 3.276748 & -2.675048\end{array}$

$\begin{array}{llll}\mathrm{H} & 2.146402 & 2.343737 & -1.826422\end{array}$

$\begin{array}{llll}H & -1.091647 & -2.087445 & 3.684324\end{array}$

H $\quad-3.351205 \quad-0.745157 \quad 4.504068$

$\begin{array}{llll}\mathrm{H} & -4.179541 & 0.541013 & 2.226583\end{array}$

H $\quad-1.141549-3.953786-1.808111$

H $\quad-3.206552 \quad-3.265016 \quad-3.487261$

H $\quad-4.075865 \quad-0.868316 \quad-2.461787$

H $\quad-5.228270 \quad-0.780141 \quad-0.489681$

$\begin{array}{llll}\mathrm{H} & -7.323623 & 0.477011 & -0.450723\end{array}$

$\begin{array}{llll}\mathrm{H} & -5.137925 & 4.102217 & 0.235082\end{array}$

$\begin{array}{llll}\mathrm{H} & -3.038684 & 2.849555 & 0.176589\end{array}$

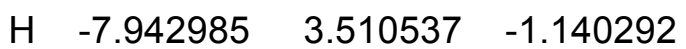

$\begin{array}{llll}\mathrm{H} & -7.614744 & 4.059004 & 0.504431\end{array}$

$\begin{array}{llll}\mathrm{H} & -8.518241 & 2.559522 & 0.230077\end{array}$

$\begin{array}{llll}H & 1.193266 & -2.270188 & 3.599700\end{array}$

H $3.452195 \quad-0.935282 \quad 4.437346$

$\begin{array}{llll}\mathrm{H} & 4.212857 & 0.472395 & 2.208784\end{array}$

H $\quad 1.008899-3.816666 \quad-2.006609$

H $3.077605 \quad-3.104106 \quad-3.667458$

H $\quad 4.045004 \quad-0.804860 \quad-2.514289$

H $\quad 5.223091 \quad-0.777453 \quad-0.556036$

$\begin{array}{llll}\mathrm{H} & 7.322651 & 0.474400 & -0.501252\end{array}$

$\begin{array}{llll}\mathrm{H} & 5.151271 & 4.083628 & 0.304965\end{array}$

$\begin{array}{llll}\mathrm{H} & 3.047761 & 2.837157 & 0.228480\end{array}$

$\begin{array}{llll}\mathrm{H} & 8.530867 & 2.528863 & 0.211892\end{array}$

$\begin{array}{llll}\mathrm{H} & 7.635373 & 4.014751 & 0.572441\end{array}$

H $\quad 7.932904 \quad 3.544063 \quad-1.101796$

$\begin{array}{llll}\text { B } & -2.557478 & 0.089532 & -0.133020\end{array}$

$\begin{array}{llll}\text { B } & 2.560370 & 0.087929 & -0.143599\end{array}$

C $\quad-1.189908 \quad 0.864333 \quad-0.591326$

C $\quad-1.198208 \quad 1.932027 \quad-1.494512$

C $\quad 0.001500 \quad 2.464827 \quad-1.951294$

$\begin{array}{llll}\text { C } & 1.200258 & 1.945879 & -1.476889\end{array}$

$\begin{array}{llll}\text { C } & 1.191086 & 0.872348 & -0.580268\end{array}$

C $\begin{array}{llll}-1.750801 & -1.447331 & 3.115862\end{array}$

$\begin{array}{llll}\text { C } & -2.898381 & -0.763568 & 3.523761\end{array}$

$\begin{array}{llll}\text { C } & -3.330710 & -0.106108 & 2.381821\end{array}$

$\begin{array}{llll}\text { C } & -1.774723 & -3.078906 & -1.783959\end{array}$

C $\quad-2.828708 \quad-2.725890 \quad-2.631076$

$\begin{array}{llll}\text { C } & -3.286699 & -1.520311 & -2.120357\end{array}$

C $\quad-3.932259 \quad 0.931601 \quad-0.250226$

$\begin{array}{llll}\text { C } & -5.181911 & 0.300772 & -0.382691\end{array}$

$\begin{array}{llll}\text { C } & -6.380795 & 1.009543 & -0.339914\end{array}$

C $\quad-6.393898 \quad 2.394141 \quad-0.139062$

$\begin{array}{llll}\text { C } & -5.162130 & 3.031111 & 0.043089\end{array}$

C $\quad-3.967263 \quad 2.314901 \quad-0.006562$

$\begin{array}{llll}\text { C } & -7.683345 & 3.169399 & -0.129871\end{array}$ 


\begin{tabular}{|c|c|c|c|}
\hline C & 1.828737 & -1.588659 & 3.052138 \\
\hline C & 2.976447 & -0.910214 & 3.468130 \\
\hline C & 3.374417 & -0.191060 & 2.350965 \\
\hline C & 1.677942 & -2.972579 & -1.932543 \\
\hline C & 2.732823 & -2.606079 & -2.773243 \\
\hline C & 3.241172 & -1.451475 & -2.197707 \\
\hline C & 3.934724 & 0.929824 & -0.255134 \\
\hline C & 5.181155 & 0.300199 & -0.418058 \\
\hline C & 6.381961 & 1.005218 & -0.366651 \\
\hline C & 6.399823 & 2.383440 & -0.126085 \\
\hline C & 5.171109 & 3.018100 & 0.083643 \\
\hline C & 3.974207 & 2.306090 & 0.024482 \\
\hline C & 7.690899 & 3.155809 & -0.104104 \\
\hline $\mathrm{N}$ & 0.000719 & 0.343651 & -0.205075 \\
\hline $\mathrm{N}$ & -2.485443 & -0.393326 & 1.369508 \\
\hline $\mathrm{N}$ & -1.511587 & -1.215507 & 1.822202 \\
\hline $\mathrm{N}$ & -2.557572 & -1.208914 & -1.030248 \\
\hline $\mathrm{N}$ & -1.604592 & -2.151001 & -0.836710 \\
\hline $\mathrm{N}$ & 2.509967 & -0.439067 & 1.344531 \\
\hline $\mathrm{N}$ & 1.557021 & -1.294698 & 1.777516 \\
\hline $\mathrm{N}$ & 2.539551 & -1.182903 & -1.078161 \\
\hline $\mathrm{N}$ & 1.556230 & -2.101968 & -0.925627 \\
\hline Co & -0.000870 & -1.621121 & 0.488284 \\
\hline
\end{tabular}

\section{2-Me}

76

scf done: -1926.703864

$\begin{array}{rrrr}\mathrm{H} & -2.113650 & 2.665743 & -1.696078 \\ \mathrm{H} & 0.039100 & 3.686690 & -2.436232 \\ \mathrm{H} & 2.175367 & 2.650081 & -1.671164 \\ \mathrm{H} & -1.055515 & -2.465830 & 3.207255 \\ \mathrm{H} & -3.281615 & -1.250418 & 4.249728 \\ \mathrm{H} & -4.079000 & 0.441321 & 2.232293 \\ \mathrm{H} & -1.037212 & -3.710661 & -1.921205 \\ \mathrm{H} & -3.198547 & -3.118124 & -3.495627 \\ \mathrm{H} & -4.077749 & -0.715752 & -2.480771 \\ \mathrm{H} & -5.167458 & -0.635096 & -0.544248 \\ \mathrm{H} & -7.284460 & 0.568394 & -0.373358 \\ \mathrm{H} & -5.159975 & 4.184254 & 0.520735 \\ \mathrm{H} & -3.037844 & 2.987840 & 0.325229 \\ \mathrm{H} & -7.987519 & 3.623548 & -0.833344 \\ \mathrm{H} & -7.626970 & 4.069408 & 0.835601 \\ \mathrm{H} & -8.504985 & 2.570675 & 0.484577 \\ \mathrm{H} & 0.914337 & -2.275047 & 3.323641 \\ \mathrm{H} & 3.121756 & -1.047347 & 4.365611 \\ \mathrm{H} & 4.052472 & 0.473119 & 2.267932 \\ \mathrm{H} & 0.886191 & -3.552175 & -2.145550 \\ \mathrm{H} & 3.039409 & -2.927379 & -3.702020 \\ \mathrm{H} & 4.060981 & -0.672366 & -2.496125 \\ \mathrm{H} & 5.164702 & -0.721218 & -0.516564 \\ \mathrm{H} & 7.313460 & 0.429997 & -0.345179 \\ \mathrm{H} & 5.278310 & 4.105016 & 0.516021 \\ \mathrm{H} & 3.126387 & 2.960072 & 0.325872 \\ \mathrm{H} & 8.578895 & 2.408881 & 0.513074\end{array}$




$\begin{array}{lrrr}\mathrm{H} & 7.738117 & 3.935931 & 0.831463 \\ \mathrm{H} & 8.099783 & 3.452133 & -0.826713 \\ \mathrm{H} & -1.091453 & -3.774154 & 0.867128 \\ \mathrm{H} & 0.506561 & -3.653773 & 1.659630 \\ \mathrm{H} & 0.411348 & -4.051275 & -0.054754 \\ \mathrm{~B} & -2.501825 & 0.280024 & -0.193683 \\ \mathrm{~B} & 2.530965 & 0.253239 & -0.176002 \\ \mathrm{C} & -1.159687 & 1.085804 & -0.596358 \\ \mathrm{C} & -1.166380 & 2.240944 & -1.383582 \\ \mathrm{C} & 0.032082 & 2.808857 & -1.794388 \\ \mathrm{C} & 1.221615 & 2.232233 & -1.369240 \\ \mathrm{C} & 1.197960 & 1.076174 & -0.584194 \\ \mathrm{C} & -1.671017 & -1.694292 & 2.775502 \\ \mathrm{C} & -2.816468 & -1.082918 & 3.289735 \\ \mathrm{C} & -3.229785 & -0.221371 & 2.287131 \\ \mathrm{C} & -1.669848 & -2.839322 & -1.898916 \\ \mathrm{C} & -2.784273 & -2.539274 & -2.683670 \\ \mathrm{C} & -3.245951 & -1.332984 & -2.179308 \\ \mathrm{C} & -3.899584 & 1.085407 & -0.221579 \\ \mathrm{C} & -5.138810 & 0.437066 & -0.368104 \\ \mathrm{C} & -6.350785 & 1.113574 & -0.247841 \\ \mathrm{C} & -6.388475 & 2.479941 & 0.051185 \\ \mathrm{C} & -5.166512 & 3.129682 & 0.251641 \\ \mathrm{C} & -3.958264 & 2.446394 & 0.122986 \\ \mathrm{C} & -7.693545 & 3.222543 & 0.145144 \\ \mathrm{C} & 1.576209 & -1.570457 & 2.850198 \\ \mathrm{C} & 2.709013 & -0.944946 & 3.373087 \\ \mathrm{C} & 3.189719 & -0.171150 & 2.330293 \\ \mathrm{C} & 1.572263 & -2.730330 & -2.038049 \\ \mathrm{C} & 2.678250 & -2.409862 & -2.825827 \\ \mathrm{C} & 3.211723 & -1.280312 & -2.225737 \\ \mathrm{C} & 3.942603 & 1.033064 & -0.203582 \\ \mathrm{C} & 5.164981 & 0.352168 & -0.343648 \\ \mathrm{C} & 6.393248 & 0.998850 & -0.225364 \\ \mathrm{C} & 6.464182 & 2.366123 & 0.063992 \\ \mathrm{C} & 5.258616 & 3.048382 & 0.255769 \\ \mathrm{C} & 4.033684 & 2.394743 & 0.129597 \\ \mathrm{C} & 7.787588 & 3.075935 & 0.155984 \\ \mathrm{C} & -0.051762 & -3.457380 & 0.739254 \\ \mathrm{~N} & 0.014594 & 0.500510 & -0.266995 \\ \mathrm{~N} & -2.369848 & -0.320854 & 1.252247 \\ \mathrm{~N} & -1.405206 & -1.212062 & 1.558993 \\ \mathrm{~N} & -2.456446 & -0.965857 & -1.153004 \\ \mathrm{~N} & -1.460073 & -1.869214 & -1.003183 \\ \mathrm{~N} & 2.378758 & -0.335482 & 1.265631 \\ \mathrm{~N} & 1.383070 & -1.187596 & 1.584004 \\ & 2.469990 & -0.978666 & -1.144054 \\ & -0.005933 & -1.551792 & 0.284914\end{array}$

$-\mathrm{CH}_{3}$ fragment

4

scf done: $\quad-39.825672$

$\begin{array}{llll}\mathrm{H} & -1.137742 & -3.680641 & 0.849137\end{array}$ 

$\begin{array}{lll}\mathrm{H} & 0.533977 & -3.549929\end{array}$
1.692907
H $\quad 0.434915-3.972383$
$-0.133425$
C $\quad-0.056256-3.733658$
0.802709

\section{2-CH $\mathrm{SiMe}_{3}$}

88

scf done: -2335.315467

B $\quad-2.161505 \quad-1.139239$

B $\quad 2.821847 \quad-0.444096$

C $\quad 5.838416 \quad-2.751614$

C $6.964593-1.984886$

C $4.552004-2.283075$

C $\quad-0.712952-1.815804$

C $4.317601-1.033724$

C $\quad 1.821661-2.757900$

C 3.4080830 .624977

C $1.624080-1.496162$

C $-0.545465-3.075753$

C $0.729496 \quad-3.545204$

C $5.459258-0.256228$

C $\quad 2.881049 \quad 1.632955$

C $\quad-2.826639 \quad-0.506341$

C $\quad-3.466702 \quad-2.079740$

C $\quad 3.387347 \quad 0.785975$

C $\quad 2.767715 \quad 1.746653$

C $\quad 6.748421-0.719422$

C $\quad-4.754475 \quad-1.537178$

C $-1.604938 \quad 1.614811$

C $\quad 1.687201 \quad 1.983878$

C $\quad-2.383034 \quad 0.393204$

C $-1.355400 \quad 1.096197$

C $-3.412834-3.402462$

C $\quad-5.908763 \quad-2.284529$

C $\quad-5.835556 \quad-3.617289$

C $\quad 1.602668 \quad 2.062715$

C $\quad-3.049079 \quad-0.040835$

C $\quad-2.669614 \quad 1.045126$

C $8.356054-2.503380$

C $\quad-4.563946 \quad-4.156319$

C $\quad-7.078681 \quad-4.440359$

C $-0.305396 \quad 3.013724$

C $\quad-3.527581 \quad 2.658932$

C $-2.235964 \quad 5.188722$

C -2.032063

Co 0.072356

4.519439

1.064868

H $\quad 5.969091$

H 3.708922

H 2.830764

$-3.726438$

$-2.901700$

$-3.095097$

0.070882

$-3.665353$

$-4.510241$

0.755630

2.060092

$-1.251173$
0.207204

0.172490

$-0.821472$

$-0.506301$

$-0.558301$

0.475036

0.040040

1.007027

$-2.155901$

0.437307

1.054639

1.344701

0.304006

$-2.945528$

$-2.253151$

0.104346

2.451404

3.234502

0.050573

0.270083

2.521781

$-2.312761$

$-3.208668$

$-2.577543$

$-0.362844$

0.049709

$-0.371453$

2.535414

2.446861

3.220932

$-0.748256$

$-0.589705$

$-0.573424$

$-0.003134$

$-0.216591$

0.844114

$-2.120535$

0.061763

$-1.287545$

$-0.855015$

1.215433

$-2.242399$

1.306443

1.826185

0.684348

$-3.842224$

$-2.290439$ 


$\begin{array}{rrrr}\mathrm{H} & 4.305818 & 0.240283 & 2.601270 \\ \mathrm{H} & 3.111365 & 2.160877 & 4.170591 \\ \mathrm{H} & 7.601579 & -0.081853 & 0.274868 \\ \mathrm{H} & -4.862545 & -0.490320 & 0.544536 \\ \mathrm{H} & -1.046667 & 2.503711 & 2.755525 \\ \mathrm{H} & 0.981868 & 2.757561 & -2.571522 \\ \mathrm{H} & -2.751973 & 0.524529 & -4.214837 \\ \mathrm{H} & -0.744815 & 1.893156 & -2.959958 \\ \mathrm{H} & -2.450791 & -3.853890 & -0.589135 \\ \mathrm{H} & -6.883619 & -1.822110 & 0.192840 \\ \mathrm{H} & 0.849778 & 2.790411 & 2.784899 \\ \mathrm{H} & -3.818015 & -0.782174 & 2.598095 \\ \mathrm{H} & -3.104093 & 1.378924 & 4.151302 \\ \mathrm{H} & 9.057296 & -1.690808 & -0.963947 \\ \mathrm{H} & 8.384196 & -3.207493 & -1.585969 \\ \mathrm{H} & 8.735686 & -3.033540 & 0.134613 \\ \mathrm{H} & -4.472398 & -5.178486 & -0.952199 \\ \mathrm{H} & -7.366556 & -4.949471 & 0.355355 \\ \mathrm{H} & -6.931754 & -5.213507 & -1.334355 \\ \mathrm{H} & -7.927681 & -3.820313 & -0.878832 \\ \mathrm{H} & -0.037351 & 3.380357 & 0.994902 \\ \mathrm{H} & 0.437113 & 3.477922 & -0.663782 \\ \mathrm{H} & -3.629491 & 2.250509 & 0.793247 \\ \mathrm{H} & -3.527552 & 1.827457 & -0.925563 \\ \mathrm{H} & -4.420308 & 3.264846 & -0.418575 \\ \mathrm{H} & -3.162330 & 5.741320 & 0.645015 \\ \mathrm{H} & -1.404354 & 5.901931 & 0.794274 \\ \mathrm{H} & -2.291422 & 4.815088 & 1.874035 \\ \mathrm{H} & -2.131784 & 3.762152 & -2.904587 \\ \mathrm{H} & -1.125858 & 5.103118 & -2.322872 \\ \mathrm{H} & -2.887568 & 5.199035 & -2.219582 \\ \mathrm{~N} & 0.369359 & -1.034392 & 0.241556 \\ \mathrm{~N} & 2.563788 & 0.396671 & -1.128376 \\ \mathrm{~N} & -2.099279 & -0.330531 & -1.130710 \\ \mathrm{~N} & -2.261788 & -0.082377 & 1.355917 \\ \mathrm{~N} & 1.499325 & 1.218403 & -1.232946 \\ \mathrm{~N} & 1.513790 & 1.332880 & 1.417405 \\ \mathrm{~N} & -1.351732 & 0.913586 & 1.410187 \\ \mathrm{~N} & 2.635712 & 0.574666 & 1.355428 \\ \mathrm{~N} & -1.196486 & 0.649759 & -1.327928 \\ \mathrm{Si} & -2.002840 & 3.759580 & -0.383264\end{array}$

$-\mathrm{CH}_{2} \mathrm{SiMe}_{3}$ fragment

16

scf done: -448.442707

$\begin{array}{rrrr}\mathrm{C} & -0.003384 & -0.003552 & -0.005745 \\ \mathrm{H} & 0.056289 & 0.135275 & -1.091523 \\ \mathrm{H} & -0.429987 & -0.994990 & 0.182773 \\ \mathrm{H} & -0.704006 & 0.742592 & 0.386549 \\ \mathrm{Si} & 1.698894 & 0.173621 & 0.789011 \\ \mathrm{C} & 2.869667 & -1.145017 & 0.117250 \\ \mathrm{H} & 3.001707 & -1.034951 & -0.965431 \\ \mathrm{H} & 2.485551 & -2.153334 & 0.307595 \\ \mathrm{H} & 3.859636 & -1.070540 & 0.581912\end{array}$




$\begin{array}{rrrr}\mathrm{C} & 2.395408 & 1.881286 & 0.367147 \\ \mathrm{H} & 2.488966 & 1.999733 & -0.719198 \\ \mathrm{H} & 1.743402 & 2.680162 & 0.737516 \\ \mathrm{H} & 3.387903 & 2.026801 & 0.807905 \\ \mathrm{C} & 1.541815 & -0.023337 & 2.630393 \\ \mathrm{H} & 0.649318 & 0.268356 & 3.184192 \\ \mathrm{H} & 2.347481 & -0.406411 & 3.256880\end{array}$

\section{2- $\mathrm{CH}_{2} \mathrm{SiMe}_{2} \mathrm{Ph}$}

95

scf done: -2526.995456

$\begin{array}{rrrr}\text { B } & -3.043756 & -0.543557 & 0.078110 \\ \text { B } & 1.808056 & -1.890947 & 0.054292 \\ \text { C } & 0.272457 & -2.409922 & 0.010315 \\ \text { C } & -0.089860 & -3.756787 & 0.089365 \\ \text { C } & -1.431104 & -4.116222 & 0.131449 \\ \text { C } & -2.391204 & -3.117012 & 0.050709 \\ \text { C } & -2.000598 & -1.775780 & -0.012362 \\ \text { C } & -1.738043 & 0.706166 & 3.254032 \\ \text { C } & -2.989224 & 0.327147 & 3.740682 \\ \text { C } & -3.654463 & -0.165680 & 2.629571 \\ \text { C } & -1.412984 & 2.426108 & -1.290160 \\ \text { C } & -2.579691 & 2.502467 & -2.053354 \\ \text { C } & -3.294363 & 1.366159 & -1.712288 \\ \text { C } & -4.580474 & -0.832136 & -0.317416 \\ \text { C } & -5.640783 & -0.047682 & 0.170494 \\ \text { C } & -6.949345 & -0.204317 & -0.280594 \\ \text { C } & -7.264998 & -1.144840 & -1.267684 \\ \text { C } & -6.214348 & -1.898495 & -1.800050 \\ \text { C } & -4.908386 & -1.739721 & -1.338183 \\ \text { C } & -8.682886 & -1.342541 & -1.730487 \\ \text { C } & 1.416993 & -0.066912 & 3.179259 \\ \text { C } & 2.352823 & -0.983364 & 3.659099 \\ \text { C } & 2.651746 & -1.776607 & 2.561983 \\ \text { C } & 1.478818 & 1.256748 & -1.786530 \\ \text { C } & 2.466415 & 0.683234 & -2.588975 \\ \text { C } & 2.734036 & -0.538191 & -1.994165 \\ \text { C } & 2.994415 & -2.910267 & -0.338919 \\ \text { C } & 4.321579 & -2.650882 & 0.050446 \\ \text { C } & 5.389582 & -3.431450 & -0.384949 \\ \text { C } & 5.187981 & -4.507650 & -1.256817 \\ \text { C } & 3.881864 & -4.747048 & -1.694771 \\ \text { C } & 2.817507 & -3.962884 & -1.250835 \\ \text { C } & 6.333252 & -5.377680 & -1.698539 \\ \text { C } & 0.419195 & 2.280800 & 1.320954 \\ \text { C } & 2.481489 & 4.066863 & 2.637901 \\ \text { C } & 3.577665 & 1.718228 & 1.048191 \\ \text { C } & 2.241980 & 4.086514 & -0.396963 \\ \text { C } & 1.226729 & 5.030357 & -0.630937 \\ \text { C } & 1.234570 & 5.847639 & -1.761889 \\ \text { C } & 2.272082 & 5.742726 & -2.688409 \\ \text { C } & 3.299018 & 4.823984 & -2.471133 \\ \text { C } & 3.280294 & 4.009008 & -1.339182 \\ \text { Co } & -0.141276 & 0.503237 & 0.636484\end{array}$




\begin{tabular}{|c|c|c|c|}
\hline $\mathrm{H}$ & 0.684756 & -4.512745 & 0.150205 \\
\hline $\mathrm{H}$ & -1.722771 & -5.159639 & 0.224874 \\
\hline $\mathrm{H}$ & -3.446793 & -3.362853 & 0.076291 \\
\hline $\mathrm{H}$ & -0.923572 & 1.161610 & 3.789636 \\
\hline $\mathrm{H}$ & -3.357222 & 0.404834 & 4.752859 \\
\hline $\mathrm{H}$ & -4.642801 & -0.586276 & 2.529375 \\
\hline $\mathrm{H}$ & -0.594682 & 3.126024 & -1.220530 \\
\hline $\mathrm{H}$ & -2.864683 & 3.279336 & -2.747006 \\
\hline $\mathrm{H}$ & -4.264180 & 1.019783 & -2.032990 \\
\hline $\mathrm{H}$ & -5.439540 & 0.734858 & 0.897874 \\
\hline $\mathrm{H}$ & -7.737544 & 0.424340 & 0.129590 \\
\hline $\mathrm{H}$ & -6.419058 & -2.614578 & -2.593696 \\
\hline $\mathrm{H}$ & -4.121630 & -2.323564 & -1.809054 \\
\hline $\mathrm{H}$ & -9.257214 & -0.411829 & -1.679191 \\
\hline $\mathrm{H}$ & -9.201197 & -2.078005 & -1.101905 \\
\hline $\mathrm{H}$ & -8.723758 & -1.710253 & -2.760690 \\
\hline $\mathrm{H}$ & 0.958342 & 0.756260 & 3.697598 \\
\hline $\mathrm{H}$ & 2.756600 & -1.055215 & 4.657918 \\
\hline $\mathrm{H}$ & 3.312603 & -2.623334 & 2.462084 \\
\hline $\mathrm{H}$ & 1.008211 & 2.217011 & -1.884474 \\
\hline $\mathrm{H}$ & 3.423301 & -1.321890 & -2.265864 \\
\hline $\mathrm{H}$ & 4.535260 & -1.793963 & 0.685206 \\
\hline $\mathrm{H}$ & 6.398941 & -3.193441 & -0.054416 \\
\hline $\mathrm{H}$ & 3.695064 & -5.553090 & -2.401870 \\
\hline $\mathrm{H}$ & 1.829210 & -4.162984 & -1.655295 \\
\hline $\mathrm{H}$ & 7.271386 & -4.815341 & -1.746878 \\
\hline $\mathrm{H}$ & 6.149794 & -5.817295 & -2.684104 \\
\hline $\mathrm{H}$ & 6.488472 & -6.206979 & -0.996510 \\
\hline $\mathrm{H}$ & -0.299493 & 3.021465 & 0.949281 \\
\hline $\mathrm{H}$ & 0.230930 & 2.246030 & 2.400558 \\
\hline $\mathrm{H}$ & 1.699951 & 4.827757 & 2.746427 \\
\hline $\mathrm{H}$ & 3.444068 & 4.585270 & 2.556720 \\
\hline $\mathrm{H}$ & 2.498172 & 3.471853 & 3.559534 \\
\hline $\mathrm{H}$ & 3.551215 & 1.051440 & 1.915965 \\
\hline $\mathrm{H}$ & 4.539785 & 2.244415 & 1.069474 \\
\hline $\mathrm{H}$ & 3.549321 & 1.098556 & 0.148761 \\
\hline $\mathrm{H}$ & 0.406779 & 5.129145 & 0.078961 \\
\hline $\mathrm{H}$ & 0.432668 & 6.564735 & -1.920315 \\
\hline $\mathrm{H}$ & 2.280727 & 6.373914 & -3.573390 \\
\hline $\mathrm{H}$ & 4.112051 & 4.737956 & -3.188136 \\
\hline $\mathrm{H}$ & 4.077878 & 3.282932 & -1.201902 \\
\hline $\mathrm{H}$ & 2.923666 & 1.107838 & -3.469800 \\
\hline$N$ & -0.688355 & -1.454486 & 0.028692 \\
\hline$N$ & -2.843961 & -0.058364 & 1.560782 \\
\hline$N$ & -1.646097 & 0.446979 & 1.944604 \\
\hline$N$ & -2.579263 & 0.663339 & -0.809143 \\
\hline$N$ & -1.419303 & 1.304749 & -0.562239 \\
\hline$N$ & 1.944012 & -1.330445 & 1.508032 \\
\hline$N$ & 1.158872 & -0.298797 & 1.886040 \\
\hline$N$ & 1.949918 & -0.663334 & -0.903078 \\
\hline $\mathrm{N}$ & 1.179521 & 0.437119 & -0.773837 \\
\hline Si & 2.164580 & 2.962375 & 1.129259 \\
\hline
\end{tabular}


$-\mathrm{CH}_{2} \mathrm{SiMe}_{2} \mathrm{Ph}$ fragment

23

scf done: -640.118980

C $0.795575 \quad-0.862290$

13.276839

C $\quad 0.880940-1.448489$

C $\quad 2.637435 \quad 0.797538$

C $\quad-0.425521 \quad 1.142930$

16.266108

15.120481

C $\quad-1.724131 \quad 0.825407$

15.195995

C -2.787700

1.705730

14.762552

C -2.572515

2.931908

14.952260

C -1.292044

3.270447

15.581466

C -0.232630

2.383881

16.016279

H 0.416198

$-0.313641$

15.823332

H 1.095914

$-1.887042$

12.415265

H $\quad-0.085385-1.963057$

13.058358

H $0.999093 \quad-1.028339$

16.230183

H $1.668157 \quad-2.197393$

17.271238

H 3.463915

0.106588

16.119383

$\begin{array}{ll}\mathrm{H} & 2.776587\end{array}$

1.191788

14.920273

H 2.718921

1.631639

16.133943

H -1.906281

$-0.124149$

14.415533

$\begin{array}{ll}\mathrm{H} & -3.783337\end{array}$

1.438397

14.261697

14.607277

H $\quad-3.399287$

3.621809

15.729466

H -1.117955

4.226670

16.503231

H $\quad 0.759499 \quad 2.670303$

16.165364

Si $\quad 0.981551-0.094632$

14.957480

\section{2-'Bu}

85

scf done: -2044.623394

$\begin{array}{lrrr}\text { B } & -2.617304 & -0.499030 & 0.185235 \\ \text { B } & 2.410776 & -0.726308 & 0.198101 \\ \text { C } & -1.326197 & -1.431763 & 0.459380 \\ \text { C } & -1.402980 & -2.693832 & 1.057083 \\ \text { C } & -0.239897 & -3.367238 & 1.404995 \\ \text { C } & 0.984480 & -2.786895 & 1.097828 \\ \text { C } & 1.030736 & -1.530058 & 0.488919 \\ \text { C } & -1.743420 & 2.017999 & -2.314136 \\ \text { C } & -2.909623 & 1.561644 & -2.932781 \\ \text { C } & -3.328385 & 0.502237 & -2.144607 \\ \text { C } & -1.642295 & 2.187542 & 2.468616 \\ \text { C } & -2.772812 & 1.784884 & 3.180162 \\ \text { C } & -3.291294 & 0.736995 & 2.436111 \\ \text { C } & -4.052710 & -1.226941 & 0.073062 \\ \text { C } & -5.261546 & -0.557123 & 0.332268 \\ \text { C } & -6.502382 & -1.144388 & 0.094734 \\ \text { C } & -6.600450 & -2.433419 & -0.440645 \\ \text { C } & -5.408164 & -3.094925 & -0.751625 \\ \text { C } & -4.170764 & -2.502343 & -0.504492 \\ \text { C } & -7.937280 & -3.086512 & -0.664104 \\ \text { C } & 1.321134 & 1.253389 & -2.677349 \\ \text { C } & 2.406234 & 0.626882 & -3.291051 \\ \text { C } & 2.979538 & -0.134478 & -2.287522\end{array}$




\begin{tabular}{|c|c|c|c|}
\hline C & 1.603595 & 1.977846 & 2.489364 \\
\hline C & 2.710178 & 1.506958 & 3.195773 \\
\hline C & 3.193998 & 0.464851 & 2.421552 \\
\hline C & 3.789597 & -1.558801 & 0.104068 \\
\hline C & 5.033305 & -0.922608 & 0.270353 \\
\hline C & 6.240590 & -1.582312 & 0.053028 \\
\hline C & 6.267754 & -2.917667 & -0.365141 \\
\hline C & 5.040033 & -3.550725 & -0.581962 \\
\hline C & 3.835949 & -2.883926 & -0.358295 \\
\hline C & 7.569660 & -3.644700 & -0.565559 \\
\hline C & 0.064824 & 3.243386 & -0.043848 \\
\hline C & 1.121757 & 3.965319 & -0.874851 \\
\hline C & 0.842709 & 5.476046 & -0.851139 \\
\hline C & 2.549504 & 3.688740 & -0.403498 \\
\hline Co & -0.030498 & 1.261572 & 0.026565 \\
\hline $\mathrm{H}$ & -2.374906 & -3.120044 & 1.279201 \\
\hline $\mathrm{H}$ & -0.285972 & -4.330313 & 1.907942 \\
\hline $\mathrm{H}$ & 1.910748 & -3.287068 & 1.357171 \\
\hline $\mathrm{H}$ & -1.111524 & 2.855532 & -2.567671 \\
\hline $\mathrm{H}$ & -3.385649 & 1.953426 & -3.819293 \\
\hline $\mathrm{H}$ & -4.196019 & -0.133772 & -2.222173 \\
\hline $\mathrm{H}$ & -0.969010 & 3.000011 & 2.681975 \\
\hline $\mathrm{H}$ & -3.159393 & 2.199377 & 4.099244 \\
\hline $\mathrm{H}$ & -4.151772 & 0.109622 & 2.608415 \\
\hline $\mathrm{H}$ & -5.241250 & 0.467185 & 0.695593 \\
\hline $\mathrm{H}$ & -7.410940 & -0.586973 & 0.315005 \\
\hline $\mathrm{H}$ & -5.448186 & -4.085237 & -1.201351 \\
\hline $\mathrm{H}$ & -3.274009 & -3.043027 & -0.796081 \\
\hline $\mathrm{H}$ & -8.246037 & -3.659327 & 0.219764 \\
\hline $\mathrm{H}$ & -7.909865 & -3.783471 & -1.507905 \\
\hline $\mathrm{H}$ & -8.720116 & -2.346789 & -0.860138 \\
\hline $\mathrm{H}$ & 0.601067 & 1.925003 & -3.108589 \\
\hline $\mathrm{H}$ & 2.724953 & 0.715588 & -4.318894 \\
\hline $\mathrm{H}$ & 3.834628 & -0.791551 & -2.299955 \\
\hline $\mathrm{H}$ & 0.958798 & 2.803253 & 2.732688 \\
\hline $\mathrm{H}$ & 3.102483 & 1.874400 & 4.132247 \\
\hline $\mathrm{H}$ & 4.024119 & -0.205951 & 2.578969 \\
\hline $\mathrm{H}$ & 5.064242 & 0.128809 & 0.546228 \\
\hline $\mathrm{H}$ & 7.178148 & -1.048320 & 0.196593 \\
\hline $\mathrm{H}$ & 5.025014 & -4.578097 & -0.940955 \\
\hline $\mathrm{H}$ & 2.910376 & -3.406576 & -0.583669 \\
\hline $\mathrm{H}$ & 8.359337 & -2.969412 & -0.910843 \\
\hline $\mathrm{H}$ & 7.471722 & -4.453969 & -1.296085 \\
\hline $\mathrm{H}$ & 7.916230 & -4.094437 & 0.373739 \\
\hline $\mathrm{H}$ & -0.939968 & 3.566903 & -0.340320 \\
\hline $\mathrm{H}$ & 0.193888 & 3.560649 & 0.997354 \\
\hline $\mathrm{H}$ & 1.047464 & 3.644252 & -1.921078 \\
\hline $\mathrm{H}$ & 0.903252 & 5.867467 & 0.172504 \\
\hline $\mathrm{H}$ & 1.571387 & 6.026299 & -1.459552 \\
\hline $\mathrm{H}$ & -0.157959 & 5.707470 & -1.234511 \\
\hline $\mathrm{H}$ & 3.279399 & 4.223621 & -1.022546 \\
\hline $\mathrm{H}$ & 2.684155 & 4.020632 & 0.633377 \\
\hline $\mathrm{H}$ & 2.786741 & 2.623522 & -0.438723 \\
\hline $\mathrm{N}$ & -0.120979 & -0.860148 & 0.245099 \\
\hline $\mathrm{N}$ & -2.450097 & 0.344350 & -1.131721 \\
\hline
\end{tabular}




$\begin{array}{lrrr}N & -1.469329 & 1.261356 & -1.247971 \\ N & -2.518765 & 0.557363 & 1.348943 \\ N & -1.476528 & 1.423922 & 1.382838 \\ N & 2.268931 & 0.044416 & -1.155177 \\ N & 1.252332 & 0.902208 & -1.388770 \\ N & 2.422436 & 0.354922 & 1.324244 \\ N & 1.423177 & 1.264635 & 1.371120\end{array}$

$-\mathrm{CH}_{2}{ }^{\mathrm{i}} \mathrm{Pr}$ fragment

13

scf done: -157.752819

$\begin{array}{rrrr}\mathrm{C} & 0.138181 & -1.408402 & 0.176199 \\ \mathrm{C} & 0.714233 & -0.593539 & 1.285127 \\ \mathrm{C} & 2.247225 & -0.616485 & 1.261279 \\ \mathrm{C} & 0.181696 & 0.844169 & 1.260939 \\ \mathrm{H} & 0.648199 & -2.298172 & -0.182938 \\ \mathrm{H} & -0.870630 & -1.224149 & -0.183160 \\ \mathrm{H} & 0.397118 & -1.041758 & 2.246707 \\ \mathrm{H} & 2.664318 & -0.060045 & 2.107860 \\ \mathrm{H} & 2.631807 & -1.641282 & 1.311541 \\ \mathrm{H} & 2.626056 & -0.161827 & 0.338532 \\ \mathrm{H} & -0.912697 & 0.865230 & 1.310931 \\ \mathrm{H} & 0.567107 & 1.422990 & 2.107530 \\ \mathrm{H} & 0.484332 & 1.352736 & 0.338193\end{array}$

